\title{
NTT and NOT spectroscopy of SDSS-II supernovae
}

L. Östman ${ }^{1,2,3}$, J. Nordin ${ }^{1,3}$, A. Goobar ${ }^{1,3}$, R. Amanullah ${ }^{1,3}$, M. Smith ${ }^{4,5}$, J. Sollerman ${ }^{3,6,7}$, V. Stanishev ${ }^{8}$, M. D. Stritzinger ${ }^{3,7,9}$, B. A. Bassett ${ }^{5,10,11}$, T. M. Davis ${ }^{7,12}$, E. Edmondson ${ }^{4}$, J. A. Frieman ${ }^{13,14}$, P. M. Garnavich ${ }^{15}$, H. Lampeitl ${ }^{4}$, G. Leloudas ${ }^{7}$, J. Marriner ${ }^{13}$, R. C. Nichol $^{4}$, K. Romer ${ }^{16}$, M. Sako ${ }^{17}$, D. P. Schneider ${ }^{18}$, and C. Zheng ${ }^{19}$

${ }^{1}$ Department of Physics, Stockholm University, 10691 Stockholm, Sweden

e-mail: linda@ifae.es

2 Institut de Física d'Altes Energies, 08193 Bellaterra, Barcelona, Spain

3 Oskar Klein Centre for Cosmo Particle Physics, AlbaNova University Center, 10691 Stockholm, Sweden

Institute of Cosmology and Gravitation, Portsmouth PO13FX, UK

Department of Mathematics and Applied Mathematics, University of Cape Town, South Africa

Astronomy Department, Stockholm University, 10691 Stockholm, Sweden

Dark Cosmology Centre, Niels Bohr Institute, University of Copenhagen, 2100 Copenhagen $\emptyset$, Denmark

8 CENTRA - Centro Multidisciplinar de Astrofísica, Instituto Superior Técnico, 1049-001 Lisbon, Portugal

9 Carnegie Institute for Science, Carnegie Observatories, Casilla 601, La Serena, Chile

10 South African Astronomical Observatory, Cape Town, South Africa

11 African Institute for Mathematical Sciences, Muizenberg, Cape Town, South Africa

12 School of Mathematics and Physics, University of Queensland, QLD 4072, Australia

13 Center for Particle Astrophysics, Fermi National Accelerator Laboratory, Batavia, Illinois 60510, USA

14 Kavli Institute for Cosmological Physics, University of Chicago, Chicago, Illinois 60637, USA

15 University of Notre Dame, Notre Dame, IN 46556-5670, USA

16 Department of Physics and Astronomy, University of Sussex, UK

17 Department of Physics and Astronomy, University of Pennsylvania, Philadelphia, PA 19104, USA

18 Department of Astronomy and Astrophysics, Pennsylvania State University, University Park, PA 16802 USA

19 Kavli Institute for Particle Astrophysics and Cosmology, Stanford University, Stanford, CA 94305-4060, USA

Received 7 September 2010 / Accepted 16 October 2010

\section{ABSTRACT}

Context. The Sloan Digital Sky Survey II (SDSS-II) Supernova Survey, conducted between 2005 and 2007, was designed to detect a large number of type Ia supernovae around $z \sim 0.2$, the redshift "gap" between low- $z$ and high- $z$ supernova searches. The survey has provided multi-band (ugriz) photometric lightcurves for variable targets, and supernova candidates were scheduled for spectroscopic observations, primarily to provide supernova classification and accurate redshifts. We present supernova spectra obtained in 2006 and 2007 using the New Technology Telescope (NTT) and the Nordic Optical Telescope (NOT).

Aims. We provide an atlas of supernova spectra in the range $z=0.03-0.32$ that complements the well-sampled lightcurves from SDSS-II in the forthcoming three-year SDSS supernova cosmology analysis. The sample can, for example, be used for spectral studies of type Ia supernovae, which are critical for understanding potential systematic effects when supernovae are used to determine cosmological distances.

Methods. The spectra were reduced in a uniform manner, and special care was taken in estimating the uncertainties for the different processing steps. Host-galaxy light was subtracted when possible and the supernova type fitted using the SuperNova IDentification code (SNID). We also present comparisons between spectral and photometric dating using SALT lightcurve fits to the photometry from SDSS-II, as well as the global distribution of our sample in terms of the lightcurve parameters: stretch and colour.

Results. We report new spectroscopic data from 141 type Ia supernovae, mainly between -9 and +15 days from lightcurve maximum, including a few cases of multi-epoch observations. This homogeneous, host-galaxy subtracted, type Ia supernova spectroscopic sample is among the largest such data sets and unique in its redshift interval. The sample includes two potential SN 1991T-like supernovae (SN 2006on and SN 2007ni) and one potential SN 2002cx-like supernova (SN 2007ie). In addition, the new compilation includes spectra from 23 confirmed type II and 8 type Ib/c supernovae.

Key words. methods: observational - techniques: spectroscopic - supernovae: general - surveys - cosmology: observations

\section{Introduction}

Type Ia supernovae ( $\mathrm{SNe}$ Ia) as distance indicators provided the first direct evidence of the late-time acceleration of the Universe (Perlmutter et al. 1999; Riess et al. 1998). The observed faintness of high- $z$ SNe Ia suggests there is an energy component with a negative pressure, which has been given the name dark energy. The origin and nature of the dark energy is still unknown, and precise measurements of its equation of state remain as one of the key goals for both cosmology and fundamental physics. To achieve the necessary precision using $\mathrm{SNe} \mathrm{Ia}$, large statistical samples and excellent control of potential systematic effects are imperative.

The Sloan Digital Sky Survey II (SDSS-II) Supernova Survey (York et al. 2000; Frieman et al. 2008) operated as a three-year (3 months per campaign) program (2005-2007), aimed at detecting a significant number of intermediate-redshift $\mathrm{SNe}$ Ia in a rolling survey. The survey provided multi-band 
(ugriz) photometric lightcurves for transient targets. The SN candidates detected in the galaxy subtraction pipeline were scheduled for spectroscopic observation, in order to provide spectral identification and an accurate redshift. SN Ia candidates were given highest priority for spectroscopic follow-up, however other SN types were also observed.

The first-year photometry and spectroscopy have been presented in Holtzman et al. (2008) and Zheng et al. (2008), respectively. First cosmological results, including a Hubble diagram consisting of $103 \mathrm{SNe}$ Ia discovered during the first year of the survey can be found in Kessler et al. (2009) (see also Sollerman et al. 2009; Lampeitl et al. 2010).

In this article we present optical spectroscopy obtained with the ESO New Technology Telescope (NTT) and the Nordic Optical Telescope (NOT). In total, 290 spectra of SDSS-II SN candidates were obtained during 2006 and 2007. The data set contains 169 confirmed SN Ia spectra from 141 objects in the redshift range $0.03<z<0.32$. The redshift range complements previous and current SN Ia surveys and the spectra are, in general, of high signal-to-noise ratio $(\mathrm{S} / \mathrm{N})$.

The NTT and the NOT spectra constitute a natural subset of the complete SDSS sample since many of the observers were active at both telescopes guaranteeing a consistent observing strategy as well as similar choices of targets. Furthermore, all data were processed using the same pipeline, in a coherent manner including a detailed error analysis. The reduction procedure is described in this paper. The data set is presented as a documented library available for future studies of SNe Ia.

When our SN Ia spectra are combined with low-redshift spectroscopic samples, they cover a wide interval in cosmic time. They can then be used to search for signs of evolution of luminosity with distance, a potentially large systematic uncertainty in the use of SNe Ia as distance indicators (see e.g., Nordin et al. 2008). A statistical study of spectral features of $\mathrm{SNe}$ Ia could provide evidence for or against such evolution. In order to make such a study possible, correct estimates of the uncertainties are essential, especially if comparisons are to be made to a high-S/N local sample. In a companion paper, Nordin et al. (2011) present initial results of such a quantitative study. Similar studies, using data sets in other redshift ranges have previously been made (Hook et al. 2005; Blondin et al. 2006; Garavini et al. 2007; Foley et al. 2008; Bronder et al. 2008). Furthermore, spectroscopic features can potentially be used as brightness indicators (Bronder et al. 2008). Spectroscopic studies of SNe also play an important role to constrain the progenitor systems, and the explosion physics.

Other large spectroscopic samples which have been made accessible to the community include the low-redshift sample of Matheson et al. (2008) (432 spectra of 32 SNe Ia) and the highredshift sample of Balland et al. (2009) (139 ESO/VLT spectra of $124 \mathrm{SNe}$ Ia from SNLS with an average redshift of 0.63). The NTT/NOT data set complements these two well, having a mean redshift of 0.16 and a similar number of spectra as the SNLS spectra. The $\mathrm{S} / \mathrm{N}$ of our data set is lower than the lowredshift sample and higher than the SNLS sample. Descriptions of other medium- and high-redshift samples have been presented in e.g. Howell et al. (2005); Foley et al. (2009); Zheng et al. (2008).

The NTT/NOT sample also contains spectra of core-collapse $\mathrm{SNe}$. Some of the SNe IIP presented in this paper were used by D'Andrea et al. (2010) to investigate the method where luminosity is standardised using the ejecta velocity during plateau phase.

The paper is organised in the following manner. Section 2 describes the spectroscopic observations performed with the NTT and the NOT. In Sect. 3 the data set is presented and comparisons with other data sets are made. Section 4 describes the reduction method. The problems with differential atmospheric refraction and slit losses are addressed in Sect. 5. Section 6 deals with the host-galaxy subtraction and Sect. 7 with the typing. A couple of specific objects are briefly discussed in Sect. 8. This is followed by a summary in Sect. 9. The Appendix contains three tables describing the spectra: the observations (A.1), the typing and redshifts (A.2) and the quality of the data (A.3).

\section{Observations}

The NTT, located at the La Silla Observatory in Chile, was used for spectroscopic observations of SDSS-II SN candidates from September to December in 2006 and 2007 ${ }^{1}$. Thirty-four nights were awarded for the project, out of which 32 had sufficiently good conditions to obtain SN spectra. Through the course of these nights, 244 spectra of SN candidates were obtained. The primary mirror of the NTT has a diameter of $3.58-\mathrm{m}$. The observations were performed using the ESO Multi-Mode Instrument (EMMI; Dekker et al. 1986) in the Red Imaging and Low-Dispersion spectroscopy (RILD) mode using grism 2. Grism 2 provides a wavelength coverage from 3800 to $9200 \AA$. It has 300 grooves per mm, a wavelength dispersion of $1.74 \AA$ per pixel, and a spatial resolution of 0 ' 166 per pixel, without CCD binning. During the observations a binning of $2 \times 2$ was used, resulting in a resolving power $R \simeq 570$ at $6000 \AA$ for a 1 .'0 slit. Slit widths of 1 .' 0 or $1^{\prime \prime} .5$ were used depending on the seeing conditions.

The NOT is located at the Observatorio del Roque de los Muchachos on La Palma, Spain. Spectroscopic observations were conducted with this telescope during November 2006 and September and November $2007^{2}$. Eleven nights were awarded for the project, out of which 9 nights had good enough observing conditions to obtain SN spectroscopy. During these nights 46 spectra of target $\mathrm{SNe}$ were obtained. The primary mirror of the NOT has a diameter of 2.56-m. Spectra were obtained with the Andalucia Faint Object Spectrograph and Camera (ALFOSC) using grism 4, which has 300 grooves per mm. This set-up provides a wavelength range from 3200 to $9100 \AA$ with a wavelength dispersion of $\sim 3.0 \AA$ per pixel and a spatial resolution of $0^{\prime} .19$ per pixel. The resolving power is 710 for a $0 .{ }^{\prime} 5$ slit. Depending on the seeing, slit widths of $11^{\prime \prime} 0$ or 1 1.'3 were used.

In general, to avoid observing erroneous objects such as asteroids and AGNs, each candidate had at least two epochs of photometry before they were placed in our spectroscopic queue. High priority was given to probable SNe Ia which appeared to have been discovered while still being on the rise. Furthermore, since several telescopes were taking spectra on the same nights, a division was made where faint targets were typically allocated to telescopes with a larger aperture. More details about the procedure for selecting the objects for spectroscopic observation can be found in Sako et al. (2008).

A typical exposure time for the spectroscopic observations was $1800 \mathrm{~s}$, but depending on the magnitude of the SN and the observing conditions, exposure times were varied between 300 and $3600 \mathrm{~s}$. In Table A.1 the exposure time for each individual spectrum is given.

\footnotetext{
1 The observations were acquired in the ESO programmes 077.A0437, 078.A-0325, 079.A-0715 and 080.A-0024 under PI Robert Nichol.

2 The observations were acquired in the programmes with proposal numbers 34-004, 35-023 and 36-010 under PI Maximilian Stritzinger.
} 


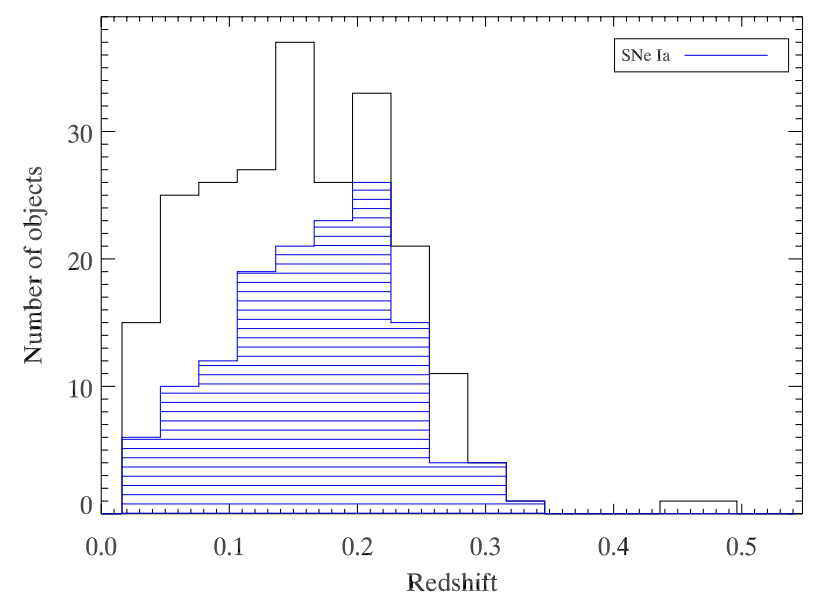

Fig. 1. Redshift distribution of the 228 observed objects at the NTT and the NOT with a known redshift. The subset of objects classified as $\mathrm{SNe}$ Ia is shown in the striped histogram. The bin size in the plot is 0.03 .

As part of the observing programme, host-galaxy spectra of previously confirmed SNe Ia were obtained when SN candidates were lacking or the observational conditions were poor. These host galaxy spectra will not be presented in this paper.

\section{Data set}

The data set contains 290 spectra of 238 individual objects. Information about the spectra are given in the tables in the Appendix. Each object has been given a unique SDSS ID and the spectra have been given unique spectral numbers, SPIDs. Redshifts were obtained from Zheng et al. (in prep.). Out of the 238 targets, 84 had prior galaxy redshifts from SDSS DR7, 102 objects had the redshift determined from galaxy lines and 40 from SN features. Some of these redshifts were determined from NTT/NOT spectra, but also observations from other telescopes were used. Furthermore, there were two objects (ID 16391 and 16838) for which no reliable redshifts could be obtained from either the host galaxy or SN features, but where a redshift could be determined through a rough template fitting. For 10 objects, no redshift could be determined at all. Most of these objects are seemingly "hostless" events, several exhibiting lightcurves which differ significantly from that of SNe. The redshifts and the origin of the redshifts are listed in Table A.2. The redshift distribution of the objects is shown in Fig. 1, where the subset of objects which were classified as SNe Ia is indicated. The redshift range for all objects observed was $z=0.016-0.487$, and for the subset of SNe Ia, $z_{\text {SNIa }}=0.031-0.324$.

Our SN Ia sample contains 169 spectra of 141 individual objects, plus 3 likely SNe Ia. In addition, we also obtained 26 spectra of $23 \mathrm{SNe}$ II and 12 spectra of $8 \mathrm{SNe} \mathrm{Ib/c.} \mathrm{Fourteen} \mathrm{spectra}$ were identified as galaxy spectra, which presumably were the result of poor seeing conditions or that the spectra were taken when the SN had faded significantly, becoming much fainter than the host galaxy. These spectra are useful as they provide spectroscopic redshift determination for the photometric sample of SNe. In Sect. 7 we describe how the spectroscopic typing was performed. The number of spectra and the number of unique objects are summarised in Table 1. For the first four years of ESSENCE, $55 \%$ of the spectroscopically observed objects were identified as $\mathrm{SNe}$ Ia, probable SNe Ia or core collapse supernovae (Foley et al. 2009). The corresponding number for the NTT/NOT data set is $72 \%$. The SN fraction obtained depends on the design of
Table 1. Number of spectra and unique objects.

\begin{tabular}{lll}
\hline \hline & Spectra & Objects \\
\hline Total & 290 & 238 \\
with redshift & 280 & 228 \\
\hline SN Ia & 169 & 141 \\
with good LC & 127 & 108 \\
SN Ia? & 3 & 3 \\
SN II & 26 & 23 \\
SN Ib/c & 12 & 8 \\
Not SN & 19 & 16 \\
Galaxy & 14 & 12 \\
Unclassified & 61 & 47 \\
\hline
\end{tabular}

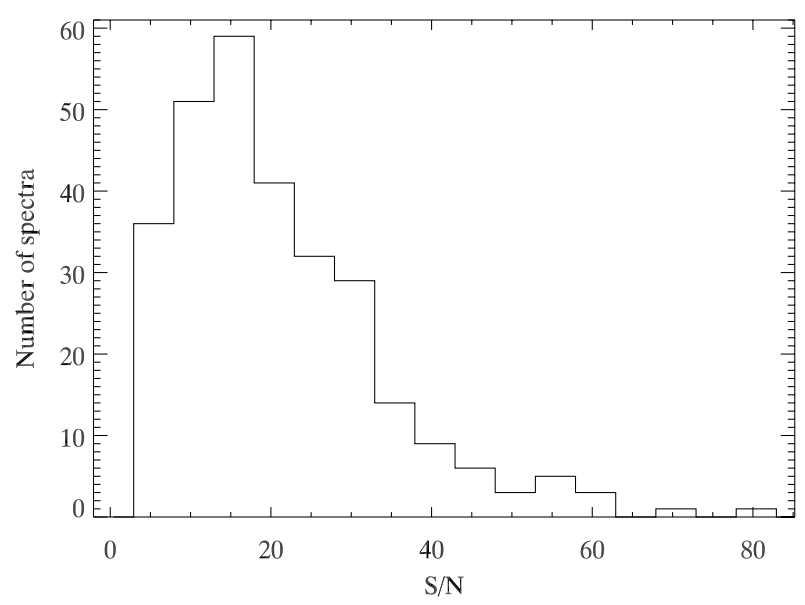

Fig. 2. Distribution of the $\mathrm{S} / \mathrm{N}$ for the spectra taken at the NTT and the NOT. The $\mathrm{S} / \mathrm{N}$ is calculated in $10 \AA$ bins and averaged over the interval between 4000 and $6500 \AA$ (in observed frame).

the search, the redshift interval, the size of the telescope and the time spent on each supernova target. As two of several telescopes (with different sizes) involved in the follow-up of SDSS SNe, where different targets were assigned to different telescopes, the evaluation of the efficiency is further complicated.

The spectroscopic S/N distribution for the 290 NTT and NOT spectra is shown in Fig. 2. Values are computed for $10 \AA$ bins and then averaged over the interval between 4000 and $6500 \AA$ (in observed frame), which is the interval of most interest for $\mathrm{SN}$ features.

\subsection{Lightcurve properties}

SDSS multi-band photometry of the objects in our sample and their host galaxies were obtained from ugriz (Fukugita et al. 1996) observations using the method presented in Holtzman et al. (2008). Lightcurves of all confirmed SNe Ia were fitted using SALT (Guy et al. 2005). The same authors have since published a new fitter, SALT2 (Guy et al. 2007). Even though SALT2 in many respects is an improvement, we use the older code since it provides smooth lightcurves which are easy to use for interpolation, e.g. for calculating the photometry of the spectral epoch, to estimate the host galaxy contamination. The fitted parameters are: lightcurve width $s$ ("stretch"), SALT colour $c$, rest-frame peak $B$-band magnitude, and time of $B$-band maximum light. However, 33 SN Ia lightcurves lack either pre- or post-maximum photometry. This leads to bigger uncertainties for the derived lightcurve parameters. In the remainder of this paper, we will refer to these as poor lightcurves. Removing all 


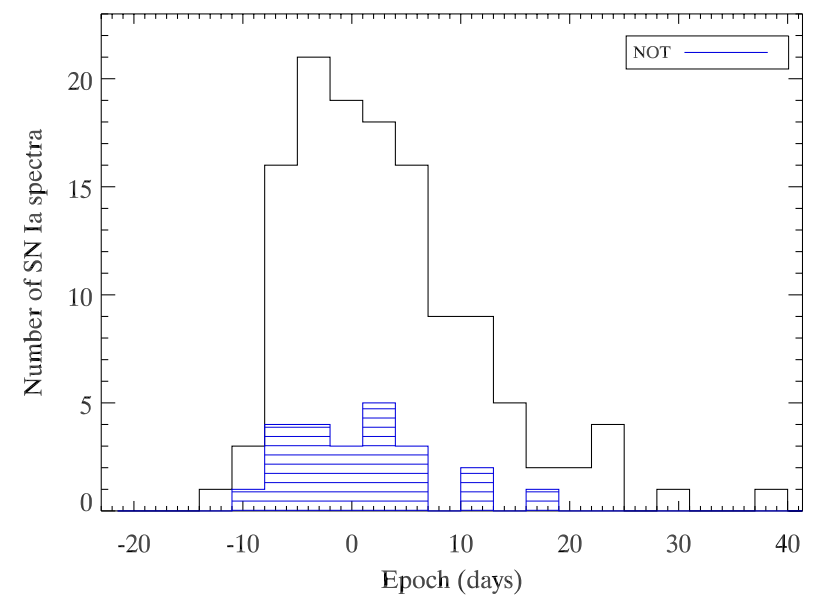

Fig. 3. Epoch distribution of the SN Ia spectra observed at the NTT and the NOT, excluding the SNe with poor lightcurves. The un-filled histogram shows the full sample of SN Ia spectra, while the striped histogram shows the subset observed at the NOT. The epoch is defined as the number of days in rest frame since $B$-band maximum brightness, as obtained from the lightcurve. The 127 spectra are divided into epoch bins with a width of 3 days.

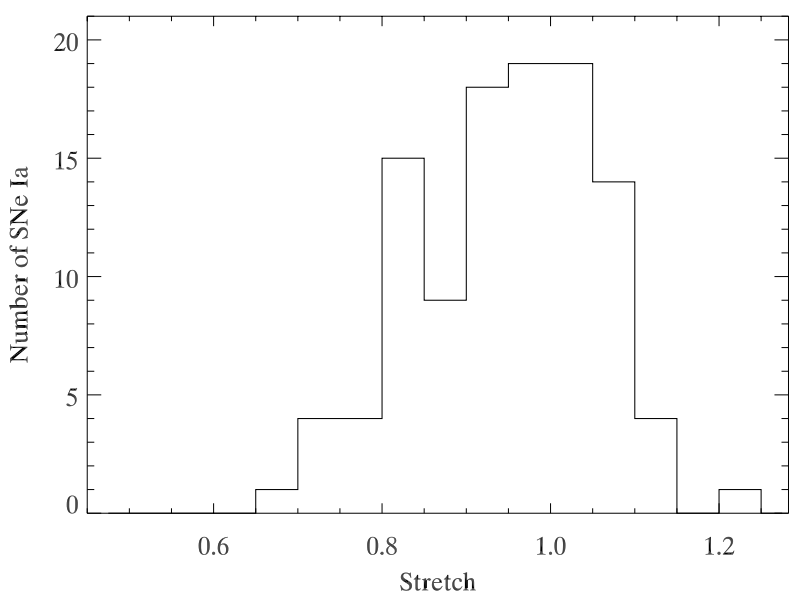

Fig. 4. Distribution of stretch (SALT $s$ parameter) of the $\mathrm{SNe} \mathrm{Ia}$ observed at the NTT and the NOT, excluding the SNe with poor lightcurves. The mean value is 0.95 and the standard deviation 0.11 . The 108 objects are divided into bins of size 0.05 . Converting the stretch to $\Delta m_{15}(B)$ following Jha et al. (2006), a mean value of 1.13 and a standard deviation of 0.22 is obtained.

SNe Ia with poor lightcurves leaves 127 spectra of 108 different $\mathrm{SNe}$ Ia.

The epochs of the SN Ia spectra, calculated as the number of days in rest frame from $B$-band maximum light, are listed in Table A.2, and the sample distribution is shown in Fig. 3. In the table all epochs calculated from poor lightcurves are marked with a superscript " $p$ ". The uncertainty in the epoch is obtained as either the uncertainty in peak date obtained from SALT or through error simulations, whichever is bigger. The error simulations were performed by randomising the photometry within the error bars and making new SALT fits, after which the spread in the peak date was studied. The epoch errors include the uncertainty in the photometry, but they do not include any potential systematic uncertainties in the SALT fitting procedure.

The stretch distribution, shown in Fig. 4, is similar to the distribution of the Constitution set (Hicken et al. 2009), both regarding the mean value and the shape. The

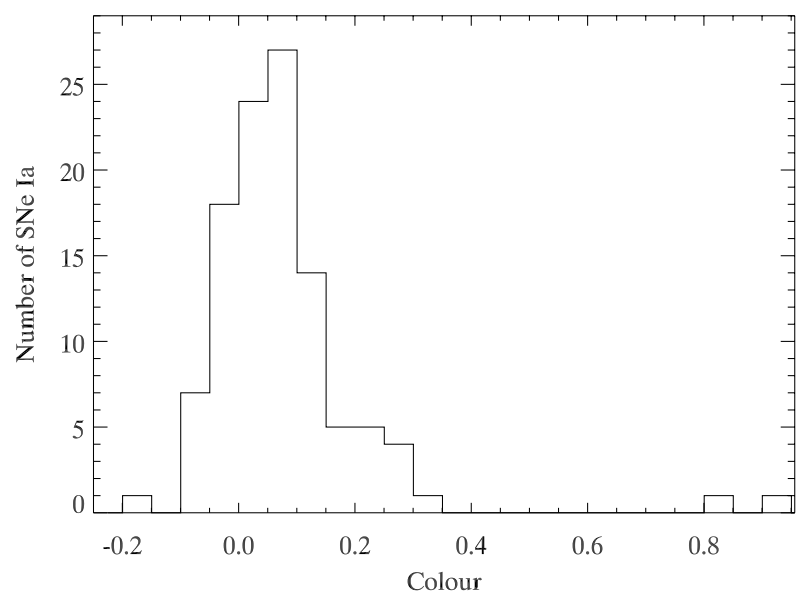

Fig. 5. Distribution of colour (SALT $c$ parameter) for the $\mathrm{SNe}$ Ia observed at the NTT and the NOT, excluding the SNe with poor lightcurves. The mean value is 0.08 and the median 0.07 . The standard deviation is 0.14 . The 108 objects are divided into bins of size 0.05 .

Kolmogorov-Smirnov (K-S) test gives a probability of $35 \%$, which is strong evidence against rejecting the assumption that the two samples of stretch belong to the same distribution. The distribution of colour (SALT $c$ parameter), presented in Fig. 5, has a K-S probability of $4 \%$ when compared to the distribution of the Constitution sample of Hicken et al. (2009). We conclude that the lightcurve properties of the SNe Ia in our sample are statistically compatible with the SN lightcurves previously used in cosmological analyses.

\subsection{Data archive}

The spectra are publicly available in electronic format ${ }^{3}$. In addition to the calibrated spectra, a corresponding error spectrum is also provided. Versions of the spectra are available both with and without host-galaxy subtraction. Additional information about the spectra on the website; observing conditions, object type, days since maximum brightness, redshift, etc. are available in Appendix A of this paper.

\section{Data reduction}

The spectroscopic data were reduced using the Image Reduction and Analysis Facility (IRAF) 4 and our own IDL routines. Below follows a step-by-step description of the reduction procedure.

Bias subtraction. Bias frames were taken on every night. No spatial variations were detected so we used the CCD overscan region to subtract the bias.

Flat fielding. The flat fields from the NTT observations had a peculiar feature at the blue end of the spectrum corresponding to wavelengths shorter than roughly $5200 \AA$ (see the left panel of Fig. 6). This is an effect from the zeroth order image of the grism which, while being outside the CCD area, still produced a visible glow on the detector. The behaviour was not present in any of

${ }_{3}$ http://www.physto.se/ linda/spectra/nttnot.html

${ }^{4}$ IRAF is distributed by the National Optical Astronomy Observatories, which are operated by the Association of Universities for Research in Astronomy, Inc., under cooperative agreement with the National Science Foundation. 


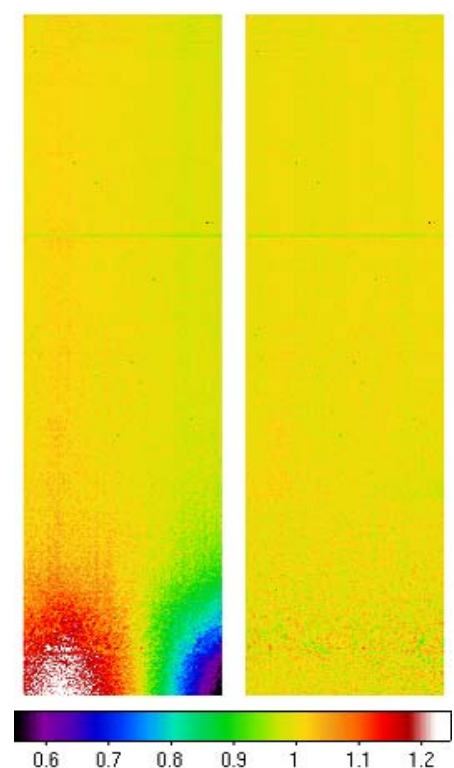

Fig. 6. A normalised flat field from the NTT before (left) and after (right) the correction for the zeroth order light was applied. The figure shows only one of the two chips of the CCD. The vertical direction is the dispersion axis and the horizontal direction is the spatial axis. The scales are the same for the two images.

the other frames and was removed from the flat fields by fitting a surface over the region. In the right panel of Fig. 6, the corrected and normalised flat is shown. Some horizontal lines can be seen in the flat field image around $7600 \AA$. These are absorption lines due to water vapour and molecular oxygen, and are caused by the long light path inside the instrument. This leads to a variation of the order of $3.5 \%$ in the normalised flat ${ }^{5}$.

For a given night of observation, one flat field was constructed for each grism/slit-width combination, which was then used to flat field the 2D spectral images of the standard stars and the $\mathrm{SN}$ candidates.

Spectral extraction. The SN spectra were extracted using the optimal extraction algorithm of Horne (1986). An extraction window was chosen in the spatial direction for a narrow wavelength range and was then traced for all wavelengths. In the cases when the SN was not well separated from the host galaxy, a small spatial extraction window was used to minimise contamination from host-galaxy light. For the sky background subtraction, two small spatial ranges were defined on each side of the $\mathrm{SN}$, and a fitted linear function was subtracted. For extended host galaxies, when the SN light was separated from the core of the host galaxy, this background fit also included host-galaxy light. Any residual host-galaxy light present in the spectra was removed at a later stage in the reduction process (see Sect. 6.2). When the combination of SN and host galaxy was complex, we experimented with different sizes of extraction apertures and higher orders of background fits, but without any significant improvement in the final result.

Wavelength calibration. For wavelength calibration, spectra of a helium-argon lamp were taken at the NTT and of a helium lamp at the NOT. For each object spectrum, an arc spectrum was

\footnotetext{
5 See the documentation about EMMI

wWw . eso.org/sci/facilities/lasilla/instruments/emmi
}

extracted with the same centring and trace as the object spectrum. A Chebyshev polynomial of fifth or sixth order was fitted to the identified wavelengths for the arc lamp emission lines. The solution was checked against locations of sky lines. The wavelength solution was then applied to the object spectrum.

Correction for second order contamination. When obtaining spectra over a wide wavelength range in one single exposure, contamination from second order diffraction could lead to an erroneous flux where there is an overlap of the orders. The second order contamination can be circumvented by using a blocking filter for blue light or by ignoring the red end of the spectrum. However, this reduces the wavelength range of the spectrum. Another method is to double the exposure time and divide the observations into a blue part and a red part. However, for faint objects which require long exposure times, this is not desirable. A method has been developed by Stanishev (2007) to correct spectra, obtained at the NOT using grism 4, for second order contamination during the reduction phase. With this method the full spectrum can be used. Observations were made at the NTT using grism 2 to derive the necessary information to correct spectra from that telescope too. To be able to correct for the second order contamination we need to (1) find out the wavelength relation between the first-order spectrum and the second-order spectrum and (2) find the ratio of the efficiencies of the grism for the two orders. The wavelength overlap of the two orders were determined using observations of arc lamps, together with blocking filters. To determine the flux relation between the two orders, bright blue stars were observed with and without orderblocking filters. Szokoly et al. (2004) have developed a similar method.

In extreme cases the second order contamination can be as strong as the flux at long wavelengths. The size and shape of absorption lines at these wavelengths can also be affected. This is illustrated in Fig. 7, where two examples of second order corrected spectra are shown, one for each telescope. If the second order contamination is not removed, systematic effects could be introduced since blue $\mathrm{SNe}$ are more affected by the contamination than red $\mathrm{SNe}$.

Flux calibration. To correct the object spectra for the wavelength dependent sensitivity of the detector, the spectra were flux calibrated using spectrophotometric standard-stars. For the NTT, the standard stars were observed with a slit width of 5 ". $^{\prime} 0$ and for the NOT with a slit of width 1". 3 . A wider slit width is used to make sure that the seeing does not affect the flux through the slit. All standard stars were observed at parallactic angle, to avoid effects from differential atmospheric diffraction. The standard-star spectra were processed in the same way as the object spectra.

Using IRAF, a sensitivity function was created for each instrument, slit width and observing session (of up to five nights). For the observations at the NOT during September and November 2007, a common sensitivity function was created since few standard star observations could be used. No significant variations with time were seen.

Corrections due to atmospheric extinction were implemented for La Silla and La Palma.

For a significant fraction of the SN observations, the parallactic angle was not used, causing differential slit losses due to atmospheric refraction. This problem will be discussed in detail in Sect. 5. 

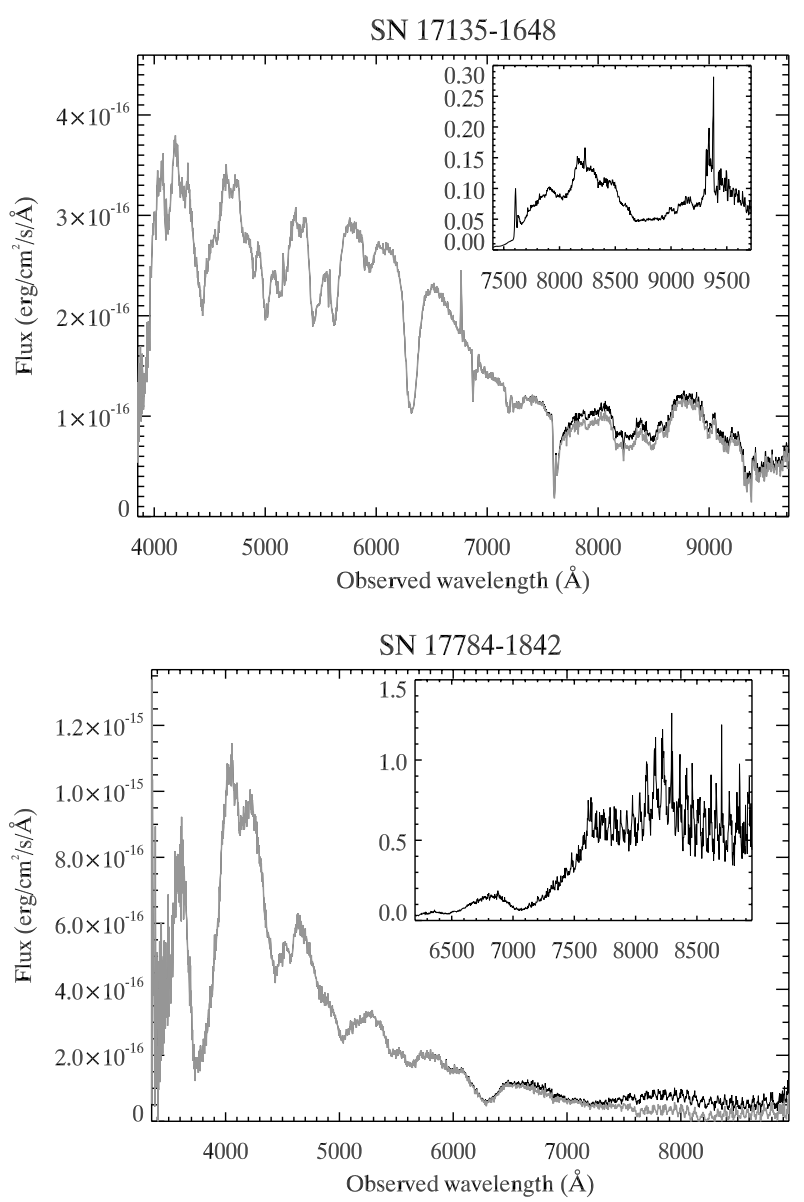

Fig. 7. Second order corrections of two SNe, ID 17135 (SN 2006qm) observed at the NTT (top panel) and ID 17784 (SN 2007jg) observed at the NOT (bottom panel). The observed flux is displayed in black and the corrected flux in light grey. The spectra have been flux calibrated. No corrections due to telluric absorption, Milky Way extinction or effects from host-galaxy light have been applied to the spectra shown here. The inset in each panel shows the relative correction with respect to the observed flux.

Telluric removal. The spectra have been corrected for telluric absorption. Using standard-star observations the telluric absorptions were isolated and then combined into one telluric spectrum for each slit width and night of observations. The SN spectra were then corrected through division of a scaled telluric spectrum. The scale factor was determined as the optimal weight for removing any telluric absorption near $7600 \AA$. This is the dominant telluric region, while not containing any interesting $\mathrm{SN}$ features for objects in our redshift range. In some cases no weight could be found such that the telluric spectrum obtained during the same night could remove the telluric feature. If another telluric spectrum obtained in close connection to the SN spectrum could do the matching, this was used instead. Some spectra could not be corrected perfectly using any telluric spectrum. The increased uncertainty in the spectra due to possible un-corrected telluric absorption was estimated by calculating the deviation from a spline fit through the $7600 \AA$ region, after correction. This error was then added to the SN error-spectrum.

Correction for dust extinction in the Milky Way. The spectra were corrected for dust extinction in the Milky Way using $R_{V}=3.1$ and the colour excess $E(B-V)$ from
Schlegel et al. (1998). The extinction was assumed to follow the Cardelli et al. (1989) extinction law modified according to O'Donnell (1994). According to Schlegel et al. (1998), the uncertainty on $E(B-V)$ is of the order of $16 \%$. The errors due to the uncertainty in $R_{V}$ and the uncertainty in the extinction law was found to be of less importance. The wavelength-dependent errors from the Milky Way correction were added in quadrature to the existing error spectrum.

\section{Differential atmospheric refraction and slit losses}

Differential atmospheric refraction (DAR) occurs when an observed image is spread out with wavelength along the direction toward the horizon due to the wavelength dependence of the refraction index of air (see e.g. Filippenko 1982; Cuby et al. 1998). As a result, objects centred on the slit for one wavelength, may be partially or even fully outside the slit for shorter and longer wavelengths. The effect can be minimised by (1) observing at a low airmass and (2) rotating the slit to the parallactic angle, where the slit is parallel to the direction of the atmospheric refraction, i.e. normal to the horizon. Other factors affecting the differential slit loss are: slit width, seeing, morphology of the observed object, spectral coverage and the wavelength(s) for which the object is centred.

The spectroscopic observations of SNe presented in this paper were typically not made at parallactic angle ${ }^{6}$. Instead, the slit orientation was most often chosen to simultaneously give a spectrum of the host galaxy. The differential atmospheric refraction (in arcseconds) for a certain wavelength with respect to a reference wavelength was estimated using the formulae by Owens (1967). The reference wavelength is the wavelength with which the SN had been centred on the slit. To calculate the fraction of the flux within the slit for each wavelength we modelled the SN flux with a Gaussian distribution using the seeing at the time of observations. In Fig. 8, we show one of the observed spectra with an insignificant host-galaxy contamination and a severe differential slit loss. The spectrum is shown together with a SN Ia template from Hsiao et al. (2007) (which we from now on will refer to as Hsiao templates) in its original form as well as affected by the estimated differential slit loss. The slit loss affected template is a much better fit to the observed spectrum. The estimated slit loss at very short wavelengths is exaggerated, which seems to be true for a large fraction of the theoretically calculated slit loss functions. In most cases such a direct comparison cannot be made due to the complex interplay of host-galaxy contamination and slit loss effects. In Table A.3 we indicate the estimated effect from the differential atmospheric refraction on the spectra at an observed wavelength of $4000 \AA$. It should be noted that this is only a rough estimate of the differential slit loss since many simplifications are used in the calculations and the centring over the slit was not perfect. For a non-negligible fraction of the NTT/NOT spectra, the differential slit loss is severe at short wavelengths. This is due to large airmasses and large differences between the observing angle and the parallactic angle. The median value of the airmass of the observations is 1.4 , with $39 \%$ of the observations having an airmass larger than 1.5. Large airmasses were unavoidable because of the position of the SDSS SN fields relative to the NTT and the NOT sites, during the observing season. Deviations from observations at the parallactic angle were also fairly large: nearly half of the spectra $(46 \%)$ were observed with $45^{\circ}$ or more from the parallactic

6 The observations of the standard stars were always made at parallactic angle. 

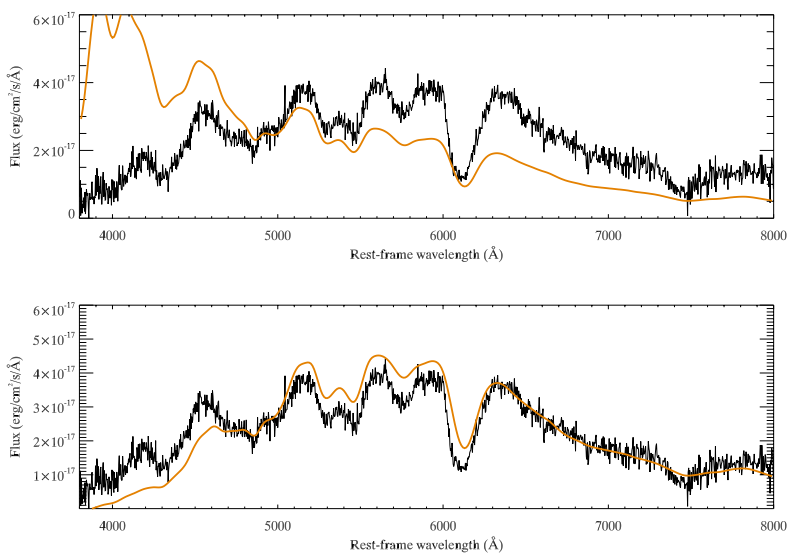

Fig. 8. The observed spectrum of ID 16287 (SPID 1449) 2 days past maximum brightness is shown in black together with the Hsiao template at the same epoch in orange (upper panel). In the lower panel the Hsiao template has been multiplied with the theoretically calculated differential loss due to the atmospheric refraction.

angle. The centring of the objects on the slit was done at wavelengths above $4600 \AA$, typically at $6500 \AA$.

A polynomial multiplier is included in the host-galaxy subtraction to account for the differential slit loss effects. However, these empirically determined corrections differ from the theoretically estimated functions described above since the polynomial also accounts for reddening.

\section{Host-galaxy contamination}

A large fraction of the obtained SN spectra are contaminated by galaxy light. We present below an estimation of the galaxy contamination in the spectra from photometry and a description of the host-galaxy subtraction method applied to the data.

\subsection{Estimating the degree of host-galaxy contamination}

The host-galaxy contamination in the observed spectra was estimated using multi-band SDSS photometry. To obtain an estimate of the galaxy light in the slit, the measured photometric surface brightness of the galaxy at the position of the SN was scaled to the area defined by the slit width times the width of the spectral extraction window. The expected SN flux in the observed spectra was calculated using the SDSS lightcurve, interpolated to the night the spectrum was taken. For objects where good lightcurve fits were available, these were used for the interpolation. The amount of SN flux through the slit was estimated by modelling the SN flux as a Gaussian distribution with the width determined from the seeing. The seeing variations between the different filters were estimated following Schroeder (1987) $\left(\propto \lambda^{-0.2}\right)$. In Table A.3, the estimated contamination in the $g$-band (observed frame) for each observed spectrum is listed. The contamination values are rough estimates and they are more likely over estimated since we assume that all galaxies are extended over the whole extraction window. Out of the spectra, $31 \%$ have an estimated contamination less than $20 \%$. The cases where extrapolation of the lightcurve was necessary are marked with a superscript of " $e$ " in the table. These values should be considered less reliable.

\subsection{Host-galaxy subtraction}

In the past, several different techniques have been used to subtract the host-galaxy contribution from observed spectra, such as $\chi^{2}$ based template fitting using the spectrum of the actual host galaxy or templates, either varying the contamination level or estimating it from photometry (e.g. Howell et al. 2005; Ellis et al. 2008). For the vast majority of the NTT/NOT spectra, no good quality spectra of the host galaxy at the location of the SN were accessible at the time of the work and thus a $\chi^{2}$ based fitting using a real galaxy spectrum was rejected. To model the galaxy, principal component analysis (PCA) was chosen over template spectra. A similar method has also been used by Zheng et al. (2008). There are also host-galaxy subtraction methods which separate the two components, SN och galaxy, in the two dimensional spectrogram (Baumont et al. 2008). However, this requires higher resolution of the data than that of the NTT/NOT data set.

For the NTT/NOT data set a number of different methods to subtract host-galaxy light from spectra were explored. We found the most stable method to be a principal component analysis based subtraction of galaxy eigencomponent spectra. For a large fraction of the moderately contaminated spectra (10-70\% contamination in the $g$-band) we found this method to yield good results.

It should be noted that some part of the host-galaxy light, at least for host galaxies with larger spatial extent on the CCD, were removed during a linear background fit in the reduction of the spectra. For these spectra the host-galaxy contamination that was fitted thus were the residuals from the reduction.

An additional difficulty in the host-galaxy subtraction was that a large fraction of the NTT/NOT spectra were not observed at parallactic angle (see Sect. 5), and were thus affected by a wavelength dependent flux loss.

In the PCA-based subtraction method described here, SN templates were used. A potential worry is that the usage of templates constructed from normal low-redshift SNe might affect the outcome of the subtraction. This concern and other concerns are addressed in Sect. 6.5.

PCA-based galaxy subtraction. The spectral energy distribution (SED) of the host galaxy was estimated through minimising the difference between the observed spectrum and a combination of a SN template and a set of galaxy eigencomponent spectra. The minimisation can be described with the formula

$f_{\text {fit }}(\lambda)=a_{0} s(\lambda) \cdot f_{\mathrm{SN}}(\lambda)+\sum_{i=1}^{n} a_{i} g_{i}(\lambda)$,

where $f_{\mathrm{SN}}$ is the $\mathrm{SN}$ template, $g_{i}$ the galaxy eigencomponent spectra, $s$ is a second degree polynomial and, $a_{i}$ weights which are fitted in the subtraction.

The galaxy eigencomponent spectra were created in a PCA analysis of 170000 SDSS galaxy spectra (Yip et al. 2004). The three most dominant eigenspectra have been shown to describe $99 \%$ of all galaxies ${ }^{7}$. The fit can be made arbitrarily complex through the inclusion of more galaxy eigencomponent spectra (to the existing three), but this extension was not used in the final studies since the fits did not improve significantly while the computational demands increase drastically. In the fit we included the constraint that the total galaxy flux must be positive.

7 Galaxy emission lines can have errors of up to $10 \%$ when using only three eigencomponent spectra (Yip et al. 2004). However, for our purposes it is enough with a well described continuum. 

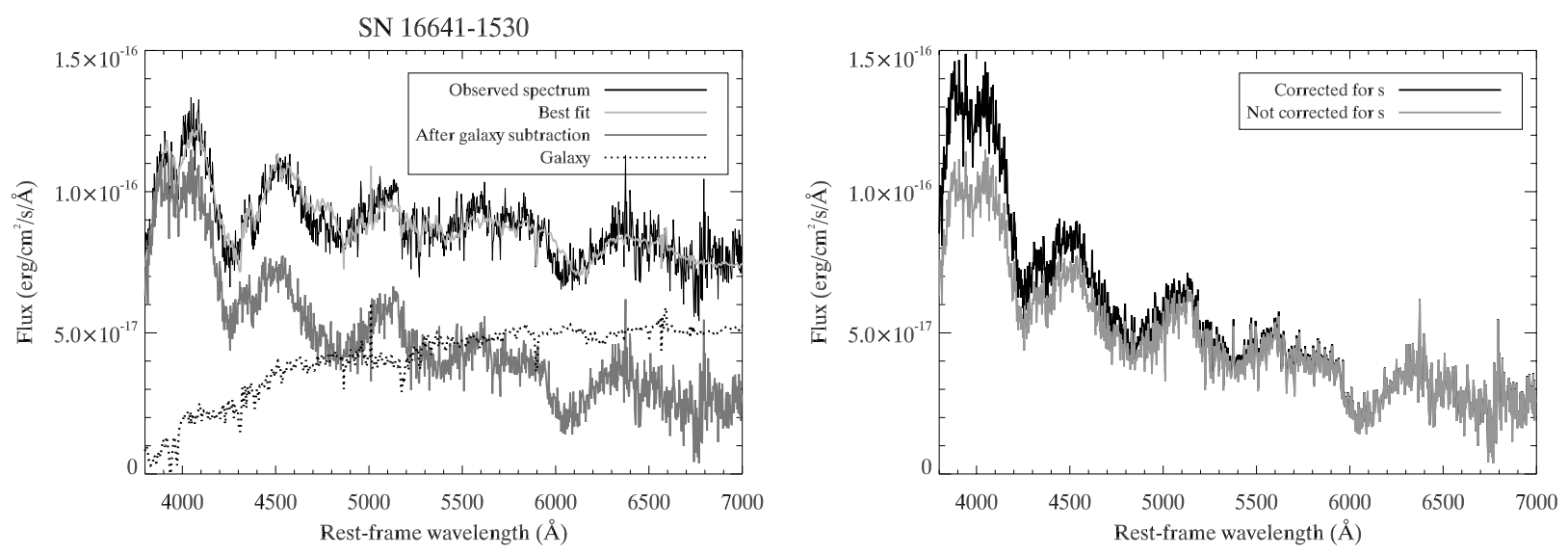

Fig. 9. The PCA-based host-galaxy subtraction for ID 16641 (SN 2006pr) at $z=0.132$. Left panel: the observed spectrum (black) is plotted together with the best fit (light grey). The galaxy template of the best fit is shown as a dotted line. The grey line shows the SN spectrum after subtraction of the estimated galaxy light. Right panel: the (host subtracted) SN spectrum is shown with and without correction for the wavelength dependent flux loss described by $s$ (black/grey). The host galaxy contamination estimated from photometry $(40 \%)$ agrees well with the fraction of host galaxy light indicated by the subtraction. The expected differential slit loss estimated following Sect. 5 (30\% at $4000 \AA$ ) also seems close to what the best fit in the host-galaxy subtraction indicates (compare the curves at $4000 \AA$ in the right panel).

For the SN template in the fit we tried all Hsiao templates with epochs \pm 5 days from the spectral epoch as obtained from the photometric lightcurve. We also included templates of more peculiar SNe, SN 1991bg and SN 1991T (Nugent et al. 2002), to study how well the Hsiao templates worked and to, possibly, find peculiar SNe in our sample. None of our spectra were well fitted with the SN 1991bg template (there will be a bias against such objects due to their faintness). Some spectra had better fits with a SN 1991T template. The difference in the features sizes between the subtraction with the Hsiao template and the SN 1991T template was small in these cases.

To the SN template a second degree polynomial was multiplied which was introduced to account for reddening (e.g. due to host galaxy dust extinction) and differential slit loss effects. During observations, the object was centred on the slit in the red part of the spectrum, and thus differential slit losses due to atmospheric refraction (see Sect. 5), when present, predominantly affects the blue end (as would extinction). The second degree polynomial was fixed to have $s \equiv 1$ at the wavelength $\lambda_{1}=6600 \AA$ and $s<1$ for all other wavelengths. The value of $\lambda_{1}$ was chosen to correspond to the wavelength where most spectra were centred on the slit. The function describing the differential slit loss is asymmetric around this wavelength, but since the fit during host-galaxy subtraction was only made between $4000 \AA$ and $6000 \AA$, the behaviour of the slit loss function at longer wavelengths did not affect the fit. The polynomial was only multiplied with the SN SED, and not with that of the galaxy. The reason for this was that since galaxies are not point sources, they are less affected by differential slit losses. A separate polynomial could be modelled for the fitted galaxy, but this would be both hard to evaluate and too computationally demanding, considering the small effect. A test was performed where the same polynomial was added both to the galaxy and the $\mathrm{SN}$, but this did not improve the quality of the fits.

The code for the PCA-based host-galaxy subtraction has only been applied to SNe Ia with known redshifts and a good lightcurve (photometry both before and after peak brightness). Two sample subtractions for one moderately and one highly host-galaxy contaminated spectrum can be seen in Figs. 9 and 10 .

\subsection{Host-galaxy simulations}

Set-up. To evaluate the subtraction methods, a large number of simulated "fake" spectra were constructed and run through the subtraction pipelines. Each fake spectrum was constructed through a combination of a SN and a galaxy spectrum, redshifted to some distance. Reddening and differential slit loss were added to the SN spectrum. Finally, noise was added. All parameters (redshift, spectral epoch, host-galaxy contamination, reddening, differential slit loss) were drawn from distributions that match the NTT/NOT data set. In constructing these fake spectra we avoided all templates/functions that were used in the host-galaxy subtraction pipeline (as this would make the fit trivial). More details about the different components of the fake spectra are given below:

- Supernova spectra. All SN spectra that were used to construct fake spectra have high S/N and low host-galaxy contamination. Their epochs are similar to the ones of the NTT/NOT spectra. Seven different spectra of normal SNe Ia were used: six of SN 2003du (epochs $-6,-2,4,9,10,17$ ) (Stanishev et al. 2007) and one of SN 1998aq at peak brightness (Branch et al. 2003). Furthermore, two spectra of the sub-luminous SN 1999by (epochs -5 and 3) (Garnavich et al. 2004) were used and two of the peculiar and luminous SN 1999aa (epochs -5 and 0) (Garavini et al. 2004).

- Reddening. Reddening was added to the SN spectrum using the Cardelli et al. (1989) extinction law with a totalto-selective extinction ratio $R_{V}$ of 2.1 and a colour excess $E(B-V)$ drawn from the distribution of $E(B-V)$ obtained from the NTT/NOT lightcurve fits. The lower value of $R_{V}$ compared to the Milky Way average was chosen to agree with the values often derived when looking at SNe Ia (see e.g. Altavilla et al. 2004; Nobili \& Goobar 2008).

- Galaxy spectra. In a first simulation series, four galaxy templates of varying type (elliptical, S0, Sa and Sb) from Kinney et al. (1996) were used together with three real galaxy spectra (host galaxy spectra for ID 7527, 13840 and 15381) observed at the NTT at the same time as the SN spectra presented here. The contamination level was randomly chosen between 0 and $70 \%$ for the $g$ band. These simulations were later extended in a second series, where 50 randomly chosen 

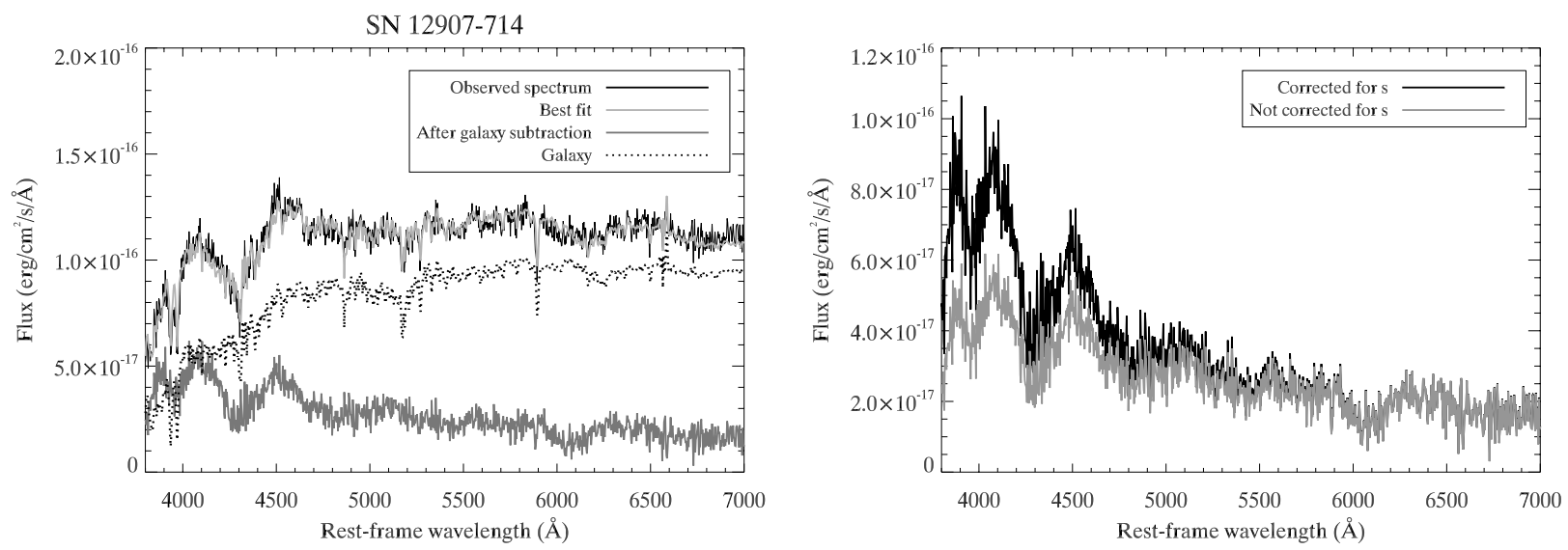

Fig. 10. The PCA-based host-galaxy subtraction for the highly contaminated ID 12907 (SN 2006fv) at $z=0.132$. Left panel: the observed spectrum (black) is plotted together with the best fit (light grey). The galaxy template of the best fit is shown as a dotted line. The grey line shows the SN spectrum after subtraction of the estimated galaxy light. Right panel: the (host subtracted) SN spectrum is shown with and without correction for the wavelength dependent flux loss described by $s$ (black/grey). The host galaxy contamination estimated from photometry (80\%) agrees well with the fraction of host galaxy light indicated by the subtraction. The expected differential slit loss, see Sect. 5 (30\% at $4000 \AA$ ) also seems compatible to what the best fit in the host-galaxy subtraction indicates (compare the curves at $4000 \AA$ in the right panel).
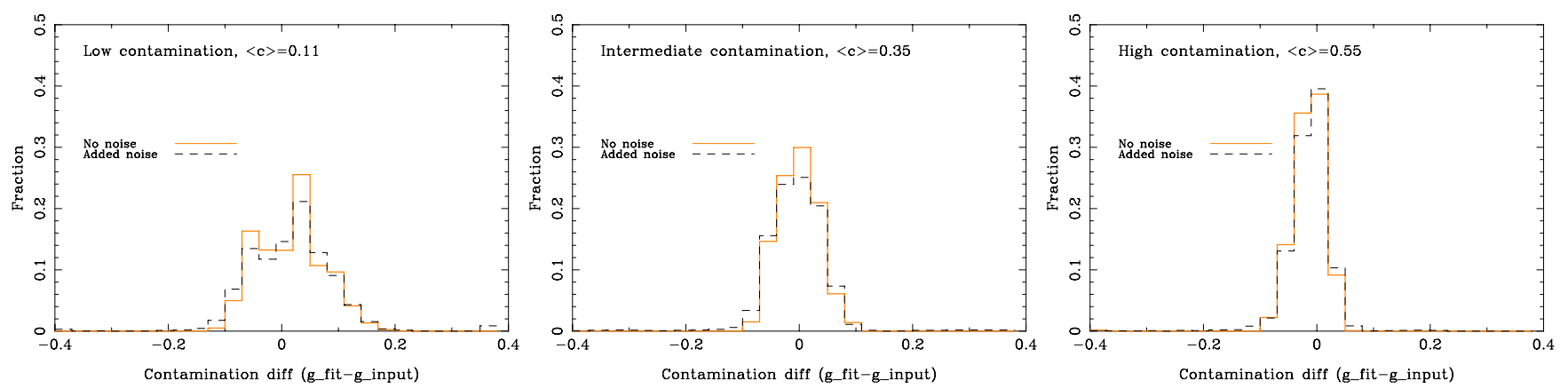

Fig. 11. The difference between the $g$-band host-galaxy contamination that was introduced in the construction of fake spectra ( $g$ _input) and the contamination that was obtained during host-galaxy subtraction ( $g$ _fit) for three different bins in $g \_$input. The mean contamination for each bin is given in the respective panel as $\langle c\rangle$. The contamination was calculated as the percentage of the total flux in the observed SDSS $g$ band which comes from the galaxy. The solid histogram corresponds to simulations without added noise while the dashed line corresponds to where noise is added. The dispersion is largest for small input contaminations. The number of outliers increase from $0.9 \%$ to $2 \%$ when noise is added.

SDSS galaxy spectra were used. Figures displayed here are based on the first run, but results are very similar when including the second set of galaxy spectra.

- Redshifts. The object redshift was randomly drawn from the NTT/NOT redshift distribution.

- Differential slit loss. To the SN spectra we multiplied functions modelling the expected differential slit loss due to atmospheric refraction for typical NTT/NOT observing conditions (see Sect. 5). These effects range from insignificant to severe. The slit loss functions are applied to the SN spectra but not to the galaxy spectra.

- Noise. An S/N value was randomly chosen from the NTT/NOT spectral S/N distribution. Poisson noise was then added to the spectrum until the target $\mathrm{S} / \mathrm{N}$ was achieved. The shape of the Poisson noise was determined as a linear combination of the input spectrum and a randomly chosen NTT/NOT sky spectrum. The linear combination was determined such that the highest $\mathrm{S} / \mathrm{N}$ value in the NTT/NOT sample corresponded to no contribution from sky noise, the lowest $\mathrm{S} / \mathrm{N}$ corresponded to complete dominance by sky noise and intermediate values to a combination of the two error sources. Simulations were done both with and without noise. As can be expected, added noise increased the dispersion around the true solution, but no significant bias effects were detected.

For the simulations without noise, 5000 fake spectra were constructed and about twice as many for the simulations with noise.

Evaluation of the host-galaxy simulations. The simulations can be evaluated using a range of different tests. For all fake spectra we compared the input SN and galaxy spectra with the SN and galaxy as estimated by the host-galaxy subtraction pipeline. In Nordin et al. (2011) we study how spectral indicators are affected by the host-galaxy subtraction as a function of contamination level. A more direct way of evaluating subtractions is to compare the input contamination with what is obtained from the subtraction output. In Fig. 11 we show how the difference between input and fitted output contamination varies with input contamination (in the $g$ band). The dispersion is largest for low contamination levels. When studying the contribution from different SN templates, it is found that for contamination levels roughly below $20 \%$, some SN templates yield a small offset. This offset has its origin in a combination of two effects: first, some of the SN templates most likely contain some host galaxy light. This would be seen as a slight bias for low contamination 
levels, but should thus not be interpreted as a bias of the host subtraction method. Second, a bias can occur if the SN spectrum is imperfectly matched to the Hsiao template. The offset decreases with contamination level. For objects with very low contamination it can thus be advantageous not to subtract the estimated host galaxy light since the correction likely is smaller than the uncertainty in the correction. No trend with galaxy template or slit loss function could be seen. When noise is added to the fake spectra, the number of failed subtractions increase, from $0.9 \%$ to $2 \%$. These failures are usually evident through a visual inspection of the subtraction. The percentage agrees with what is seen when evaluating subtractions of real SNe. These events likely correspond to random noise mimicking host galaxy or SN features.

\subsection{Alternative methods}

Several other host-galaxy subtraction methods were tried: varying the galaxy eigenspectra (their number and source), varying the limitations of the eigenspectra, using a large set of SN templates and using SDSS photometry to fix the fraction of galaxy light as well as the proportions of different galaxy eigenspectra. These alternative methods were found to be either less stable than the method above or equivalent (but involving more computational effort). It should be noted that for some individual $\mathrm{SNe} \mathrm{Ia}$, an alternative method might perform better. Alternative host-galaxy subtractions of spectra can be provided upon request.

\subsection{Discussion on the host-galaxy subtraction}

The host-subtraction method is not successful for all NTT/NOT $\mathrm{SNe}$, especially for very faint or contaminated cases. It is, however, not obvious how to judge when a subtraction does fail. Both visual inspection and $\chi^{2}$ tests only indicate how well the subtracted spectrum match the local template, and can thus not be trusted to completely specify which subtractions succeed.

Some of the main worries with the subtraction technique used here are: (i) the observed SNe could differ from the local $\mathrm{SN}$ templates used e.g. if it is a peculiar type; (ii) the true galaxy contribution to the spectrum could be badly described by the use of galaxy eigencomponent spectra since the complete galaxy is not recorded in the slit; and (iii) the approximation of slit loss and reddening with a polynomial (applied to the SN but not to the galaxy SED) is a simplification. Regarding the first point, object typing was also performed prior to galaxy subtraction, which means that any truly odd SN would have been spotted at this stage unless it is heavily contaminated by galaxy light. Furthermore, the SN template is only used in the choice of what galaxy spectrum to remove and the number of bins used in the fit is large compared to the number of parameters which are fitted and thus the risk of affecting the spectrum severely is small. Regarding the second point, the three most dominant eigenspectra have been shown to be able to describe $99 \%$ of all galaxies (Yip et al. 2004), which means that they should be sufficient to describe our host galaxies as well. The third point, as well as the host-galaxy subtraction code in general, was tested through an extensive number of simulations as described in Sect. 6.3. The host-galaxy subtraction does fail in roughly one percent of all runs, but these cases usually fail completely, yielding unphysical results, and are thus easily detected.

In Nordin et al. (2011) we further test the host-galaxy subtraction code, by examining the effects on spectral features, in particular on pseudo-equivalent widths. We also compare the trends of pseudo-equivalent widths for the full sample with the subsample of spectra with low host-galaxy contamination and conclude that no bias is introduced.

\section{Typing}

The object spectra have been typed using SNID (SuperNova IDentification; Blondin \& Tonry 2007), version 5.0. This algorithm cross-correlates the unknown spectrum against a set of template spectra. The template database includes SN spectra of different types and ages as well as non-SN spectra of galaxies, AGNs and stars. The redshift of the object can either be varied or fixed. In this version of the code, SNID distinguishes between the following types and subtypes (within parenthesis): Ia (Ia-norm, Ia-91T, Ia-91bg, Ia-csm, Ia-pec), Ib (Ib-norm, Ib-pec, IIb), Ic (Ic-norm, Ic-pec, Ic-broad), II (IIP, II-pec, IIn, IIL), Not-SN (AGN, Gal, LBV, M-star). The Ia-pec group consists of SNe of the type SN 2000cx and SN 2002cx. As a figure of merit for the classification we have used the rlap parameter (see Blondin \& Tonry 2007, for a definition). A good correlation was considered to be obtained if rlap was greater or equal to five. Following Foley et al. (2009) we made four runs with SNID to determine the type, subtype, redshift and age, one at a time. This was done in the following manner:

- First we attempted to determine the type. A type was identified if more than $50 \%$ of the templates with a good correlation belonged to this type and the best-match SN-template (highest rlap value) had the same type. When the redshift of the SN was known, we restricted the redshift range of SNID to this redshift $( \pm 0.02)$.

- If it was possible to determine the type, we continued with trying to determine the subtype. We fixed the type and the redshift (if it was known). A subtype was considered to be identified if more than $50 \%$ of the templates with a good correlation belonged to a specific subtype and the best-match spectrum was of the same subtype.

- Regardless if a subtype was identified or not, we determined the best-fit redshift. We now locked SNID to the templates of the identified type or subtype and fitted for the redshift. The SNID redshift was defined as the median of the redshifts belonging to templates with a good correlation. The error was given as the standard deviation.

- To determine the age, the type was fixed to the identified type or subtype. If we had a spectroscopic redshift, we fixed the redshift in SNID to this value. Otherwise we used the value obtained in the step before with a redshift error of 0.02 . The SNID age was then defined as the median of all ages belonging to templates with a good correlation. The error of the age was given as the standard deviation of these values.

If the determined type and redshift were inconsistent in that the preferred type was an M-star or an LBV, while the redshift was too high for such an object to be detected, the type was set to unknown.

A problem with this method is that the composition of the template database affects the possibilities to detect different types. The SNID database has few spectra of core-collapse SNe and peculiar SNe Ia which makes these objects harder to type. It is also less effective for low $\mathrm{S} / \mathrm{N}$ spectra than for those with high $\mathrm{S} / \mathrm{N}$.

The outcome of the SNID analysis is presented in Table A.2. The typing for each spectrum is presented in the column 
NTT/NOT type. When the type was determined using the hostgalaxy subtracted spectrum, this is marked in the column for notes with the addition of an $s$. In some cases a spectrum only had a good match using SNID with one particular object in the database. In these cases we have marked this in the table. When no type could be determined this is marked with "-".

The consistency in typing when several spectra of the same object existed was good. If a type could be determined, the same type was always obtained. In some cases, one of the spectra was left without a type due to, for example, noise making the classification difficult or it was classified as a galaxy, due to the faintness of the SN at that epoch.

After a visual inspection of the spectra, the typing from SNID was changed for 36 spectra. The majority of these, 20, could not be typed by SNID but could be clearly typed by visual inspection. There was also a group of spectra, 11, which had been typed by SNID, but with only few well matched template spectra, and, in the visual study, they were found to be of such low quality that it was not possible to say with certainty what the type was. Three spectra had a type which was changed, one from $\mathrm{a} \mathrm{Ib}$ to a Ib/c and two from a Ia to a possible Ia. There were also two cases where the type was kept, but the subtype was removed. The spectra whose type was changed following the visual check are marked with a $v$ in the column for notes in the table.

The NTT/NOT objects also have an SDSS typing based on the NTT and the NOT spectra in combination with spectra taken at other telescopes as a part of the SDSS-II SN Survey. This type is presented in Table A.2 under the column SDSS type. Due to the faintness of the SN in some spectra, not all NTT and NOT spectra could be typed and some were typed as galaxies, while the overall SDSS typing is a SN, based on spectra at other epochs from other telescopes.

In Fig. 12 the redshifts obtained from SNID are compared with the SDSS object redshifts from Zheng et al. (in prep.), which mainly come from the SDSS DR7 catalogue and measurements of galaxy lines in spectra obtained as a part of SDSS-II. Some are determined from SN features (see Sect. 3). There is a good agreement between the SNID redshifts and the SDSS redshifts, with a dispersion of 0.005 and a negligible bias. No dependence with redshift is detected.

In Fig. 13, the ages of the spectra as estimated with SNID are compared with the ages obtained from the lightcurves, $\left(\mathrm{MJD}_{\mathrm{spec}}-\mathrm{MJD}_{\max }\right) /(1+z)$, for the spectra which we have classified as $\mathrm{SNe}$ Ia. A dispersion of 4 days was obtained. It should be noted that for young SNe, SNID generally estimates an older age compared to the SALT lightcurve fit and that the errors in the age from SNID are typically overestimated, especially at later epochs. This was also pointed out by Blondin \& Tonry (2007). Since the estimated age error bars are symmetric (estimated as the standard deviation) and in many cases overestimed, there will be some cases where the minimum allowed age (from the error bar) will be unphyically low. We have not used any prior on the age from the lightcurve, which cause the large error bars and the bias in the epoch estimates at early epochs. It should be noted that the error bars, in a few cases, also could be underestimated. This occurs when the observed spectrum is only well fitted with few template SNe and these template epochs do not sample all different ages of a SN. One example is ID 16838 which is only well fit by spectra in the library of SN 1998S. For one spectrum (ID 20142), no error was estimated since only one template spectrum could fit the observed spectrum. We also test the hypothesis that the evolution of spectral features scale with the stretch factor, by multiplying the age obtained from SNID with $s$. The bias is slightly improved, while the spread increases.

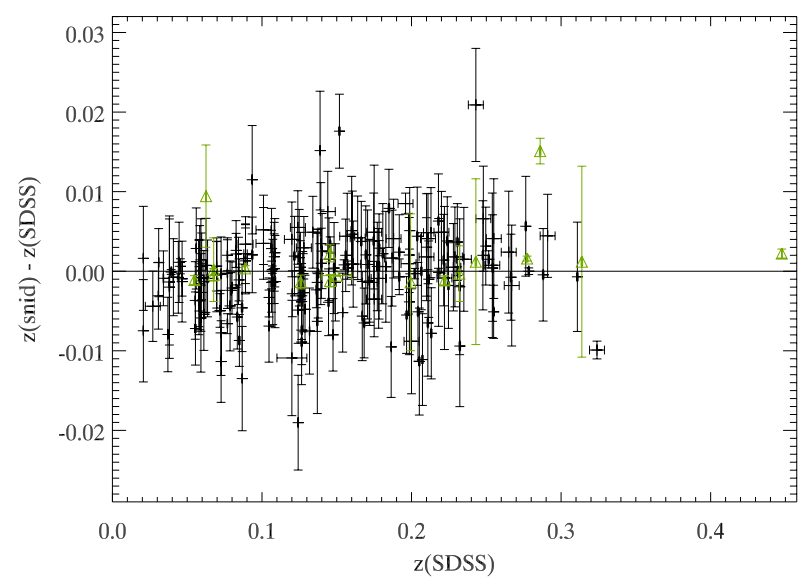

Fig. 12. A comparison of the SDSS object redshifts with the redshifts determined using SNID. The vertical error bars show the uncertainty in the SNID redshifts. The horizontal error bars show the uncertainty in the SDSS object redshifts. In most cases these are too small to be visible. All redshifts determined from $\mathrm{SN}$ features have an error of at least 0.005 . The rms of the distribution is 0.005 (with a negligible bias). SNID redshifts which are calculated using less than five well matched spectra are marked with green triangles.

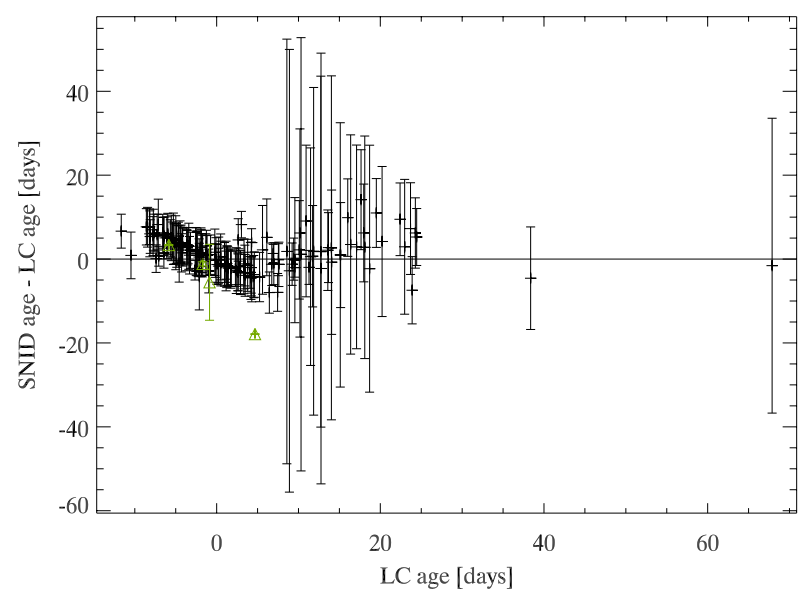

Fig. 13. A comparison of the age obtained using SNID and the age obtained from the lightcurve fit using SALT. The age is here defined as the number of days in rest frame since $B$-band maximum. SNID ages which are calculated using less than five well matched spectra are marked with green triangles.

\section{Some special objects}

Possible non-normal SNe la. Using SNID we find that the spectrum of ID 16333 (SN 2006on) is only well described by spectra of SN 1991T. The fit with SN $1991 \mathrm{~T}$ at 5 days before maximum brightness is shown in Fig. 14. The peak brightness of the $\mathrm{SN}$ is well constrained by the lightcurve fit (the stretch and colour less so) and we find a peak $B$ absolute magnitude of -19.06 . The obtained stretch was 0.94 and the SALT $c$ colour 0.19 . Correcting the peak magnitude for stretch and colour, following $M_{B}+\alpha(s-1)-\beta c$, gives a peak magnitude of -19.6 , which is consistent with a SN 1991T-like SN.

The spectrum of ID 17176 (SN 2007ie) has strong indications of being a SN 2002cx-like object. Using SNID, the spectrum is best fit with a spectrum of SN 2002cx. However, also other classes of SNe Ia give sufficiently good fits to the spectrum, when allowing for a variation of 0.02 in redshift. In Fig. 15, some of the SNID fits with different template spectra are shown. 


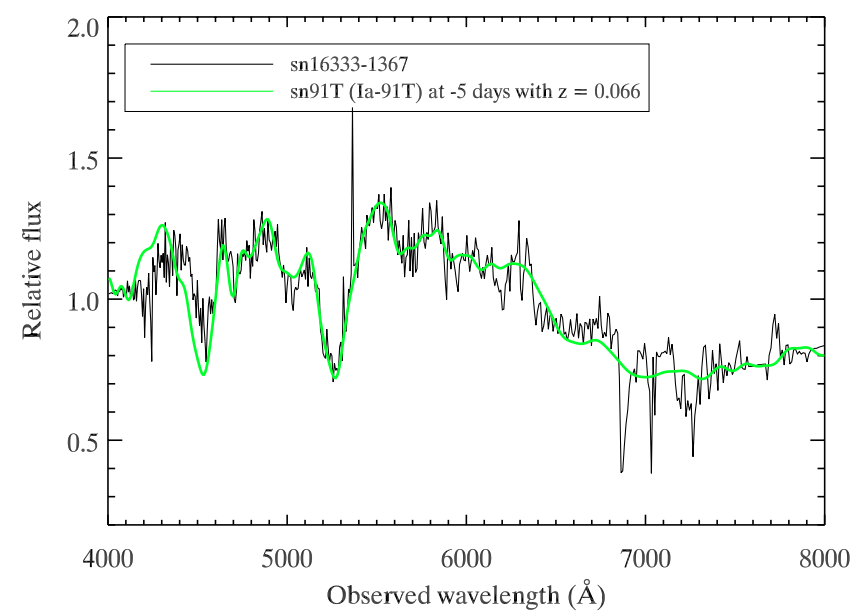

Fig. 14. SNID fit for ID 16333 (SN 2006on) with a SN 1991T template at 5 days before lightcurve maximum.

The spectrum of SN 2002cx is a better fit to the double peaks at an observed wavelength of $5100 \AA$ and $6100 \AA$ than the other spectra (normal SN Ia, SN 1991 T and SN 1991bg). Furthermore, the fit with the spectrum of SN 2002cx gives a better match with the redshift of the SN as obtained from SDSS DR7. If we reduce the allowed redshift range in SNID to \pm 0.01 , SNID identifies the spectrum as a SN 2002cx-like object. By comparing the spectrum with the ones by Sahu et al. (2008) of SN 2005hk, which also is a SN 2002cx-like SN, we identify absorption by $\mathrm{Fe}$ II $\lambda$ 25018, $\mathrm{Fe}$ II $\lambda$ 25535, Fe II $\lambda 6149$ and $\mathrm{Fe}$ II $\lambda 6247$. The velocities in our spectrum for these lines are lower by $7-35 \%$ compared to a spectrum of SN 2005hk at 24 days past peak brightness. Compared to the spectrum at 38 days past peak, the velocities varies from being lower by $26 \%$ to being higher by $9 \%$. The lightcurve, in this case, is not well constrained due to lack of photometry before maximum brightness. The best-fit absolute peak magnitude in the $B$-band from SALT is -18.2 , which is comparable to what is expected for SN 2002cx-like objects.

ID 19149 (SN 2007ni) is best fit with a SN 1991T-like spectrum according to SNID. It also has great similarities with SN 2002cx-like and SN 2000cx-like spectra, but not with spectra of normal SNe Ia. Comparisons from SNID with different templates are shown in Fig. 16. The lightcurve fit is well constrained and the obtained absolute peak magnitude in $B$ is -19.47 , which is somewhat brighter than a normal SN Ia. We could thus exclude an SN 2002cx interpretation. The stretch and SALT $c$ colour are 1.04 and 0.09 , respectively.

ID 20978 (SN 2007rl) is best fit in SNID with a spectrum of SN 2000cx. However, due to its high redshift, it is well fitted by many different templates. The peak brightness is well constrained by the photometry, while the stretch and colour are less so due to few observations past peak brightness. The absolute peak $B$ band magnitude is -19.6 .

Possible SNe IIn. In the spectral fitting we find that the spectrum of ID 16838 is best fit with a SN IIn, similar to SN 1998S. Another potential SN IIn is ID 16668 (SN 2006pu).

Stellar tidal disruption event. The object with SDSS identification 17237 has been suggested to be a stellar tidal disruption event (van Velzen et al. 2010), an event where a star passes close to a supermassive black hole in the centre of the galaxy without being completely destroyed.

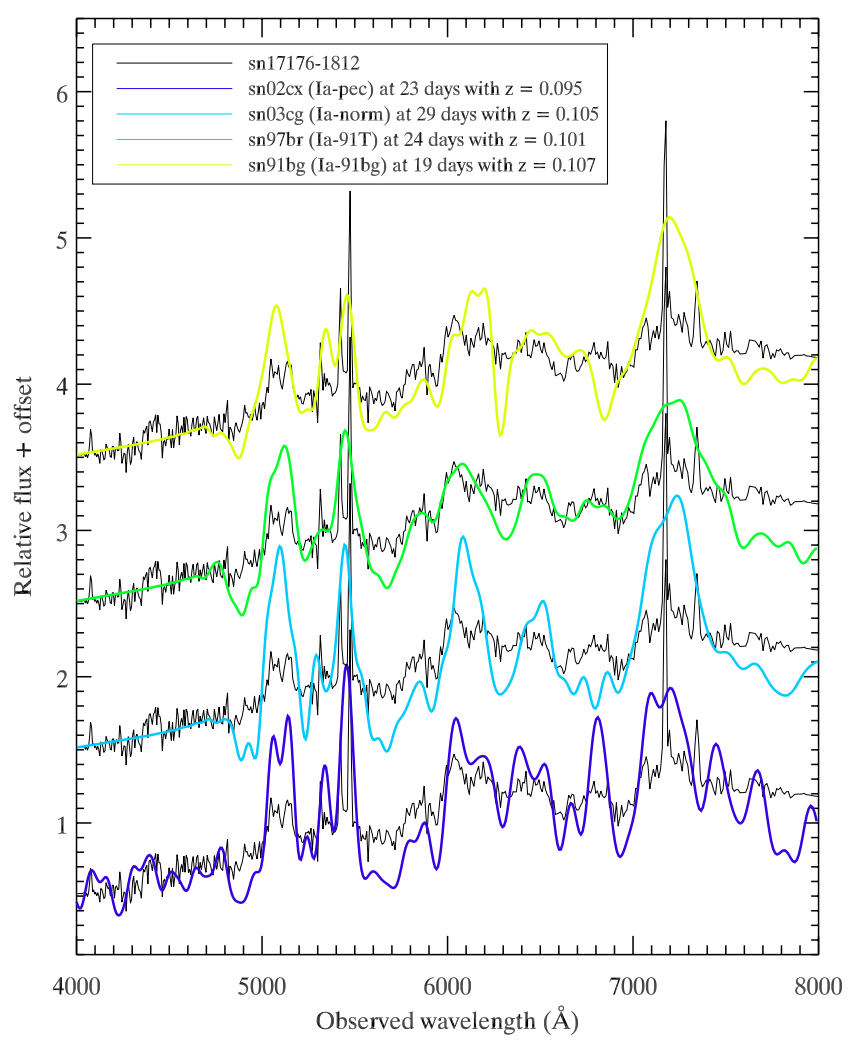

Fig. 15. The observed spectrum of ID 17176 (SN 2007ie) in black together with different template spectra as indicated in the legend. In the fit, the redshift has been fixed to 0.0935, the redshift of the object as obtained from SDSS DR7, with an allowed variation of 0.02. The fit with the spectrum of SN 2002cx gives the best match and the closest redshift compared to the one of the host galaxy.

\section{Summary}

We have presented observations and reductions of 290 spectra observed at the NTT and the NOT as a part of a programme to classify SNe discovered by the SDSS-II. The selection procedure, the observing strategy and the reductions have been performed in a coherent and well documented manner. The spectra have been corrected for telluric absorption. Objects classified as $\mathrm{SNe}$ Ia were processed through a host-galaxy subtraction pipeline. The host galaxy SEDs were estimated using a PCAanalysis where the difference between the observed spectrum and a combination of a SN template and galaxy eigencomponent spectra were minimised. A polynomial was multiplied to the SN templates to account for reddening effects as well as differential slit loss due to atmospheric refraction. The host-galaxy subtraction pipeline was evaluated using extensive simulations mimicking the distribution of parameters for the NTT/NOT data set. Furthermore, careful estimates of uncertainties have been made, thus making our data well-suited for systematic studies.

The spectra were classified using SNID, a template-matching algorithm. The age of the spectra estimated using SNID was compared to the age obtained from the lightcurve, in the case of SNe Ia, and was found to agree well, with an age dependent bias of a few days and an overall dispersion of 4 days. The typing was revised using visual inspection and changed in some cases; typically when the SNID classification was unreliable due to few matched spectra or when the spectrum could be typed by visual inspection but not with SNID. 


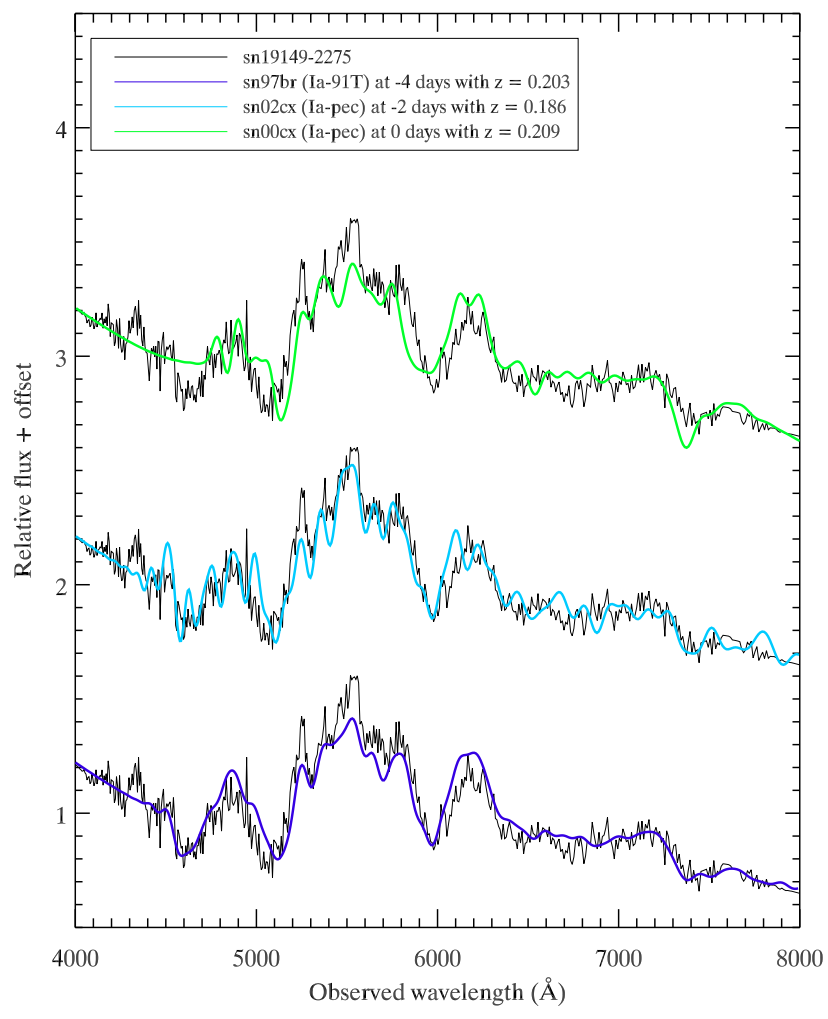

Fig. 16. The SNID fit of ID 19149 (SN 2007ni) with the observed spectrum in black and different template spectra in colour as indicated in the legend.

In total, 207 spectra of 172 different confirmed SNe were obtained at the NTT and the NOT for the SDSS-II SN program. Out of these, 169 are SN Ia spectra, and some of these (the ones which will survive the strict cosmology lightcurve cuts) will be part of a larger sample to be included in the three year SDSS-II SN Hubble diagram. Four potential peculiar SNe Ia were identified from their spectra. ID 16333 (SN 2006on) and 19149 (SN 2007ni) are potential SN 1991T-like objects, while ID 17176 (SN 2007ie) is a potential SN 2002cx object. ID 20978 (SN 2007rl) is identified as a peculiar SN Ia using SNID, but due to its high redshift, it is difficult to determine a subtype. The sample also includes 26 spectra of $23 \mathrm{SNe}$ II and 12 spectra of $8 \mathrm{SNe} \mathrm{Ib/c.}$

The reduced spectra are made public together with the estimated uncertainty for each wavelength. For the subsample of SNe Ia, host-galaxy subtracted spectra are provided. All this data is accessible from the webpage: http: //www . physto. se/ linda/spectra/nttnot.html.

Acknowledgements. The authors would like to thank Johan Fynbo, Christa Gall and Christina Henriksson who all helped out at the NOT observations. L.Ö is partially supported by the Spanish Ministry of Science and Innovation (MICINN) through the Consolider Ingenio-2010 program, under project CSD2007-00060 "Physics of the Accelerating Universe (PAU)". V.S is financially supported by FCT Portugal under program Ciência 2008. The Oskar Klein Centre is funded by the Swedish Research Council. The Dark Cosmology Centre is funded by the Danish National Research Foundation. Funding for the SDSS and SDSS-II has been provided by the Alfred P. Sloan Foundation, the Participating Institutions, the National Science Foundation, the US Department of Energy, the National Aeronautics and Space Administration, the Japanese Monbukagakusho, the Max Planck Society, and the Higher Education Funding Council for England. The SDSS Web Site is http://www.sdss.org/. The SDSS is managed by the Astrophysical Research Consortium for the Participating Institutions. The Participating Institutions are the American Museum of Natural History, Astrophysical Institute Potsdam, University of Basel, University of Cambridge, Case Western Reserve University, University of Chicago, Drexel University,
Fermilab, the Institute for Advanced Study, the Japan Participation Group, Johns Hopkins University, the Joint Institute for Nuclear Astrophysics, the Kavli Institute for Particle Astrophysics and Cosmology, the Korean Scientist Group, the Chinese Academy of Sciences (LAMOST), Los Alamos National Laboratory, the Max-Planck-Institute for Astronomy (MPIA), the Max-PlanckInstitute for Astrophysics (MPA), New Mexico State University, Ohio State University, University of Pittsburgh, University of Portsmouth, Princeton University, the United States Naval Observatory, and the University of Washington. The paper is partly based on observations made with the Nordic Optical Telescope, operated on the island of La Palma jointly by Denmark, Finland, Iceland, Norway, and Sweden, in the Spanish Observatorio del Roque de los Muchachos of the Instituto de Astrofisica de Canarias. The data have been taken using ALFOSC, which is owned by the Instituto de Astrofisica de Andalucia (IAA) and operated at the Nordic Optical Telescope under agreement between IAA and the NBI. The paper is partly based on observations collected at the New Technology Telescope, operated by the European Organisation for Astronomical Research in the Southern Hemisphere, Chile.

\section{References}

Altavilla, G., Fiorentino, G., Marconi, M., et al. 2004, MNRAS, 349, 1344 Balland, C., Baumont, S., Basa, S., et al. 2009, A\&A, 507, 85

Baumont, S., Balland, C., Astier, P., et al. 2008, A\&A, 491, 567

Blondin, S., \& Tonry, J. L. 2007, ApJ, 666, 1024

Blondin, S., Dessart, L., Leibundgut, B., et al. 2006, AJ, 131, 1648

Branch, D., Garnavich, P., Matheson, T., et al. 2003, AJ, 126, 1489

Bronder, T. J., Hook, I. M., Astier, P., et al. 2008, A\&A, 477, 717

Cardelli, J. A., Clayton, G. C., \& Mathis, J. S. 1989, ApJ, 345, 245

Cuby, J. G., Bottini, D., \& Picat, J. P. 1998, in Society of Photo-Optical Instrumentation Engineers (SPIE) Conf. Ser., ed. S. D'Odorico, 3355, 36

D’Andrea, C. B., Sako, M., Dilday, B., et al. 2010, ApJ, 708, 661

Dekker, H., Delabre, B., \& D'Odorico, S. 1986, in SPIE Conf. Ser. 627, ed. D. L. Crawford, 339

Ellis, R. S., Sullivan, M., Nugent, P. E., et al. 2008, ApJ, 674, 51 Filippenko, A. V. 1982, PASP, 94, 715

Foley, R. J., Filippenko, A. V., Aguilera, C., et al. 2008, ApJ, 684, 68 Foley, R. J., Matheson, T., Blondin, S., et al. 2009, AJ, 137, 3731 Frieman, J. A., Bassett, B., Becker, A., et al. 2008, AJ, 135, 338 Fukugita, M., Ichikawa, T., Gunn, J. E., et al. 1996, AJ, 111, 1748 Garavini, G., Folatelli, G., Goobar, A., et al. 2004, AJ, 128, 387

Garavini, G., Folatelli, G., Nobili, S., et al. 2007, A\&A, 470, 411 Garnavich, P. M., Bonanos, A. Z., Krisciunas, K., et al. 2004, ApJ, 613, 1120 Guy, J., Astier, P., Nobili, S., Regnault, N., \& Pain, R. 2005, A\&A, 443, 781

Guy, J., Astier, P., Baumont, S., et al. 2007, A\&A, 466, 11

Hicken, M., Challis, P., Jha, S., et al. 2009, ApJ, 700, 331

Holtzman, J. A., Marriner, J., Kessler, R., et al. 2008, AJ, 136, 2306

Hook, I. M., Howell, D. A., Aldering, G., et al. 2005, AJ, 130, 2788 Horne, K. 1986, PASP, 98, 609

Howell, D. A., Sullivan, M., Perrett, K., et al. 2005, ApJ, 634, 1190 Hsiao, E. Y., Conley, A., Howell, D. A., et al. 2007, ApJ, 663, 1187 Jha, S., Kirshner, R. P., Challis, P., et al. 2006, AJ, 131, 527

Kessler, R., Becker, A. C., Cinabro, D., et al. 2009, ApJS, 185, 32 Kinney, A. L., Calzetti, D., Bohlin, R. C., et al. 1996, ApJ, 467, 38 Lampeitl, H., Nichol, R. C., Seo, H., et al. 2010, MNRAS, 401, 2331

Matheson, T., Kirshner, R. P., Challis, P., et al. 2008, AJ, 135, 1598

Nobili, S., \& Goobar, A. 2008, A\&A, 487, 19

Nordin, J., Goobar, A., \& Jönsson, J. 2008, J. Cosmol. Astro-Part. Phys., 2, 8

Nordin, J., Östman, L., Goobar, A., et al. 2011, A\&A, 526, A119

Nugent, P., Kim, A., \& Perlmutter, S. 2002, PASP, 114, 803

O’Donnell, J. E. 1994, ApJ, 422, 158

Owens, J. C. 1967, Appl. Opt., 6, 51

Perlmutter, S., Aldering, G., Goldhaber, G., et al. 1999, ApJ, 517, 565

Riess, A. G., Filippenko, A. V., Challis, P., et al. 1998, AJ, 116, 1009

Sahu, D. K., Tanaka, M., Anupama, G. C., et al. 2008, ApJ, 680, 580

Sako, M., Bassett, B., Becker, A., et al. 2008, AJ, 135, 348

Schlegel, D. J., Finkbeiner, D. P., \& Davis, M. 1998, ApJ, 500, 525

Schroeder, D. J. 1987, Astronomical optics (San Diego, CA: Academic Press, Inc)

Sollerman, J., Mörtsell, E., Davis, T. M., et al. 2009, ApJ, 703, 1374

Stanishev, V. 2007, Astron. Nachr., 328, 948

Stanishev, V., Goobar, A., Benetti, S., et al. 2007, A\&A, 469, 645

Szokoly, G. P., Bergeron, J., Hasinger, G., et al. 2004, ApJS, 155, 271

Yip, C. W., Connolly, A. J., Szalay, A. S., et al. 2004, AJ, 128, 585

van Velzen, S., Farrar, G. R., Gezari, S., et al. 2010, ApJ, submitted [arXiv: 1009.1627]

York, D. G., Adelman, J., Anderson, Jr., J. E., et al. 2000, AJ, 120, 1579

Zheng, C., Romani, R. W., Sako, M., et al. 2008, AJ, 135, 1766 


\section{Appendix A: Tables}

Table A.1. Information about the observations.

\begin{tabular}{|c|c|c|c|c|c|c|c|}
\hline ID & SPID & $\mathrm{IAU}^{a}$ & Telescope & $\begin{array}{l}\text { Slit width } \\
(\operatorname{arcsec})\end{array}$ & $\begin{array}{l}\text { Exposure } \\
\text { time }(s)\end{array}$ & Airmass & $\begin{array}{l}\text { Seeing } \\
(\operatorname{arcsec})^{b}\end{array}$ \\
\hline 12778 & 692 & $2006 \mathrm{fs}$ & NTT & 1.0 & 1000 & 1.16 & 1.1 \\
\hline 12779 & 693 & $2006 \mathrm{fd}$ & NTT & 1.5 & 1000 & 1.27 & 1.2 \\
\hline 12781 & 680 & $2006 \mathrm{er}$ & NTT & 1.5 & 1800 & 1.14 & 1.9 \\
\hline 12782 & 681 & $2006 f \mathrm{f}$ & NTT & 1.5 & 1000 & 1.27 & 1.6 \\
\hline 12820 & 711 & $2006 f g$ & NTT & 1.5 & 600 & 1.41 & 1.8 \\
\hline 12842 & 682 & $2006 \mathrm{ez}$ & NTT & 1.5 & 800 & 1.14 & 1.5 \\
\hline 12843 & 727 & $2006 f a$ & NTT & 1.0 & 1000 & 1.15 & 0.8 \\
\hline 12844 & 684 & $2006 f e$ & NTT & 1.0 & 2000 & 1.21 & 1.6 \\
\hline 12853 & 685 & 2006еу & NTT & 1.5 & 1000 & 1.17 & 1.1 \\
\hline 12855 & 716 & $2006 \mathrm{fk}$ & NTT & 1.5 & 2000 & 1.25 & 2.0 \\
\hline 12856 & 695 & $2006 f l$ & NTT & 1.5 & 2197 & 1.38 & 1.5 \\
\hline 12860 & 688 & $2006 \mathrm{fc}$ & NTT & 1.5 & 1000 & 1.16 & 1.6 \\
\hline 12874 & 689 & $2006 \mathrm{fb}$ & NTT & 1.5 & 2400 & 1.20 & 2.5 \\
\hline 12898 & 712 & $2006 \mathrm{fw}$ & NTT & 1.5 & 600 & 1.35 & 1.5 \\
\hline 12907 & 714 & $2006 \mathrm{fv}$ & NTT & 1.5 & 600 & 1.21 & 1.6 \\
\hline 12927 & 690 & 2006fj & NTT & 1.5 & 1800 & 1.25 & 2.5 \\
\hline 12928 & 686 & $2006 \mathrm{ew}$ & NTT & 1.0 & 2000 & 1.20 & 1.2 \\
\hline 12930 & 687 & $2006 \mathrm{ex}$ & NTT & 1.5 & 1000 & 1.16 & 1.4 \\
\hline 12947 & 691 & & NTT & 1.5 & 1500 & 1.36 & 1.7 \\
\hline 12950 & 700 & $2006 f y$ & NTT & 1.0 & 600 & 1.19 & 1.1 \\
\hline 12950 & 1055 & $2006 f y$ & NTT & 1.0 & 600 & 1.37 & 1.1 \\
\hline 12978 & 701 & & NTT & 1.5 & 1800 & 1.59 & 3.0 \\
\hline 13005 & 702 & 2006fh & NTT & 1.5 & 2000 & 1.14 & 2.0 \\
\hline 13025 & 761 & $2006 f x$ & NTT & 1.0 & 1200 & 1.24 & 0.8 \\
\hline 13044 & 724 & $2006 \mathrm{fm}$ & NTT & 1.0 & 900 & 1.19 & 1.0 \\
\hline 13044 & 1062 & $2006 \mathrm{fm}$ & NTT & 1.0 & 1200 & 1.60 & 1.2 \\
\hline 13045 & 734 & 2006fn & NTT & 1.0 & 1000 & 1.41 & 1.7 \\
\hline 13046 & 726 & & NTT & 1.0 & 1500 & 1.46 & 1.4 \\
\hline 13070 & 736 & $2006 f u$ & NTT & 1.0 & 2000 & 1.19 & 0.9 \\
\hline 13072 & 723 & 2006fi & NTT & 1.0 & 1500 & 1.30 & 1.7 \\
\hline 13135 & 739 & $2006 \mathrm{fz}$ & NTT & 1.0 & 1200 & 1.19 & 1.7 \\
\hline 13135 & 998 & $2006 f z$ & NTT & 1.0 & 600 & 1.26 & 1.2 \\
\hline 13174 & 766 & 2006ga & NTT & 1.5 & 1000 & 1.48 & 1.6 \\
\hline 13195 & 764 & $2006 \mathrm{fo}$ & NTT & 1.0 & 300 & 2.04 & 1.7 \\
\hline 13195 & 983 & $2006 f o$ & NTT & 1.0 & 900 & 1.66 & 1.7 \\
\hline 13195 & 1458 & 2006 fo & NTT & 1.0 & 900 & 2.00 & 0.8 \\
\hline 13355 & 1003 & $2006 \mathrm{kh}$ & NTT & 1.0 & 900 & 1.47 & 1.4 \\
\hline 13376 & 1002 & $2006 \mathrm{gq}$ & NTT & 1.0 & 1200 & 1.36 & 1.4 \\
\hline 13376 & 1106 & $2006 \mathrm{gq}$ & NTT & 1.0 & 1800 & 1.66 & 1.4 \\
\hline 13577 & 1000 & $2006 \mathrm{~kg}$ & NTT & 1.0 & 900 & 1.62 & 1.7 \\
\hline 13796 & 1058 & 2006hl & NTT & 1.0 & 900 & 1.51 & 1.3 \\
\hline 13894 & 1039 & 2006jh & NTT & 1.0 & 1800 & 1.35 & 1.1 \\
\hline 14157 & 1040 & $2006 \mathrm{kj}$ & NTT & 1.0 & 1800 & 1.26 & 1.0 \\
\hline 14279 & 1459 & 2006hx & NTT & 1.0 & 900 & 1.87 & 0.9 \\
\hline 14318 & 1594 & 2006py & NTT & 1.0 & 900 & 2.47 & 1.0 \\
\hline 14318 & 1653 & 2006py & NTT & 1.0 & 600 & 1.99 & 1.6 \\
\hline 14318 & 1713 & 2006py & NTT & 1.5 & 1800 & 1.77 & 2.3 \\
\hline 14437 & 1061 & 2006hy & NTT & 1.0 & 900 & 1.43 & 1.2 \\
\hline 14450 & 991 & $2006 \mathrm{kn}$ & NTT & 1.0 & 1800 & 1.21 & 1.0 \\
\hline 14451 & 989 & $2006 \mathrm{ji}$ & NTT & 1.0 & 1200 & 1.42 & 1.5 \\
\hline 14492 & 1001 & 2006jo & NTT & 1.0 & 1800 & 1.31 & 1.4 \\
\hline 14598 & 987 & & NTT & 1.0 & 900 & 1.20 & 1.3 \\
\hline 14599 & 988 & 2006jl & NTT & 1.0 & 600 & 1.16 & 1.1 \\
\hline 14782 & 990 & 2006jp & NTT & 1.0 & 900 & 1.25 & 1.8 \\
\hline 14846 & 1014 & 2006jn & NTT & 1.0 & 1200 & 1.32 & 1.2 \\
\hline 14871 & 1008 & $2006 \mathrm{jq}$ & NTT & 1.0 & 600 & 1.17 & 1.8 \\
\hline 14979 & 1009 & $2006 \mathrm{jr}$ & NTT & 1.5 & 900 & 1.21 & 1.9 \\
\hline 14984 & 1027 & $2006 \mathrm{js}$ & NTT & 1.0 & 1800 & 1.18 & 1.1 \\
\hline 15031 & 985 & 2006iw & NTT & 1.0 & 450 & 1.17 & 1.6 \\
\hline 15129 & 1015 & $2006 \mathrm{kq}$ & NTT & 1.0 & 1800 & 1.24 & 1.0 \\
\hline 15132 & 1012 & $2006 \mathrm{jt}$ & NTT & 1.0 & 900 & 1.20 & 1.8 \\
\hline 15136 & 1022 & 2006ju & NTT & 1.0 & 900 & 1.33 & 1.2 \\
\hline
\end{tabular}


L. Östman et al.: NTT and NOT spectroscopy of SDSS-II supernovae

Table A.1. continued.

\begin{tabular}{|c|c|c|c|c|c|c|c|}
\hline ID & SPID & $\mathrm{IAU}^{a}$ & Telescope & $\begin{array}{l}\text { Slit width } \\
(\operatorname{arcsec})\end{array}$ & $\begin{array}{l}\text { Exposure } \\
\text { time (s) }\end{array}$ & Airmass & $\begin{array}{l}\text { Seeing } \\
(\operatorname{arcsec})^{b}\end{array}$ \\
\hline 15153 & 1046 & & NTT & 1.0 & 900 & 1.26 & 1.7 \\
\hline 15161 & 1010 & $2006 \mathrm{jw}$ & NTT & 1.0 & 1800 & 1.48 & 1.3 \\
\hline 15171 & 1045 & $2006 \mathrm{~kb}$ & NTT & 1.0 & 1800 & 1.26 & 1.4 \\
\hline 15203 & 1026 & 2006jy & NTT & 1.0 & 1800 & 1.48 & 1.8 \\
\hline 15207 & 1038 & & NTT & 1.0 & 1200 & 1.51 & 1.6 \\
\hline 15210 & 1005 & & NTT & 1.0 & 1800 & 1.23 & 1.3 \\
\hline 15210 & 1052 & & NTT & 1.0 & 1800 & 1.30 & 1.1 \\
\hline 15222 & 1004 & 2006jz & NTT & 1.0 & 1800 & 1.53 & 1.3 \\
\hline 15234 & 1043 & $2006 \mathrm{kd}$ & NTT & 1.0 & 900 & 1.69 & 2.3 \\
\hline 15259 & 1051 & $2006 \mathrm{kc}$ & NTT & 1.0 & 1800 & 1.30 & 1.5 \\
\hline 15287 & 1057 & $2006 \mathrm{kt}$ & NTT & 1.0 & 1800 & 1.29 & 1.0 \\
\hline 15320 & 1098 & $2006 \mathrm{kv}$ & NTT & 1.0 & 1200 & 1.50 & 1.6 \\
\hline 15339 & 1107 & 2006ns & NTT & 1.0 & 1800 & 1.48 & 1.1 \\
\hline 15354 & 1110 & 2006lp & NTT & 1.0 & 1800 & 1.54 & 1.6 \\
\hline 15475 & 1464 & $20061 \mathrm{c}$ & NTT & 1.0 & 900 & 2.26 & 1.3 \\
\hline 15557 & 1532 & $2006 \mathrm{oz}$ & NOT & 1.3 & 2000 & 1.30 & 1.0 \\
\hline 16021 & 1355 & $2006 \mathrm{nc}$ & NOT & 1.3 & 1800 & 1.14 & 1.4 \\
\hline 16069 & 1358 & 2006nd & NOT & 1.3 & 2000 & 2.67 & 1.9 \\
\hline 16069 & 1467 & 2006nd & NTT & 1.0 & 1200 & 1.67 & 1.0 \\
\hline 16069 & 1651 & 2006nd & NTT & 1.5 & 1800 & 1.78 & 2.0 \\
\hline 16087 & 1455 & 2006pc & NTT & 1.0 & 1800 & 1.67 & 0.8 \\
\hline 16163 & 1678 & & NTT & 1.5 & 1800 & 1.57 & 1.9 \\
\hline 16165 & 1326 & 2006nw & NOT & 1.3 & 2100 & 1.60 & 1.5 \\
\hline 16179 & 1323 & $2006 n x$ & NOT & 1.3 & 1800 & 2.08 & 1.4 \\
\hline 16179 & 1469 & $2006 n x$ & NTT & 1.0 & 1200 & 1.83 & 0.6 \\
\hline 16179 & 1570 & $2006 n x$ & NTT & 1.0 & 1200 & 2.21 & 0.6 \\
\hline 16192 & 1322 & 2006ny & NOT & 1.3 & 1400 & 1.75 & 1.3 \\
\hline 16192 & 1496 & 2006 ny & NTT & 1.0 & 1800 & 1.73 & 1.6 \\
\hline 16204 & 1500 & & NTT & 1.0 & 900 & 2.02 & 1.5 \\
\hline 16206 & 1501 & 2006pe & NTT & 1.0 & 1200 & 1.51 & 1.0 \\
\hline 16215 & 1456 & 2006ne & NTT & 1.0 & 900 & 2.02 & 0.9 \\
\hline 16215 & 1630 & 2006ne & NTT & 1.0 & 1800 & 1.83 & 1.4 \\
\hline 16241 & 1470 & & NTT & 1.0 & 1800 & 1.75 & 1.0 \\
\hline 16280 & 1471 & 2006nz & NTT & 1.0 & 900 & 1.50 & 0.6 \\
\hline 16280 & 1564 & 2006nz & NTT & 1.0 & 1200 & 1.84 & 1.8 \\
\hline 16287 & 1449 & 2006np & NTT & 1.0 & 900 & 1.81 & 0.6 \\
\hline 16287 & 1569 & 2006np & NTT & 1.0 & 900 & 2.26 & 0.7 \\
\hline 16287 & 1650 & 2006np & NTT & 1.0 & 1800 & 1.70 & 1.7 \\
\hline 16302 & 1473 & & NTT & 1.0 & 1800 & 2.10 & 1.9 \\
\hline 16314 & 1335 & 2006 a & NOT & 1.3 & 1200 & 1.31 & 1.7 \\
\hline 16314 & 1475 & 2006oa & NOT & 1.3 & 1000 & 1.18 & 0.9 \\
\hline 16333 & 1367 & 2006on & NOT & 1.3 & 2000 & 1.48 & 1.3 \\
\hline 16352 & 1478 & $2006 \mathrm{pk}$ & NTT & 1.0 & 1800 & 1.82 & 1.1 \\
\hline 16391 & 1452 & & NTT & 1.0 & 1200 & 1.69 & 0.9 \\
\hline 16391 & 1565 & & NTT & 1.0 & 1800 & 1.81 & 0.9 \\
\hline 16392 & 1365 & $2006 \mathrm{ob}$ & NOT & 1.3 & 1100 & 3.06 & 1.9 \\
\hline 16392 & 1448 & $2006 \mathrm{ob}$ & NTT & 1.0 & 900 & 1.89 & 0.8 \\
\hline 16392 & 1566 & $2006 \mathrm{ob}$ & NTT & 1.0 & 1200 & 2.18 & 2.3 \\
\hline 16392 & 1682 & $2006 \mathrm{ob}$ & NTT & 1.5 & 1800 & 1.43 & 1.8 \\
\hline 16402 & 1505 & $2006 s v$ & NTT & 1.0 & 1200 & 2.38 & 0.9 \\
\hline 16473 & 1520 & 2006pl & NTT & 1.0 & 1800 & 1.85 & 1.0 \\
\hline 16541 & 1485 & $2006 \mathrm{pn}$ & NOT & 1.3 & 2400 & 1.76 & 1.4 \\
\hline 16578 & 1516 & 2006po & NTT & 1.0 & 1800 & 2.19 & 1.2 \\
\hline 16619 & 1519 & $2006 \mathrm{ps}$ & NTT & 1.0 & 900 & 2.05 & 0.9 \\
\hline 16619 & 1528 & 2006ps & NOT & 1.3 & 1800 & 1.30 & 1.4 \\
\hline 16637 & 1514 & & NTT & 1.0 & 1800 & 1.47 & 0.7 \\
\hline 16641 & 1518 & 2006pr & NTT & 1.0 & 900 & 1.88 & 0.8 \\
\hline 16641 & 1530 & $2006 \mathrm{pr}$ & NOT & 1.3 & 1800 & 1.17 & 1.3 \\
\hline 16641 & 1649 & $2006 \mathrm{pr}$ & NTT & 1.0 & 1200 & 2.00 & 1.5 \\
\hline 16668 & 1561 & $2006 \mathrm{pu}$ & NOT & 1.3 & 2000 & 1.33 & 1.3 \\
\hline 16692 & 1489 & 2006op & NTT & 1.0 & 900 & 1.85 & 1.0 \\
\hline 16737 & 1599 & $2006 q c$ & NTT & 1.0 & 1200 & 2.29 & 0.7 \\
\hline 16741 & 1523 & & NOT & 1.3 & 600 & 1.23 & 1.1 \\
\hline 16748 & 1574 & 2006sx & NTT & 1.0 & 1800 & 1.95 & 0.8 \\
\hline 16774 & 1606 & 2006sy & NTT & 1.0 & 1800 & 2.04 & 0.9 \\
\hline 16778 & 1542 & & NTT & 1.0 & 1800 & 1.83 & 0.6 \\
\hline
\end{tabular}


Table A.1. continued.

\begin{tabular}{|c|c|c|c|c|c|c|c|}
\hline ID & SPID & $\mathrm{IAU}^{a}$ & Telescope & $\begin{array}{l}\text { Slit width } \\
(\operatorname{arcsec})\end{array}$ & $\begin{array}{l}\text { Exposure } \\
\text { time (s) }\end{array}$ & Airmass & $\begin{array}{l}\text { Seeing } \\
(\operatorname{arcsec})^{b}\end{array}$ \\
\hline 16778 & 1568 & & NTT & 1.0 & 1800 & 1.66 & 0.8 \\
\hline 16793 & 1603 & 2006qg & NTT & 1.0 & 1200 & 2.32 & 0.9 \\
\hline 16838 & 1522 & & NOT & 1.3 & 2500 & 1.45 & 1.1 \\
\hline 16857 & 1538 & & NTT & 1.0 & 1200 & 1.93 & 0.6 \\
\hline 16867 & 1541 & & NTT & 1.0 & 1800 & 1.73 & 1.1 \\
\hline 16872 & 1539 & 2006qh & NTT & 1.0 & 1200 & 2.00 & 0.6 \\
\hline 16956 & 1562 & 2006qj & NTT & 1.0 & 1500 & 2.34 & 0.9 \\
\hline 16979 & 1597 & & NTT & 1.0 & 1800 & 2.07 & 1.0 \\
\hline 16988 & 1595 & $2006 \mathrm{qk}$ & NTT & 1.0 & 1200 & 2.27 & 0.8 \\
\hline 16988 & 1652 & $2006 \mathrm{qk}$ & NTT & 1.0 & 1800 & 1.99 & 1.5 \\
\hline 17117 & 1679 & 2006qm & NTT & 1.0 & 1800 & 1.96 & 1.4 \\
\hline 17135 & 1648 & 2006rz & NTT & 1.0 & 1800 & 1.92 & 1.8 \\
\hline 17167 & 2250 & $2007 \mathrm{mr}$ & NTT & 1.0 & 1800 & 1.29 & 1.2 \\
\hline 17170 & 1879 & & NTT & 1.0 & 1800 & 1.22 & 1.4 \\
\hline 17176 & 1812 & 2007ie & NTT & 1.5 & 1200 & 1.16 & 1.7 \\
\hline 17200 & 1796 & $2007 \mathrm{ja}$ & NTT & 1.5 & 1800 & 1.32 & 1.7 \\
\hline 17206 & 1788 & & NTT & 1.5 & 1800 & 1.27 & 1.4 \\
\hline 17218 & 1794 & 2007jp & NOT & 1.0 & 2000 & 1.14 & 0.8 \\
\hline 17220 & 1791 & $2007 \mathrm{ji}$ & NTT & 1.5 & 1200 & 1.26 & 1.8 \\
\hline 17223 & 1793 & $2007 \mathrm{jj}$ & NTT & 1.5 & 1800 & 1.21 & 2.3 \\
\hline 17237 & 1830 & $2007 \mathrm{jc}$ & NTT & 1.5 & 1200 & 1.15 & 2.3 \\
\hline 17245 & 2234 & & NTT & 1.0 & 1800 & 1.20 & 0.9 \\
\hline 17247 & 1799 & & NTT & 1.0 & 1800 & 1.18 & 1.1 \\
\hline 17253 & 1898 & 2007 jq & NOT & 1.0 & 2000 & 1.16 & 0.8 \\
\hline 17254 & 1813 & $2007 \mathrm{ii}$ & NTT & 1.5 & 1800 & 1.21 & 1.8 \\
\hline 17332 & 1899 & $2007 \mathrm{jk}$ & NOT & 1.0 & 1400 & 1.20 & 0.9 \\
\hline 17351 & 1769 & 2007 jy & NTT & 1.0 & 1800 & 1.17 & 1.1 \\
\hline 17366 & 1782 & $2007 \mathrm{hz}$ & NTT & 1.0 & 1200 & 1.14 & 1.4 \\
\hline 17389 & 1811 & 2007ih & NTT & 1.0 & 1800 & 1.14 & 1.6 \\
\hline 17391 & 1872 & 2007jo & NTT & 1.5 & 1800 & 1.18 & 1.5 \\
\hline 17422 & 1785 & & NTT & 1.0 & 1800 & 1.18 & 0.9 \\
\hline 17435 & 1902 & $2007 \mathrm{ka}$ & NOT & 1.0 & 2000 & 1.25 & 1.0 \\
\hline 17436 & 1790 & & NTT & 1.5 & 1800 & 1.34 & 1.9 \\
\hline 17464 & 1853 & $2007 \mathrm{jb}$ & NTT & 1.5 & 2400 & 1.47 & 2.5 \\
\hline 17486 & 1854 & & NTT & 1.0 & 1800 & 1.14 & 1.9 \\
\hline 17497 & 1837 & $2007 \mathrm{jt}$ & NOT & 1.0 & 2000 & 1.17 & 0.8 \\
\hline 17500 & 2249 & 2007lf & NTT & 1.0 & 1800 & 1.19 & 1.0 \\
\hline 17535 & 1838 & & NOT & 1.0 & 2000 & 1.16 & 0.8 \\
\hline 17548 & 1825 & $2007 \mathrm{~ms}$ & NTT & 1.0 & 1200 & 1.15 & 1.1 \\
\hline 17548 & 2231 & $2007 \mathrm{~ms}$ & NTT & 1.0 & 1200 & 1.25 & 0.9 \\
\hline 17548 & 2293 & $2007 \mathrm{~ms}$ & NTT & 1.5 & 1800 & 1.24 & 2.0 \\
\hline 17552 & 1789 & $2007 j 1$ & NTT & 1.5 & 1800 & 1.29 & 1.7 \\
\hline 17568 & 1810 & $2007 \mathrm{~kb}$ & NTT & 1.0 & 1800 & 1.17 & 1.2 \\
\hline 17605 & 1809 & $2007 \mathrm{js}$ & NTT & 1.5 & 1200 & 1.33 & 1.8 \\
\hline 17627 & 1841 & $2007 j f$ & NOT & 1.0 & 2000 & 1.16 & 0.7 \\
\hline 17629 & 1851 & $2007 \mathrm{jw}$ & NTT & 1.5 & 1800 & 1.29 & 2.0 \\
\hline 17647 & 1875 & & NTT & 1.0 & 1800 & 1.42 & 1.4 \\
\hline 17703 & 1881 & & NTT & 1.5 & 1800 & 1.25 & 1.9 \\
\hline 17745 & 2161 & $2007 \mathrm{ju}$ & NTT & 1.5 & 1800 & 1.32 & 2.8 \\
\hline 17746 & 1873 & $2007 \mathrm{jv}$ & NTT & 1.5 & 1800 & 1.16 & 1.7 \\
\hline 17784 & 1842 & $2007 \mathrm{jg}$ & NOT & 1.0 & 700 & 1.19 & 0.7 \\
\hline 17790 & 1887 & $2007 \mathrm{jx}$ & NOT & 1.0 & 2000 & 1.28 & 1.0 \\
\hline 17794 & 1906 & & NTT & 1.0 & 1800 & 1.16 & 1.1 \\
\hline 17811 & 1816 & 2007ix & NTT & 1.5 & 1800 & 1.14 & 2.2 \\
\hline 17814 & 1901 & & NTT & 1.0 & 1800 & 1.14 & 0.9 \\
\hline 17825 & 1819 & 2007je & NTT & 1.5 & 1800 & 1.25 & 2.7 \\
\hline 17854 & 2230 & & NTT & 1.0 & 1800 & 1.48 & 0.8 \\
\hline 17875 & 1817 & $2007 \mathrm{jz}$ & NTT & 1.0 & 1800 & 1.23 & 1.3 \\
\hline 17880 & 1843 & $2007 \mathrm{jd}$ & NOT & 1.0 & 900 & 1.20 & 0.7 \\
\hline 17880 & 1957 & $2007 \mathrm{jd}$ & NTT & 1.5 & 1800 & 1.26 & 2.3 \\
\hline 17880 & 2194 & $2007 \mathrm{jd}$ & NTT & 1.5 & 1800 & 1.51 & 2.0 \\
\hline 17886 & 1844 & $2007 \mathrm{jh}$ & NOT & 1.0 & 600 & 1.13 & 0.9 \\
\hline 17924 & 1826 & & NTT & 1.5 & 1200 & 1.20 & 2.2 \\
\hline 17973 & 1926 & & NOT & 1.0 & 900 & 1.14 & 0.9 \\
\hline 17973 & 1942 & & NTT & 1.0 & 1200 & 1.19 & 0.9 \\
\hline 18109 & 1940 & $2007 \mathrm{kw}$ & NTT & 1.0 & 1200 & 1.19 & 1.3 \\
\hline
\end{tabular}


L. Östman et al.: NTT and NOT spectroscopy of SDSS-II supernovae

Table A.1. continued.

\begin{tabular}{|c|c|c|c|c|c|c|c|}
\hline ID & SPID & $\mathrm{IAU}^{a}$ & Telescope & $\begin{array}{l}\text { Slit width } \\
(\operatorname{arcsec})\end{array}$ & $\begin{array}{l}\text { Exposure } \\
\text { time (s) }\end{array}$ & Airmass & $\begin{array}{l}\text { Seeing } \\
(\operatorname{arcsec})^{b}\end{array}$ \\
\hline 18325 & 2277 & $2007 \mathrm{mv}$ & NTT & 1.0 & 1800 & 1.21 & 0.9 \\
\hline 18457 & 2285 & 200711 & NTT & 1.5 & 1800 & 1.38 & 2.4 \\
\hline 18466 & 2270 & $2007 \mathrm{~lm}$ & NTT & 1.0 & 1800 & 1.34 & 1.0 \\
\hline 18590 & 2248 & $2007 \mathrm{nw}$ & NTT & 1.0 & 1800 & 1.35 & 0.8 \\
\hline 18596 & 2227 & 2007ld & NTT & 1.5 & 1500 & 1.31 & 2.1 \\
\hline 18647 & 2271 & & NTT & 1.0 & 1800 & 1.22 & 0.8 \\
\hline 18697 & 2171 & $2007 \mathrm{ma}$ & NTT & 1.5 & 1800 & 1.33 & 2.6 \\
\hline 18768 & 2135 & 2007lh & NTT & 1.0 & 1800 & 1.24 & 1.0 \\
\hline 18787 & 2150 & $2007 \mathrm{mf}$ & NTT & 1.0 & 1800 & 1.34 & 0.8 \\
\hline 18804 & 2148 & $2007 \mathrm{me}$ & NTT & 1.0 & 1500 & 1.22 & 1.0 \\
\hline 18903 & 2247 & $20071 r$ & NTT & 1.0 & 1800 & 1.18 & 1.0 \\
\hline 18965 & 2279 & 2007ne & NTT & 1.0 & 1800 & 1.29 & 0.9 \\
\hline 19003 & 2235 & $2007 \mathrm{mp}$ & NTT & 1.0 & 1800 & 1.42 & 0.8 \\
\hline 19003 & 2290 & $2007 \mathrm{mp}$ & NTT & 1.0 & 1800 & 1.35 & 1.9 \\
\hline 19008 & 2284 & $2007 \mathrm{mz}$ & NTT & 1.0 & 1800 & 1.15 & 0.8 \\
\hline 19023 & 2236 & 2007ls & NTT & 1.0 & 2000 & 1.21 & 1.1 \\
\hline 19051 & 2297 & $2007 \mathrm{nb}$ & NTT & 1.0 & 1800 & 1.18 & 0.8 \\
\hline 19101 & 2268 & $2007 \mathrm{ml}$ & NTT & 1.0 & 543 & 1.29 & 1.2 \\
\hline 19149 & 2275 & $2007 \mathrm{ni}$ & NTT & 1.0 & 1800 & 1.30 & 1.1 \\
\hline 19155 & 2252 & $2007 \mathrm{mn}$ & NTT & 1.0 & 900 & 1.48 & 0.9 \\
\hline 19155 & 2607 & $2007 \mathrm{mn}$ & NOT & 1.3 & 1500 & 1.16 & 0.9 \\
\hline 19155 & 2720 & $2007 \mathrm{mn}$ & NTT & 1.0 & 1200 & 1.65 & 1.7 \\
\hline 19221 & 2274 & & NTT & 1.0 & 1800 & 1.19 & 1.0 \\
\hline 19222 & 2299 & 2007nl & NTT & 1.0 & 1800 & 1.15 & 0.7 \\
\hline 19230 & 2282 & $2007 \mathrm{mo}$ & NTT & 1.0 & 1800 & 1.24 & 1.2 \\
\hline 19282 & 2280 & $2007 \mathrm{mk}$ & NTT & 1.0 & 1800 & 1.19 & 1.1 \\
\hline 19323 & 2296 & $2007 \mathrm{nc}$ & NTT & 1.0 & 1800 & 1.20 & 0.8 \\
\hline 19341 & 2298 & $2007 \mathrm{nf}$ & NTT & 1.0 & 1800 & 1.39 & 1.1 \\
\hline 19353 & 2281 & $2007 \mathrm{nj}$ & NTT & 1.0 & 1800 & 1.31 & 1.2 \\
\hline 19381 & 2283 & $2007 \mathrm{nk}$ & NTT & 1.0 & 1800 & 1.36 & 1.2 \\
\hline 19899 & 2550 & $2007 \mathrm{pu}$ & NOT & 1.3 & 1800 & 1.14 & 0.9 \\
\hline 19913 & 2585 & $2007 \mathrm{qf}$ & NTT & 1.0 & 1800 & 1.45 & 0.9 \\
\hline 19953 & 2602 & $2007 \mathrm{pf}$ & NOT & 1.3 & 1800 & 1.13 & 0.9 \\
\hline 19968 & 2549 & $2007 \mathrm{ol}$ & NOT & 1.3 & 1200 & 1.30 & 1.0 \\
\hline 20039 & 2584 & 2007qh & NTT & 1.0 & 1800 & 1.44 & 1.5 \\
\hline 20040 & 2612 & $2007 \mathrm{rf}$ & NTT & 1.0 & 1800 & 1.76 & 0.8 \\
\hline 20052 & 2537 & & NOT & 1.3 & 1800 & 1.13 & 0.8 \\
\hline 20052 & 2538 & & NTT & 1.0 & 1200 & 1.80 & 1.5 \\
\hline 20088 & 2546 & & NTT & 1.0 & 1800 & 1.59 & 1.0 \\
\hline 20097 & 2587 & 2007rd & NTT & 1.0 & 1800 & 2.04 & 1.1 \\
\hline 20142 & 2586 & $2007 \mathrm{qg}$ & NTT & 1.0 & 3600 & 1.60 & 0.9 \\
\hline 20144 & 2541 & $2007 \mathrm{ql}$ & NTT & 1.0 & 1800 & 2.19 & 1.0 \\
\hline 20227 & 2568 & 2007qi & NTT & 1.0 & 1800 & 1.39 & 1.2 \\
\hline 20345 & 2567 & $2007 q p$ & NTT & 1.0 & 1800 & 1.88 & 1.1 \\
\hline 20364 & 2581 & $2007 \mathrm{qo}$ & NTT & 1.0 & 1800 & 2.33 & 1.0 \\
\hline 20376 & 2582 & 2007re & NTT & 1.0 & 1800 & 1.68 & 0.8 \\
\hline 20388 & 2611 & & NTT & 1.0 & 1800 & 1.64 & 1.1 \\
\hline 20430 & 2543 & 2007qj & NTT & 1.0 & 1800 & 2.03 & 0.9 \\
\hline 20474 & 2563 & $2007 \mathrm{rg}$ & NTT & 1.0 & 1800 & 1.62 & 0.6 \\
\hline 20474 & 2714 & $2007 \mathrm{rg}$ & NTT & 1.5 & 1800 & 2.73 & 1.5 \\
\hline 20474 & 3003 & $2007 \mathrm{rg}$ & NTT & 1.5 & 1800 & 3.49 & 2.6 \\
\hline 20530 & 2547 & & NTT & 1.0 & 1200 & 1.50 & 1.3 \\
\hline 20530 & 2571 & & NTT & 1.0 & 1800 & 1.38 & 1.0 \\
\hline 20575 & 2540 & 2007rh & NOT & 1.3 & 1800 & 1.15 & 1.2 \\
\hline 20575 & 3005 & 2007rh & NOT & 1.3 & 1800 & 1.40 & 1.4 \\
\hline 20625 & 2551 & $2007 \mathrm{px}$ & NOT & 1.3 & 1800 & 1.14 & 0.9 \\
\hline 20625 & 2604 & $2007 \mathrm{px}$ & NOT & 1.3 & 1800 & 1.14 & 0.9 \\
\hline 20677 & 2536 & $2007 q x$ & NTT & 1.0 & 1800 & 1.64 & 1.8 \\
\hline 20678 & 2610 & & NTT & 1.0 & 1800 & 2.47 & 1.4 \\
\hline 20687 & 2596 & 2007ri & NTT & 1.0 & 1800 & 1.89 & 1.3 \\
\hline 20687 & 2597 & 2007ri & NOT & 1.3 & 3600 & 1.14 & 1.3 \\
\hline 20718 & 2577 & 2007rj & NTT & 1.0 & 1300 & 3.15 & 1.0 \\
\hline 20718 & 2593 & 2007rj & NTT & 1.0 & 1800 & 1.93 & 1.1 \\
\hline 20764 & 2594 & 2007ro & NOT & 1.3 & 1800 & 1.15 & 1.3 \\
\hline 20834 & 2598 & $2007 \mathrm{rr}$ & NTT & 1.0 & 1800 & 2.05 & 1.2 \\
\hline 20862 & 2600 & 2007rn & NTT & 1.0 & 1800 & 1.49 & 1.1 \\
\hline
\end{tabular}


Table A.1. continued.

\begin{tabular}{|c|c|c|c|c|c|c|c|}
\hline ID & SPID & $\mathrm{IAU}^{a}$ & Telescope & $\begin{array}{l}\text { Slit width } \\
(\operatorname{arcsec})\end{array}$ & $\begin{array}{l}\text { Exposure } \\
\text { time }(s)\end{array}$ & Airmass & $\begin{array}{l}\text { Seeing } \\
(\operatorname{arcsec})^{b}\end{array}$ \\
\hline 20909 & 2580 & & NTT & 1.0 & 1800 & 2.38 & 1.2 \\
\hline 20978 & 2609 & 2007rl & NTT & 1.0 & 1800 & 2.16 & 1.0 \\
\hline 21006 & 2566 & $2007 \mathrm{qs}$ & NTT & 1.0 & 1800 & 1.66 & 0.9 \\
\hline 21033 & 2565 & 2007qy & NTT & 1.0 & 1800 & 1.61 & 0.8 \\
\hline 21034 & 2719 & 2007qa & NTT & 1.0 & 1200 & 1.53 & 1.1 \\
\hline 21034 & 2733 & $2007 q a$ & NTT & 1.5 & 1800 & 1.90 & 2.0 \\
\hline 21042 & 2564 & $2007 \mathrm{qz}$ & NTT & 1.0 & 1800 & 1.61 & 1.1 \\
\hline 21058 & 2579 & & NTT & 1.0 & 1800 & 1.62 & 0.7 \\
\hline 21058 & 2595 & & NOT & 1.3 & 1800 & 1.18 & 1.0 \\
\hline 21062 & 2613 & 2007rp & NTT & 1.0 & 1800 & 2.00 & 1.3 \\
\hline 21064 & 2532 & $2007 q b$ & NOT & 1.3 & 1800 & 1.28 & 1.2 \\
\hline 21064 & 2533 & $2007 q b$ & NTT & 1.0 & 1200 & 1.57 & 0.9 \\
\hline 21362 & 2636 & $2007 \mathrm{sd}$ & NOT & 1.3 & 2400 & 1.13 & 0.7 \\
\hline 21362 & 2697 & 2007sd & NTT & 1.0 & 1800 & 1.76 & 1.1 \\
\hline 21422 & 2599 & $2007 \mathrm{rq}$ & NTT & 1.0 & 1800 & 1.73 & 1.0 \\
\hline 21502 & 2574 & 2007ra & NOT & 1.3 & 2076 & 1.13 & 0.9 \\
\hline 21502 & 2575 & 2007ra & NOT & 1.3 & 1800 & 1.13 & 1.4 \\
\hline 21502 & 2717 & 2007ra & NTT & 1.5 & 1200 & 1.83 & 1.9 \\
\hline 21596 & 2588 & & NOT & 1.3 & 1800 & 1.19 & 1.1 \\
\hline 21596 & 2589 & & NTT & 1.0 & 1800 & 1.57 & 0.7 \\
\hline 21669 & 2591 & $2007 \mathrm{rs}$ & NTT & 1.0 & 1800 & 1.43 & 0.9 \\
\hline 21669 & 2722 & 2007rs & NTT & 1.0 & 900 & 1.29 & 1.6 \\
\hline 21766 & 2638 & $2007 \mathrm{rc}$ & NOT & 1.3 & 2400 & 1.14 & 0.9 \\
\hline 21810 & 2724 & $2007 \mathrm{se}$ & NTT & 1.0 & 1200 & 2.82 & 1.8 \\
\hline 21814 & 2702 & $2007 \mathrm{sf}$ & NTT & 1.5 & 1200 & 2.27 & 2.1 \\
\hline 21839 & 2716 & $2007 \mathrm{sl}$ & NTT & 1.5 & 1200 & 2.27 & 2.0 \\
\hline 21861 & 2723 & $2007 \mathrm{sg}$ & NTT & 1.0 & 1200 & 2.19 & 1.9 \\
\hline 21898 & 2704 & $2007 \mathrm{sj}$ & NTT & 1.0 & 1200 & 2.07 & 1.3 \\
\hline 22182 & 2690 & $2007 \mathrm{sm}$ & NTT & 1.5 & 1800 & 2.52 & 2.3 \\
\hline 22284 & 2735 & $2007 \mathrm{sn}$ & NTT & 1.0 & 1200 & 2.49 & 2.6 \\
\hline
\end{tabular}

Notes. ${ }^{(a)}$ Most of the spectra in the table that lack IAU names are photometric SNe without reliable spectroscopic confirmation and unclassified objects. ${ }^{(b)}$ The FWHM seeing at $5000 \AA$ for the airmass at which the observations were conducted. 
Table A.2. Spectroscopic typing and redshift determination.

\begin{tabular}{|c|c|c|c|c|c|c|c|c|}
\hline ID & SPID & SDSS type $^{a}$ & $\begin{array}{l}\text { NTT/NOT } \\
\text { type }^{b}\end{array}$ & $\mathrm{LC}_{\text {epoch }}{ }^{c}$ & $\begin{array}{l}\text { SNID } \\
\text { epoch }^{d}\end{array}$ & $\operatorname{SDSS} z^{e}$ & $\operatorname{SNID} z$ & Notes $^{f}$ \\
\hline 12778 & 692 & - & - & - & - & $0.0992 \pm 0.0001$ & - & \\
\hline 12779 & 693 & Ia & Ia-norm & $23.0 \pm 0.8^{p}$ & $26 \pm 16$ & $0.0800 \pm 0.0001$ & $0.078 \pm 0.003$ & $\mathrm{~s}$ \\
\hline 12781 & 680 & Ia & Ia-norm & $10.9 \pm 0.2$ & $20 \pm 18$ & $0.0843 \pm 0.0002$ & $0.084 \pm 0.004$ & \\
\hline 12782 & 681 & II & II & - & - & $0.06787 \pm 0.00005$ & - & $\mathrm{v}$ \\
\hline 12820 & 711 & II & IIP & - & $18 \pm 66$ & $0.04458 \pm 0.00005$ & $0.046 \pm 0.005$ & \\
\hline 12842 & 682 & II & II & - & - & $0.0887 \pm 0.0005$ & - & $\mathrm{v}, \mathrm{zg}$ \\
\hline 12843 & 727 & Ia & Ia-norm & $10.2 \pm 0.1$ & $16 \pm 25$ & $0.1670 \pm 0.0001$ & $0.161 \pm 0.006$ & s \\
\hline 12844 & 684 & Ic & - & - & - & $0.07053 \pm 0.00009$ & - & \\
\hline 12853 & 685 & Ia & Ia-norm & $10.3 \pm 0.2$ & $11 \pm 52$ & $0.1694 \pm 0.0005$ & $0.171 \pm 0.006$ & zg \\
\hline 12855 & 716 & Ia & Ia-norm & $-2.6 \pm 0.2$ & $0 \pm 5$ & $0.172 \pm 0.005$ & $0.171 \pm 0.005$ & $\mathrm{~s}, \mathrm{zs}$ \\
\hline 12856 & 695 & Ia & Ia-norm & $-3.2 \pm 0.2$ & $-1 \pm 4$ & $0.1717 \pm 0.0001$ & $0.171 \pm 0.005$ & $\mathrm{~s}$ \\
\hline 12860 & 688 & Ia & Ia-norm & $-1.9 \pm 0.9$ & $-1 \pm 5$ & $0.1217 \pm 0.0005$ & $0.124 \pm 0.005$ & $\mathrm{~s}, \mathrm{zg}$ \\
\hline 12874 & 689 & - & - & - & - & $0.2449 \pm 0.0002$ & - & \\
\hline 12898 & 712 & Ia & Ia-norm & $-6.6 \pm 0.1$ & $-1 \pm 5$ & $0.0835 \pm 0.0005$ & $0.078 \pm 0.004$ & $\mathrm{~s}, \mathrm{zg}$ \\
\hline 12907 & 714 & Ia & Ia-norm & $-0.1 \pm 0.2$ & $0 \pm 5$ & $0.1318 \pm 0.0002$ & $0.124 \pm 0.005$ & s \\
\hline 12927 & 690 & Ia & Ia-norm & $2.6 \pm 0.8^{p}$ & $-1 \pm 4$ & $0.175 \pm 0.005$ & $0.172 \pm 0.005$ & $\mathrm{~s}, \mathrm{zs}$ \\
\hline 12928 & 686 & Ia & Ia-norm & $17 \pm 1^{p}$ & $20 \pm 24$ & $0.1397 \pm 0.0005$ & $0.141 \pm 0.004$ & $\mathrm{~s}, \mathrm{zg}$ \\
\hline 12930 & 687 & Ia & Ia-norm & $10.1 \pm 0.2$ & $12 \pm 12$ & $0.1475 \pm 0.0002$ & $0.140 \pm 0.005$ & \\
\hline 12947 & 691 & photo-Ia & - & $1.4 \pm 0.2$ & - & $0.1592 \pm 0.0005$ & - & $\mathrm{zg}$ \\
\hline 12950 & 700 & Ia & Ia-norm & $-4.43 \pm 0.04$ & $-1 \pm 4$ & $0.08268 \pm 0.00004$ & $0.085 \pm 0.004$ & $\mathrm{~s}$ \\
\hline 12950 & 1055 & Ia & Ia-norm & $22.41 \pm 0.04$ & $32 \pm 9$ & $0.08268 \pm 0.00004$ & $0.085 \pm 0.002$ & $\mathrm{~s}$ \\
\hline 12978 & 701 & photo-Ia & - & - & - & - & - & $\mathrm{v}$ \\
\hline 13005 & 702 & Ia & Ia-norm & $24 \pm 1^{p}$ & $31 \pm 11$ & $0.1273 \pm 0.0005$ & $0.127 \pm 0.003$ & $\mathrm{~s}, \mathrm{zg}$ \\
\hline 13025 & 761 & Ia & Ia-norm & $3.4 \pm 0.3$ & $2 \pm 5$ & $0.2239 \pm 0.0005$ & $0.228 \pm 0.006$ & $\mathrm{zg}$ \\
\hline 13044 & 724 & Ia & Ia-norm & $-8.20 \pm 0.06$ & $-1 \pm 4$ & $0.1257 \pm 0.0005$ & $0.126 \pm 0.004$ & $\mathrm{zg}$ \\
\hline 13044 & 1062 & Ia & Ia-norm & $20.22 \pm 0.06$ & $24 \pm 18$ & $0.1257 \pm 0.0005$ & $0.126 \pm 0.004$ & $\mathrm{~s}, \mathrm{zg}$ \\
\hline 13045 & 734 & Ia & Ia & $0.5 \pm 0.3$ & $1 \pm 5$ & $0.1808 \pm 0.0005$ & $0.183 \pm 0.006$ & $\mathrm{~s}, \mathrm{zg}$ \\
\hline 13046 & 726 & - & Gal & - & - & $0.1259 \pm 0.0005$ & $0.1245 \pm 0.0009$ & $\mathrm{zg}, \mathrm{f}$ \\
\hline 13070 & 736 & Ia & Ia & $6.9 \pm 0.2$ & $8 \pm 2$ & $0.19855 \pm 0.00009$ & $0.195 \pm 0.007$ & \\
\hline 13072 & 723 & Ia & Ia-norm & $0 \pm 2$ & $-1 \pm 5$ & $0.2306 \pm 0.0008$ & $0.234 \pm 0.005$ & $\mathrm{~s}$ \\
\hline 13135 & 739 & Ia & Ia-norm & $-7.74 \pm 0.07$ & $-1 \pm 5$ & $0.1047 \pm 0.0001$ & $0.098 \pm 0.005$ & \\
\hline 13135 & 998 & Ia & Ia-norm & $17.64 \pm 0.07$ & $32 \pm 12$ & $0.1047 \pm 0.0001$ & $0.101 \pm 0.002$ & \\
\hline 13174 & 766 & Ia? & - & $-3.8 \pm 0.1$ & - & $0.2361 \pm 0.0005$ & - & $\mathrm{v}, \mathrm{zg}$ \\
\hline 13195 & 764 & $\mathrm{Ib}$ & $\mathrm{Ib}$ & - & $6 \pm 16$ & $0.0207 \pm 0.0001$ & $0.013 \pm 0.006$ & \\
\hline 13195 & 983 & $\mathrm{Ib}$ & Ib-norm & - & $14 \pm 21$ & $0.0207 \pm 0.0001$ & $0.022 \pm 0.007$ & \\
\hline 13195 & 1458 & $\mathrm{Ib}$ & $\mathrm{Ib}$ & - & - & $0.0207 \pm 0.0001$ & - & $\mathrm{v}$ \\
\hline 13355 & 1003 & II & IIP & - & $35 \pm 105$ & $0.05969 \pm 0.00008$ & $0.060 \pm 0.004$ & \\
\hline 13376 & 1002 & II & IIP & - & $33 \pm 76$ & $0.0698 \pm 0.0001$ & $0.069 \pm 0.005$ & \\
\hline 13376 & 1106 & II & IIP & - & $34 \pm 83$ & $0.0698 \pm 0.0001$ & $0.069 \pm 0.004$ & \\
\hline 13577 & 1000 & AGN & Gal & - & - & $0.2309 \pm 0.0005$ & $0.2309 \pm 0.0006$ & $\mathrm{zg}$ \\
\hline 13796 & 1058 & Ia & Ia-norm & $13 \pm 3$ & $15 \pm 42$ & $0.1482 \pm 0.0005$ & $0.149 \pm 0.006$ & $\mathrm{zg}$ \\
\hline 13894 & 1039 & Ia & Ia-norm & $9.2 \pm 0.1$ & $8 \pm 5$ & $0.1249 \pm 0.0005$ & $0.127 \pm 0.006$ & $\mathrm{~s}, \mathrm{zg}$ \\
\hline 14157 & 1040 & Ia & Ia & $9.4 \pm 0.2$ & $9 \pm 5$ & $0.2115 \pm 0.0005$ & $0.210 \pm 0.005$ & $\mathrm{~s}, \mathrm{zg}$ \\
\hline 14279 & 1459 & Ia & NotSN & $41.30 \pm 0.03$ & - & $0.0454 \pm 0.0002$ & $0.0461 \pm 0.0009$ & $\mathrm{~s}$ \\
\hline 14318 & 1594 & Ia & Ia-norm & $-4.3 \pm 0.3^{p}$ & $-3 \pm 5$ & $0.0579 \pm 0.0002$ & $0.054 \pm 0.004$ & $\mathrm{~s}$ \\
\hline 14318 & 1653 & Ia & Ia & $11.7 \pm 0.3^{p}$ & $12 \pm 12$ & $0.0579 \pm 0.0002$ & $0.056 \pm 0.005$ & $\mathrm{~s}$ \\
\hline 14318 & 1713 & Ia & Ia & $13.6 \pm 0.3^{p}$ & $16 \pm 10$ & $0.0579 \pm 0.0002$ & $0.057 \pm 0.004$ & $\mathrm{~s}$ \\
\hline 14437 & 1061 & Ia & Ia & $14.0 \pm 0.1$ & $17 \pm 41$ & $0.1491 \pm 0.0005$ & $0.148 \pm 0.005$ & $\mathrm{~s}, \mathrm{zg}$ \\
\hline 14450 & 991 & II & IIP & - & $15 \pm 9$ & $0.1203 \pm 0.0001$ & $0.117 \pm 0.003$ & \\
\hline 14451 & 989 & Ia & Ia-norm & $9.5 \pm 0.1$ & $8 \pm 2$ & $0.1784 \pm 0.0005$ & $0.1813 \pm 0.0010$ & $\mathrm{~s}, \mathrm{zg}$ \\
\hline 14492 & 1001 & $\mathrm{Ib}$ & $\mathrm{Ib} / \mathrm{c}$ & - & - & $0.0767 \pm 0.0001$ & - & $\mathrm{v}$ \\
\hline 14598 & 987 & - & - & - & - & - & - & \\
\hline 14599 & 988 & II & IIP & - & $5 \pm 25$ & $0.0555 \pm 0.0005$ & $0.048 \pm 0.005$ & $\mathrm{zg}$ \\
\hline 14782 & 990 & Ia & Ia-norm & $1.7 \pm 0.1$ & $-1 \pm 5$ & $0.1604 \pm 0.0005$ & $0.165 \pm 0.005$ & $\mathrm{~s}, \mathrm{zg}$ \\
\hline 14846 & 1014 & Ia & Ia-norm & $-1.7 \pm 0.2$ & $-1 \pm 4$ & $0.2247 \pm 0.0005$ & $0.223 \pm 0.005$ & $\mathrm{~s}, \mathrm{zg}$ \\
\hline 14871 & 1008 & Ia & Ia-norm & $-4.2 \pm 0.1$ & $-1 \pm 5$ & $0.1276 \pm 0.0005$ & $0.125 \pm 0.005$ & $\mathrm{~s}, \mathrm{zg}$ \\
\hline 14979 & 1009 & $\mathrm{Ia}$ & Ia-norm & $-2.1 \pm 0.1$ & $-2 \pm 4$ & $0.1771 \pm 0.0005$ & $0.178 \pm 0.006$ & $\mathrm{zg}$ \\
\hline 14984 & 1027 & Ia & Ia-norm & $-1.2 \pm 0.2$ & $0 \pm 5$ & $0.1967 \pm 0.0005$ & $0.191 \pm 0.005$ & $\mathrm{~s}, \mathrm{zg}$ \\
\hline 15031 & 985 & II & IIP & - & $4 \pm 22$ & $0.03073 \pm 0.00009$ & $0.028 \pm 0.004$ & \\
\hline 15129 & 1015 & Ia & Ia-norm & $1.8 \pm 0.1$ & $0 \pm 4$ & $0.1985 \pm 0.0002$ & $0.201 \pm 0.005$ & $\mathrm{~s}$ \\
\hline 15132 & 1012 & Ia & Ia-norm & $-2.4 \pm 0.2$ & $0 \pm 4$ & $0.144 \pm 0.005$ & $0.151 \pm 0.005$ & zs \\
\hline 15136 & 1022 & Ia? & Ia? & $-0.3 \pm 0.1$ & - & $0.14869 \pm 0.00005$ & - & $\mathrm{v}$ \\
\hline 15153 & 1046 & photo-II & - & - & - & - & - & $\mathrm{v}$ \\
\hline 15161 & 1010 & Ia & Ia-norm & $-1.0 \pm 0.4$ & $-4 \pm 5$ & $0.2496 \pm 0.0002$ & $0.253 \pm 0.005$ & \\
\hline 15171 & 1045 & Ia & Ia-norm & $-5.7 \pm 0.1$ & $-1 \pm 4$ & $0.134 \pm 0.005$ & $0.139 \pm 0.005$ & zs \\
\hline
\end{tabular}


Table A.2. continued.

\begin{tabular}{|c|c|c|c|c|c|c|c|c|}
\hline ID & SPID & SDSS type $^{a}$ & $\begin{array}{l}\text { NTT/NOT } \\
\text { type }^{b}\end{array}$ & $\mathrm{LC}_{\text {epoch }}{ }^{c}$ & $\begin{array}{l}\text { SNID } \\
\text { epoch }^{d}\end{array}$ & $\operatorname{SDSS} z^{e}$ & SNID $z$ & Notes $^{f}$ \\
\hline 15203 & 1026 & Ia & Ia-norm & $-2.4 \pm 0.2$ & $-1 \pm 5$ & $0.2043 \pm 0.0005$ & $0.199 \pm 0.006$ & $\mathrm{~s}, \mathrm{zg}$ \\
\hline 15207 & 1038 & - & - & - & - & $0.2582 \pm 0.0005$ & - & $\mathrm{zg}$ \\
\hline 15210 & 1005 & photo-non-Ia & - & - & - & $0.1262 \pm 0.0005$ & - & $\mathrm{v}, \mathrm{zg}$ \\
\hline 15210 & 1052 & photo-non-Ia & - & - & - & $0.1262 \pm 0.0005$ & - & $z g, f$ \\
\hline 15222 & 1004 & Ia & Ia-norm & $-5.8 \pm 0.2$ & $3 \pm 7$ & $0.1994 \pm 0.0001$ & $0.1975 \pm 0.0010$ & f \\
\hline 15234 & 1043 & Ia & Ia-norm & $-7.8 \pm 0.2$ & $-2 \pm 5$ & $0.13634 \pm 0.00009$ & $0.134 \pm 0.002$ & $\mathrm{~s}$ \\
\hline 15259 & 1051 & Ia & Ia-norm & $-1.9 \pm 0.2$ & $0 \pm 5$ & $0.2100 \pm 0.0001$ & $0.213 \pm 0.007$ & \\
\hline 15287 & 1057 & Ia & Ia & $-3.4 \pm 0.3$ & $0 \pm 5$ & $0.254 \pm 0.005$ & $0.255 \pm 0.009$ & zs \\
\hline 15320 & 1098 & II & - & - & - & $0.062 \pm 0.005$ & - & $\mathrm{v}, \mathrm{zs}$ \\
\hline 15339 & 1107 & II & - & - & - & $0.1200 \pm 0.0001$ & - & \\
\hline 15354 & 1110 & Ia & Gal & $1.3 \pm 0.4$ & - & $0.2221 \pm 0.0005$ & $0.2209 \pm 0.0006$ & $z g, f$ \\
\hline 15475 & 1464 & Ic & $\mathrm{Ib} / \mathrm{c}$ & - & - & $0.0162 \pm 0.0002$ & - & $\mathrm{v}$ \\
\hline 15557 & 1532 & II & IIP & - & $2 \pm 3$ & $0.2860 \pm 0.0005$ & $0.301 \pm 0.002$ & $\mathrm{zg}, \mathrm{f}$ \\
\hline 16021 & 1355 & Ia & Ia-norm & $11.29 \pm 0.09$ & $9 \pm 4$ & $0.124 \pm 0.005$ & $0.130 \pm 0.005$ & $\mathrm{~s}, \mathrm{zs}$ \\
\hline 16069 & 1358 & Ia & Ia & $4.3 \pm 0.2$ & - & $0.1288 \pm 0.0001$ & - & $\mathrm{v}$ \\
\hline 16069 & 1467 & Ia & Ia-norm & $11.5 \pm 0.2$ & $12 \pm 26$ & $0.1288 \pm 0.0001$ & $0.124 \pm 0.004$ & $\mathrm{~s}$ \\
\hline 16069 & 1651 & Ia & Ia & $28.3 \pm 0.2$ & - & $0.1288 \pm 0.0001$ & - & $\mathrm{v}$ \\
\hline 16087 & 1455 & II & IIP & - & $14 \pm 23$ & $0.0554 \pm 0.0001$ & $0.052 \pm 0.005$ & \\
\hline 16163 & 1678 & photo-Ia & Gal & $30.6 \pm 0.3$ & - & $0.1549 \pm 0.0005$ & $0.1557 \pm 0.0006$ & $\mathrm{zg}$ \\
\hline 16165 & 1326 & Ia & Ia-norm & $2.6 \pm 0.1$ & $0 \pm 5$ & $0.157 \pm 0.005$ & $0.161 \pm 0.005$ & $\mathrm{zS}$ \\
\hline 16179 & 1323 & Ic & - & - & - & $0.1370 \pm 0.0005$ & - & $\mathrm{zg}$ \\
\hline 16179 & 1469 & Ic & Ic & - & - & $0.1370 \pm 0.0005$ & - & $\mathrm{v}, \mathrm{zg}$ \\
\hline 16179 & 1570 & Ic & Ic & - & $-1 \pm 3$ & $0.1370 \pm 0.0005$ & $0.13 \pm 0.01$ & $\mathrm{zg}$ \\
\hline 16192 & 1322 & II & - & - & - & $0.0787 \pm 0.0001$ & - & \\
\hline 16192 & 1496 & II & IIP & - & $17 \pm 32$ & $0.0787 \pm 0.0001$ & $0.074 \pm 0.006$ & \\
\hline 16204 & 1500 & photo-non-Ia & Gal & - & - & $0.0546 \pm 0.0002$ & $0.0537 \pm 0.0006$ & $\mathrm{f}$ \\
\hline 16206 & 1501 & Ia & Ia-norm & $6.1 \pm 0.2$ & $11 \pm 9$ & $0.1600 \pm 0.0005$ & $0.165 \pm 0.007$ & $\mathrm{~s}, \mathrm{zg}$ \\
\hline 16215 & 1456 & Ia & Ia-norm & $4.29 \pm 0.09$ & $0 \pm 6$ & $0.0466 \pm 0.0002$ & $0.045 \pm 0.005$ & $\mathrm{~s}$ \\
\hline 16215 & 1630 & Ia & Ia-norm & $24.28 \pm 0.09$ & $31 \pm 8$ & $0.0466 \pm 0.0002$ & $0.046 \pm 0.002$ & $\mathrm{~s}$ \\
\hline 16241 & 1470 & photo-non-Ia & - & - & - & $0.0979 \pm 0.0005$ & - & $\mathrm{zg}$ \\
\hline 16280 & 1471 & Ia & Ia-norm & $5.61 \pm 0.07$ & $8 \pm 11$ & $0.0381 \pm 0.0002$ & $0.036 \pm 0.009$ & $\mathrm{~s}$ \\
\hline 16280 & 1564 & Ia & Ia & $23.89 \pm 0.07$ & $16 \pm 8$ & $0.0381 \pm 0.0002$ & $0.037 \pm 0.008$ & $\mathrm{~s}$ \\
\hline 16287 & 1449 & Ia & Ia-norm & $2.4 \pm 0.1$ & $0 \pm 5$ & $0.1064 \pm 0.0005$ & $0.106 \pm 0.005$ & $\mathrm{zg}$ \\
\hline 16287 & 1569 & Ia & Ia-norm & $3.3 \pm 0.1$ & $0 \pm 5$ & $0.1064 \pm 0.0005$ & $0.109 \pm 0.005$ & $\mathrm{zg}$ \\
\hline 16287 & 1650 & Ia & Ia-norm & $19.5 \pm 0.1$ & $31 \pm 8$ & $0.1064 \pm 0.0005$ & $0.105 \pm 0.003$ & $\mathrm{~s}, \mathrm{zg}$ \\
\hline 16302 & 1473 & photo-Ia & - & - & - & - & - & $\mathrm{v}$ \\
\hline 16314 & 1335 & Ia & Ia & $-10.5 \pm 0.2^{p}$ & $-10 \pm 6$ & $0.0626 \pm 0.0001$ & $0.072 \pm 0.006$ & $\mathrm{f}$ \\
\hline 16314 & 1475 & Ia & Ia & $-4.9 \pm 0.2^{p}$ & $-2 \pm 5$ & $0.0626 \pm 0.0001$ & $0.063 \pm 0.006$ & \\
\hline 16333 & 1367 & Ia & Ia-91T & $-7.4 \pm 0.4$ & $-7 \pm 3$ & $0.0719 \pm 0.0001$ & $0.067 \pm 0.002$ & $\mathrm{~s}$ \\
\hline 16352 & 1478 & Ia & Ia-norm & $4.1 \pm 0.4$ & $1 \pm 4$ & $0.248 \pm 0.005$ & $0.255 \pm 0.007$ & zs \\
\hline 16391 & 1452 & II & - & - & - & $0.12 \pm 0.01$ & - & $\mathrm{zt}$ \\
\hline 16391 & 1565 & II & II & - & $0 \pm 38$ & $0.12 \pm 0.01$ & $0.109 \pm 0.007$ & $\mathrm{zt}$ \\
\hline 16392 & 1365 & Ia & Ia-norm & $-8.4 \pm 0.5^{p}$ & $-1 \pm 5$ & $0.0592 \pm 0.0002$ & $0.057 \pm 0.005$ & $\mathrm{~s}$ \\
\hline 16392 & 1448 & Ia & Ia-norm & $-1.8 \pm 0.5^{p}$ & $-1 \pm 5$ & $0.0592 \pm 0.0002$ & $0.061 \pm 0.005$ & \\
\hline 16392 & 1566 & Ia & NotSN & $16.2 \pm 0.5^{p}$ & - & $0.0592 \pm 0.0002$ & $0.0583 \pm 0.0009$ & $\mathrm{~s}$ \\
\hline 16392 & 1682 & Ia & Ia & $18.0 \pm 0.5^{p}$ & $24 \pm 12$ & $0.0592 \pm 0.0002$ & $0.053 \pm 0.007$ & $\mathrm{~s}$ \\
\hline 16402 & 1505 & Ia & - & $3.5 \pm 0.3$ & - & $0.2645 \pm 0.0005$ & - & $\mathrm{s}, \mathrm{zg}$ \\
\hline 16473 & 1520 & Ia & Ia-norm & $1.3 \pm 0.9$ & $0 \pm 5$ & $0.211 \pm 0.005$ & $0.215 \pm 0.006$ & $\mathrm{~s}, \mathrm{zs}$ \\
\hline 16541 & 1485 & Ia & Ia & $-5 \pm 1^{p}$ & $1 \pm 3$ & $0.128 \pm 0.005$ & $0.131 \pm 0.004$ & zs \\
\hline 16578 & 1516 & Ia & Ia-norm & $2.9 \pm 0.9^{p}$ & $0 \pm 5$ & $0.1747 \pm 0.0005$ & $0.178 \pm 0.006$ & $\mathrm{~s}, \mathrm{zg}$ \\
\hline 16619 & 1519 & Ia & Ia-norm & $-3.6 \pm 0.5^{p}$ & $0 \pm 4$ & $0.101 \pm 0.005$ & $0.106 \pm 0.004$ & $\mathrm{zs}$ \\
\hline 16619 & 1528 & Ia & Ia-norm & $-6.6 \pm 0.5^{p}$ & $0 \pm 4$ & $0.101 \pm 0.005$ & $0.104 \pm 0.005$ & zs \\
\hline 16637 & 1514 & Ia & Ia-norm & $-1 \pm 1$ & $-6 \pm 9$ & $0.2430 \pm 0.0005$ & $0.24 \pm 0.01$ & $z g, f$ \\
\hline 16641 & 1518 & Ia & Ia & $-3.2 \pm 0.5^{p}$ & - & $0.1265 \pm 0.0005$ & - & $\mathrm{v}, \mathrm{zg}$ \\
\hline 16641 & 1530 & Ia & Ia-norm & $-6.1 \pm 0.5^{p}$ & $-1 \pm 5$ & $0.1265 \pm 0.0005$ & $0.118 \pm 0.005$ & $\mathrm{~s}, \mathrm{zg}$ \\
\hline 16641 & 1649 & Ia & Ia-norm & $11.9 \pm 0.5^{p}$ & $14 \pm 39$ & $0.1265 \pm 0.0005$ & $0.123 \pm 0.005$ & $\mathrm{~s}, \mathrm{zg}$ \\
\hline 16668 & 1561 & II & IIn & - & $318 \pm 100$ & $0.1518 \pm 0.0005$ & $0.169 \pm 0.005$ & $\mathrm{zg}$ \\
\hline 16692 & 1489 & Ia & Ia-norm & $-6.5 \pm 0.3^{p}$ & $-1 \pm 4$ & $0.0341 \pm 0.0002$ & $0.033 \pm 0.003$ & \\
\hline 16737 & 1599 & Ia & Ia-norm & $-5 \pm 1^{p}$ & $-2 \pm 5$ & $0.200 \pm 0.005$ & $0.191 \pm 0.007$ & zs \\
\hline 16741 & 1523 & - & - & - & - & - & - & \\
\hline 16748 & 1574 & Ia & Gal & $0 \pm 2^{p}$ & - & $0.2320 \pm 0.0005$ & $0.232 \pm 0.004$ & $z g, f$ \\
\hline 16774 & 1606 & Ia & - & $-9 \pm 2^{p}$ & - & $0.2146 \pm 0.0005$ & - & $\mathrm{zg}, \mathrm{f}$ \\
\hline 16778 & 1542 & - & - & - & - & $0.0861 \pm 0.0005$ & - & $\mathrm{zg}$ \\
\hline 16778 & 1568 & - & - & - & - & $0.0861 \pm 0.0005$ & - & $\mathrm{zg}$ \\
\hline 16793 & 1603 & Ia & Ia-norm & $-2.5 \pm 0.7^{p}$ & $-1 \pm 6$ & $0.222 \pm 0.005$ & $0.221 \pm 0.009$ & zS \\
\hline 16838 & 1522 & II & IIn (sn98S) & - & $-12.8 \pm 0.4$ & $0.15 \pm 0.01$ & $0.14945 \pm 0.00007$ & $\mathrm{zt}, \mathrm{f}$ \\
\hline
\end{tabular}


Table A.2. continued.

\begin{tabular}{|c|c|c|c|c|c|c|c|c|}
\hline ID & SPID & SDSS type $^{a}$ & $\begin{array}{l}\text { NTT/NOT } \\
\text { type }^{b}\end{array}$ & $\mathrm{LC}_{\text {epoch }}{ }^{c}$ & $\begin{array}{l}\text { SNID } \\
\text { epoch }^{d}\end{array}$ & $\operatorname{SDSS} z^{e}$ & SNID $z$ & Notes $^{f}$ \\
\hline 16857 & 1538 & - & - & - & - & $0.0753 \pm 0.0002$ & - & \\
\hline 16867 & 1541 & - & - & - & - & $0.1195 \pm 0.0005$ & - & $\mathrm{zg}$ \\
\hline 16872 & 1539 & Ia & Ia-norm & $-4.6 \pm 1.0^{p}$ & $-1 \pm 4$ & $0.1266 \pm 0.0005$ & $0.119 \pm 0.004$ & $\mathrm{zg}$ \\
\hline 16956 & 1562 & Ia & Ia-norm & $-0.2 \pm 0.7^{p}$ & $1 \pm 6$ & $0.1087 \pm 0.0005$ & $0.107 \pm 0.006$ & $\mathrm{zg}$ \\
\hline 16979 & 1597 & photo-non-Ia & - & - & - & $0.4874 \pm 0.0005$ & - & $\mathrm{zg}$ \\
\hline 16988 & 1595 & $\mathrm{Ib}$ & - & - & - & $0.05836 \pm 0.00008$ & - & \\
\hline 16988 & 1652 & $\mathrm{Ib}$ & Ib-norm & - & $12 \pm 13$ & $0.05836 \pm 0.00008$ & $0.058 \pm 0.007$ & \\
\hline 17117 & 1679 & Ia & Ia-norm & $7.4 \pm 0.5^{p}$ & $-1 \pm 5$ & $0.14017 \pm 0.00004$ & $0.143 \pm 0.005$ & \\
\hline 17135 & 1648 & Ia & Ia-norm & - & $-1 \pm 5$ & $0.03092 \pm 0.00008$ & $0.032 \pm 0.004$ & \\
\hline 17167 & 2250 & II & IIP & - & $68 \pm 44$ & $0.0849 \pm 0.0005$ & $0.076 \pm 0.003$ & $\mathrm{zg}$ \\
\hline 17170 & 1879 & - & - & - & - & $0.2205 \pm 0.0005$ & - & $\mathrm{zg}$ \\
\hline 17176 & 1812 & Ia & Ia & $16 \pm 2^{p}$ & $26 \pm 9$ & $0.0935 \pm 0.0001$ & $0.105 \pm 0.007$ & $\mathrm{~s}$ \\
\hline 17200 & 1796 & II & IIP & - & $48 \pm 50$ & $0.08469 \pm 0.00009$ & $0.084 \pm 0.003$ & \\
\hline 17206 & 1788 & photo-Ia & - & $9.0 \pm 0.4$ & - & $0.1564 \pm 0.0001$ & - & \\
\hline 17218 & 1794 & Ia & Ia & $10.5 \pm 0.5$ & - & $0.1783 \pm 0.0005$ & - & $\mathrm{v}, \mathrm{zg}$ \\
\hline 17220 & 1791 & Ia & Ia-norm & $4.2 \pm 0.2$ & $2 \pm 6$ & $0.1783 \pm 0.0005$ & $0.179 \pm 0.007$ & $\mathrm{~s}, \mathrm{zg}$ \\
\hline 17223 & 1793 & Ia & Ia-norm & $13 \pm 1^{p}$ & $11 \pm 51$ & $0.235 \pm 0.005$ & $0.236 \pm 0.007$ & zs \\
\hline 17237 & 1830 & photo-non-Ia & NotSN & - & - & $0.2516 \pm 0.0005$ & $0.253 \pm 0.001$ & $\mathrm{zg}$ \\
\hline 17245 & 2234 & photo-non-Ia & Gal & - & - & $0.2784 \pm 0.0005$ & $0.2784 \pm 0.0004$ & $\mathrm{zg}$ \\
\hline 17247 & 1799 & photo-Ia & Gal & $23 \pm 2^{p}$ & - & $0.2021 \pm 0.0005$ & $0.2018 \pm 0.0007$ & $\mathrm{zg}$ \\
\hline 17253 & 1898 & Ia & Ia-norm & $18.1 \pm 0.9^{p}$ & $21 \pm 27$ & $0.1560 \pm 0.0005$ & $0.158 \pm 0.005$ & $\mathrm{~s}, \mathrm{zg}$ \\
\hline 17254 & 1813 & Ia? & Ia? & $6 \pm 4^{p}$ & - & $0.2691 \pm 0.0005$ & - & $\mathrm{v}, \mathrm{zg}$ \\
\hline 17332 & 1899 & Ia & Ia-norm & $3.3 \pm 0.2$ & $2 \pm 6$ & $0.1828 \pm 0.0001$ & $0.183 \pm 0.007$ & \\
\hline 17351 & 1769 & - & - & - & - & $0.1832 \pm 0.0007$ & - & $\mathrm{zg}$ \\
\hline 17366 & 1782 & Ia & Ia-norm & $8.9 \pm 0.2$ & $6 \pm 53$ & $0.1393 \pm 0.0002$ & $0.143 \pm 0.008$ & \\
\hline 17389 & 1811 & Ia & Ia & $7.0 \pm 0.3$ & $6 \pm 5$ & $0.1706 \pm 0.0005$ & $0.171 \pm 0.007$ & $\mathrm{~s}, \mathrm{zg}$ \\
\hline 17391 & 1872 & Ia & Ia-norm & $16 \pm 2^{p}$ & $20 \pm 26$ & $0.1849 \pm 0.0005$ & $0.193 \pm 0.005$ & $\mathrm{zg}$ \\
\hline 17422 & 1785 & II & II & - & - & $0.1493 \pm 0.0005$ & - & $\mathrm{v}, \mathrm{zg}$ \\
\hline 17435 & 1902 & Ia & Ia-norm & $2.7 \pm 0.2$ & $1 \pm 4$ & $0.2180 \pm 0.0005$ & $0.224 \pm 0.006$ & $\mathrm{zg}$ \\
\hline 17436 & 1790 & - & - & - & - & $0.1449 \pm 0.0006$ & - & $\mathrm{v}, \mathrm{zg}$ \\
\hline 17464 & 1853 & Ia & Ia & $3 \pm 1^{p}$ & $7 \pm 5$ & $0.2549 \pm 0.0005$ & $0.250 \pm 0.003$ & $\mathrm{zg}$ \\
\hline 17486 & 1854 & photo-non-Ia & - & - & - & $0.4476 \pm 0.0002$ & - & $\mathrm{f}$ \\
\hline 17497 & 1837 & Ia & Ia-norm & $-2.38 \pm 0.09$ & $-1 \pm 5$ & $0.1448 \pm 0.0001$ & $0.146 \pm 0.005$ & $\mathrm{~s}$ \\
\hline 17500 & 2249 & Ia & Ia-norm & $67.9 \pm 0.1^{p}$ & $66 \pm 35$ & $0.0441 \pm 0.0002$ & $0.043 \pm 0.005$ & \\
\hline 17535 & 1838 & photo-II & - & - & - & - & - & \\
\hline 17548 & 1825 & Ic & Gal & - & - & $0.0393 \pm 0.0005$ & $0.0392 \pm 0.0003$ & $\mathrm{zg}$ \\
\hline 17548 & 2231 & Ic & Ic & - & - & $0.0393 \pm 0.0005$ & - & $\mathrm{v}, \mathrm{zg}$ \\
\hline 17548 & 2293 & Ic & Ic & - & - & $0.0393 \pm 0.0005$ & - & $\mathrm{v}, \mathrm{zg}$ \\
\hline 17552 & 1789 & Ia & Ia-norm & $3.5 \pm 0.5$ & $0 \pm 5$ & $0.2542 \pm 0.0005$ & $0.255 \pm 0.007$ & $\mathrm{~s}, \mathrm{zg}$ \\
\hline 17568 & 1810 & Ia & Ia-norm & $1 \pm 4$ & $-1 \pm 4$ & $0.1445 \pm 0.0005$ & $0.141 \pm 0.004$ & $\mathrm{~s}, \mathrm{zg}$ \\
\hline 17605 & 1809 & Ia & Ia & $8 \pm 2$ & - & $0.1465 \pm 0.0005$ & - & $\mathrm{v}, \mathrm{zg}$ \\
\hline 17627 & 1841 & II & IIP & - & $5 \pm 60$ & $0.06966 \pm 0.00008$ & $0.070 \pm 0.003$ & \\
\hline 17629 & 1851 & Ia & Ia-norm & $-7.13 \pm 0.07$ & $1 \pm 6$ & $0.13690 \pm 0.00007$ & $0.136 \pm 0.006$ & $\mathrm{~s}$ \\
\hline 17647 & 1875 & photo-Ia & - & - & - & - & - & $\mathrm{v}$ \\
\hline 17703 & 1881 & photo-II & - & - & - & - & - & \\
\hline 17745 & 2161 & Ia & Ia & $15.11 \pm 0.08$ & $16 \pm 32$ & $0.0636 \pm 0.0005$ & $0.063 \pm 0.005$ & $\mathrm{~s}, \mathrm{zg}$ \\
\hline 17746 & 1873 & - & - & - & - & $0.157 \pm 0.005$ & - & zs \\
\hline 17784 & 1842 & Ia & Ia-norm & $-5.55 \pm 0.03$ & $-1 \pm 5$ & $0.03710 \pm 0.00007$ & $0.029 \pm 0.005$ & \\
\hline 17790 & 1887 & Ia & Ia-norm & $1.0 \pm 0.4$ & $0 \pm 5$ & $0.178 \pm 0.005$ & $0.177 \pm 0.006$ & zs \\
\hline 17794 & 1906 & photo-Ibc & - & - & - & - & - & \\
\hline 17811 & 1816 & Ia & Ia & $4.6 \pm 0.8$ & $1 \pm 5$ & $0.2132 \pm 0.0005$ & $0.205 \pm 0.006$ & $\mathrm{zg}$ \\
\hline 17814 & 1901 & photo-non-Ia & Gal & - & - & $0.1069 \pm 0.0005$ & $0.1071 \pm 0.0005$ & $\mathrm{zg}$ \\
\hline 17825 & 1819 & Ia & Ia-norm & $-4.9 \pm 0.1$ & $-1 \pm 5$ & $0.161 \pm 0.005$ & $0.162 \pm 0.005$ & zs \\
\hline 17854 & 2230 & - & - & - & - & - & - & $\mathrm{v}$ \\
\hline 17875 & 1817 & Ia & Ia-norm & $0.3 \pm 0.3$ & $0 \pm 5$ & $0.2323 \pm 0.0005$ & $0.223 \pm 0.008$ & $\mathrm{~s}, \mathrm{zg}$ \\
\hline 17880 & 1843 & Ia & Ia-norm & $-1.87 \pm 0.06$ & $0 \pm 6$ & $0.07265 \pm 0.00006$ & $0.061 \pm 0.005$ & \\
\hline 17880 & 1957 & Ia & Ia-norm & $1.16 \pm 0.06$ & $-1 \pm 6$ & $0.07265 \pm 0.00006$ & $0.065 \pm 0.005$ & $\mathrm{~s}$ \\
\hline 17880 & 2194 & Ia & Ia & $24.44 \pm 0.06$ & $30 \pm 7$ & $0.07265 \pm 0.00006$ & $0.072 \pm 0.005$ & $\mathrm{~s}$ \\
\hline 17886 & 1844 & Ia & Ia-norm & $-4.48 \pm 0.04$ & $-1 \pm 5$ & $0.0408 \pm 0.0002$ & $0.040 \pm 0.005$ & \\
\hline 17924 & 1826 & photo-non-Ia & Gal & - & - & $0.1456 \pm 0.0005$ & $0.1444 \pm 0.0008$ & $z g, f$ \\
\hline 17973 & 1926 & photo-non-Ia & NotSN & - & - & $0.1456 \pm 0.0005$ & $0.149 \pm 0.001$ & $\mathrm{zg}$ \\
\hline 17973 & 1942 & photo-non-Ia & Gal & - & - & $0.1456 \pm 0.0005$ & $0.148 \pm 0.001$ & $\mathrm{zg}, \mathrm{f}$ \\
\hline 18109 & 1940 & II & - & - & - & $0.0680 \pm 0.0002$ & - & \\
\hline 18325 & 2277 & Ia & Ia-norm & $8.6 \pm 0.3$ & $10 \pm 51$ & $0.255 \pm 0.005$ & $0.259 \pm 0.008$ & $\mathrm{zS}$ \\
\hline 18457 & 2285 & II & - & - & - & $0.08097 \pm 0.00008$ & - & \\
\hline 18466 & 2270 & Ia & Ia-norm & $4.5 \pm 0.3$ & $0 \pm 5$ & $0.213 \pm 0.005$ & $0.218 \pm 0.006$ & zs \\
\hline
\end{tabular}


Table A.2. continued.

\begin{tabular}{|c|c|c|c|c|c|c|c|c|}
\hline ID & SPID & SDSS type $^{a}$ & $\begin{array}{l}\text { NTT/NOT } \\
\text { type }^{b}\end{array}$ & $\mathrm{LC}_{\text {epoch }}{ }^{c}$ & $\begin{array}{l}\text { SNID } \\
\text { epoch }^{d}\end{array}$ & $\operatorname{SDSS} z^{e}$ & SNID $z$ & Notes $^{f}$ \\
\hline 18590 & 2248 & II & - & - & - & $0.0572 \pm 0.0002$ & - & \\
\hline 18596 & 2227 & II & IIP & - & $7 \pm 21$ & $0.027 \pm 0.005$ & $0.023 \pm 0.004$ & $\mathrm{zs}$ \\
\hline 18647 & 2271 & photo-non-Ia & - & - & - & $0.2128 \pm 0.0002$ & - & \\
\hline 18697 & 2171 & Ia & Ia & $4.27 \pm 0.08$ & $8 \pm 3$ & $0.10725 \pm 0.00005$ & $0.106 \pm 0.005$ & $\mathrm{~s}$ \\
\hline 18768 & 2135 & Ia & Ia-norm & $6.7 \pm 0.3$ & $6 \pm 5$ & $0.198 \pm 0.005$ & $0.199 \pm 0.006$ & zs \\
\hline 18787 & 2150 & Ia & Ia-norm & $0.2 \pm 0.4$ & $-1 \pm 4$ & $0.2073 \pm 0.0005$ & $0.196 \pm 0.006$ & $\mathrm{zg}$ \\
\hline 18804 & 2148 & Ia & Ia-norm & $-4.99 \pm 0.09$ & $-1 \pm 6$ & $0.2052 \pm 0.0005$ & $0.194 \pm 0.007$ & $\mathrm{zg}$ \\
\hline 18903 & 2247 & $\mathrm{Ia} ?$ & Ia? & $-3.9 \pm 0.3$ & - & $0.1564 \pm 0.0002$ & - & $\mathrm{v}$ \\
\hline 18965 & 2279 & Ia & Ia-norm & $-4.3 \pm 0.2$ & $-1 \pm 4$ & $0.2066 \pm 0.0005$ & $0.207 \pm 0.005$ & $\mathrm{~s}, \mathrm{zg}$ \\
\hline 19003 & 2235 & Ia & Ia-norm & $-6.4 \pm 0.1$ & $-5 \pm 4$ & $0.0612 \pm 0.0002$ & $0.056 \pm 0.005$ & $\mathrm{~s}$ \\
\hline 19003 & 2290 & Ia & Ia-norm & $-4.6 \pm 0.1$ & $-6 \pm 5$ & $0.0612 \pm 0.0002$ & $0.060 \pm 0.004$ & $\mathrm{~s}$ \\
\hline 19008 & 2284 & Ia & Ia-norm & $-2.1 \pm 0.3$ & $-6 \pm 8$ & $0.2322 \pm 0.0005$ & $0.230 \pm 0.009$ & $\mathrm{zg}$ \\
\hline 19023 & 2236 & Ia & Ia-norm & $-1.7 \pm 0.2$ & $-2.9 \pm 0.5$ & $0.243 \pm 0.005$ & $0.264 \pm 0.007$ & $z s, f$ \\
\hline 19051 & 2297 & - & - & - & - & $0.2773 \pm 0.0005$ & - & $\mathrm{zg}, \mathrm{f}$ \\
\hline 19101 & 2268 & Ia & Ia-norm & $-6.0 \pm 0.1$ & $0 \pm 5$ & $0.187 \pm 0.005$ & $0.189 \pm 0.006$ & zs \\
\hline 19149 & 2275 & Ia & Ia-91T & $-7.1 \pm 0.1$ & $-6 \pm 2$ & $0.196 \pm 0.005$ & $0.204 \pm 0.001$ & zs \\
\hline 19155 & 2252 & Ia & Ia-norm & $-11.66 \pm 0.04$ & $-5 \pm 4$ & $0.0769 \pm 0.0002$ & $0.070 \pm 0.001$ & \\
\hline 19155 & 2607 & Ia & Ia & $18.69 \pm 0.04$ & $16 \pm 29$ & $0.0769 \pm 0.0002$ & $0.077 \pm 0.004$ & $\mathrm{~s}$ \\
\hline 19155 & 2720 & Ia & Ia-norm & $38.37 \pm 0.04$ & $34 \pm 12$ & $0.0769 \pm 0.0002$ & $0.079 \pm 0.002$ & $\mathrm{~s}$ \\
\hline 19221 & 2274 & - & - & - & - & $0.0433 \pm 0.0005$ & - & $\mathrm{v}, \mathrm{zg}$ \\
\hline 19222 & 2299 & II & IIP & - & $0 \pm 2$ & $0.1683 \pm 0.0005$ & $0.162 \pm 0.004$ & $\mathrm{zg}$ \\
\hline 19230 & 2282 & Ia & Ia-norm & $-1.9 \pm 0.2$ & $1 \pm 5$ & $0.2215 \pm 0.0005$ & $0.223 \pm 0.006$ & $\mathrm{~s}, \mathrm{zg}$ \\
\hline 19282 & 2280 & Ia & Ia-norm & $-8.16 \pm 0.10$ & $-2 \pm 5$ & $0.1864 \pm 0.0002$ & $0.177 \pm 0.006$ & \\
\hline 19323 & 2296 & $\mathrm{Ib}$ & Ib-norm & - & $11 \pm 14$ & $0.08679 \pm 0.00009$ & $0.073 \pm 0.007$ & \\
\hline 19341 & 2298 & Ia & Ia-norm & $-2.4 \pm 0.3$ & $-1 \pm 5$ & $0.228 \pm 0.005$ & $0.229 \pm 0.005$ & $\mathrm{~s}, \mathrm{zs}$ \\
\hline 19353 & 2281 & Ia & Ia-norm & $-7 \pm 2$ & $-2 \pm 5$ & $0.1540 \pm 0.0001$ & $0.149 \pm 0.005$ & $\mathrm{~s}$ \\
\hline 19381 & 2283 & Ia & Ia-norm & $-3.49 \pm 0.07$ & $0 \pm 4$ & $0.210 \pm 0.005$ & $0.212 \pm 0.005$ & $\mathrm{zs}$ \\
\hline 19899 & 2550 & Ia & Ia-norm & $1.22 \pm 0.09$ & $0 \pm 4$ & $0.089 \pm 0.005$ & $0.092 \pm 0.003$ & zs \\
\hline 19913 & 2585 & Ia & Ia-norm & $9.6 \pm 0.3$ & $9 \pm 15$ & $0.2038 \pm 0.0005$ & $0.208 \pm 0.006$ & $\mathrm{zg}$ \\
\hline 19953 & 2602 & Ia & Ia-norm & $4.2 \pm 0.1$ & $0 \pm 5$ & $0.120 \pm 0.005$ & $0.124 \pm 0.005$ & zs \\
\hline 19968 & 2549 & Ia & Ia-norm & $5.24 \pm 0.05$ & $1 \pm 6$ & $0.0560 \pm 0.0001$ & $0.059 \pm 0.005$ & \\
\hline 20039 & 2584 & Ia & Ia-norm & $7.6 \pm 0.3$ & $6 \pm 5$ & $0.2477 \pm 0.0005$ & $0.250 \pm 0.007$ & $\mathrm{~s}, \mathrm{zg}$ \\
\hline 20040 & 2612 & Ia & Ia & $6.4 \pm 0.5$ & $-2 \pm 5$ & $0.2880 \pm 0.0005$ & $0.288 \pm 0.006$ & $\mathrm{~s}, \mathrm{zg}$ \\
\hline 20052 & 2537 & photo-non-Ia & NotSN & - & - & $0.1574 \pm 0.0002$ & $0.1578 \pm 0.0009$ & \\
\hline 20052 & 2538 & photo-non-Ia & - & - & - & $0.1574 \pm 0.0002$ & - & \\
\hline 20088 & 2546 & Ia & - & $8.4 \pm 0.2$ & - & $0.2444 \pm 0.0005$ & - & $\mathrm{zg}$ \\
\hline 20097 & 2587 & - & - & - & - & $0.221 \pm 0.005$ & - & zs \\
\hline 20142 & 2586 & Ia & $\begin{array}{l}\text { Ia-norm } \\
(\text { sn90N) }\end{array}$ & $4.7 \pm 0.4$ & -13.2 & $0.3139 \pm 0.0005$ & $0.32 \pm 0.01$ & $\mathrm{~s}, \mathrm{zg}, \mathrm{f}$ \\
\hline 20144 & 2541 & Ia & Ia-norm & $1.1 \pm 0.3$ & $-1 \pm 4$ & $0.220 \pm 0.005$ & $0.225 \pm 0.005$ & $\mathrm{~s}, \mathrm{zs}$ \\
\hline 20227 & 2568 & Ia & Ia-norm & $7.5 \pm 0.4$ & $7 \pm 4$ & $0.2764 \pm 0.0005$ & $0.282 \pm 0.006$ & $\mathrm{~s}, \mathrm{zg}$ \\
\hline 20345 & 2567 & Ia & Ia-norm & $-0.7 \pm 0.3$ & $0 \pm 5$ & $0.265 \pm 0.005$ & $0.267 \pm 0.008$ & zs \\
\hline 20364 & 2581 & Ia & Ia-norm & $-1.3 \pm 0.6$ & $0 \pm 5$ & $0.2181 \pm 0.0009$ & $0.218 \pm 0.007$ & $\mathrm{zg}$ \\
\hline 20376 & 2582 & Ia & Ia-norm & $3.8 \pm 0.5$ & $2 \pm 6$ & $0.2109 \pm 0.0005$ & $0.204 \pm 0.007$ & $\mathrm{~s}, \mathrm{zg}$ \\
\hline 20388 & 2611 & photo-non-Ia & - & - & - & $0.1787 \pm 0.0005$ & - & $\mathrm{zg}$ \\
\hline 20430 & 2543 & Ia & Ia-norm & $1.4 \pm 0.3$ & $0 \pm 5$ & $0.164 \pm 0.005$ & $0.168 \pm 0.005$ & zs \\
\hline 20474 & 2563 & - & - & - & - & $0.2713 \pm 0.0005$ & - & $z g, f$ \\
\hline 20474 & 2714 & - & - & - & - & $0.2713 \pm 0.0005$ & - & $\mathrm{zg}$ \\
\hline 20474 & 3003 & - & - & - & - & $0.2713 \pm 0.0005$ & - & $\mathrm{zg}$ \\
\hline 20530 & 2547 & II & II & - & - & $0.06135 \pm 0.00008$ & - & $\mathrm{v}$ \\
\hline 20530 & 2571 & II & II & - & - & $0.06135 \pm 0.00008$ & - & $\mathrm{v}$ \\
\hline 20575 & 2540 & Ia & Ia-norm & $2.3 \pm 0.3$ & $0 \pm 4$ & $0.1988 \pm 0.0005$ & $0.204 \pm 0.005$ & $\mathrm{~s}, \mathrm{zg}$ \\
\hline 20575 & 3005 & Ia & - & $0.7 \pm 0.3$ & - & $0.1988 \pm 0.0005$ & - & $\mathrm{s}, \mathrm{zg}$ \\
\hline 20625 & 2551 & Ia & Ia-norm & $-5.4 \pm 0.1$ & $-1 \pm 4$ & $0.1082 \pm 0.0002$ & $0.108 \pm 0.004$ & $\mathrm{~s}$ \\
\hline 20625 & 2604 & $\mathrm{Ia}$ & Ia-norm & $-3.6 \pm 0.1$ & $-1 \pm 4$ & $0.1082 \pm 0.0002$ & $0.110 \pm 0.004$ & $\mathrm{~s}$ \\
\hline 20677 & 2536 & Ic & $\mathrm{Ib} / \mathrm{c}$ & - & - & $0.0804 \pm 0.0001$ & - & $\mathrm{v}$ \\
\hline 20678 & 2610 & photo-Ia & - & $3.9 \pm 0.3$ & - & $0.2056 \pm 0.0001$ & - & \\
\hline 20687 & 2596 & Ia & Ia-norm & $-0.2 \pm 0.5$ & $-1 \pm 4$ & $0.1918 \pm 0.0005$ & $0.194 \pm 0.005$ & $\mathrm{~s}, \mathrm{zg}$ \\
\hline 20687 & 2597 & Ia & Ia-norm & $-1.3 \pm 0.5$ & $2 \pm 4$ & $0.1918 \pm 0.0005$ & $0.191 \pm 0.002$ & $\mathrm{~s}, \mathrm{zg}$ \\
\hline 20718 & 2577 & - & Gal & - & - & $0.0888 \pm 0.0001$ & $0.0892 \pm 0.0003$ & f \\
\hline 20718 & 2593 & - & - & - & - & $0.0888 \pm 0.0001$ & - & \\
\hline 20764 & 2594 & Ia & Ia-norm & $-2.5 \pm 0.3$ & $-1 \pm 5$ & $0.1664 \pm 0.0005$ & $0.170 \pm 0.005$ & $\mathrm{~s}, \mathrm{zg}$ \\
\hline 20834 & 2598 & Ia & Ia & $-3 \pm 1^{p}$ & - & $0.1909 \pm 0.0005$ & - & $\mathrm{v}, \mathrm{zg}$ \\
\hline 20862 & 2600 & Ia & Ia-norm & $-4 \pm 1^{p}$ & $-1 \pm 4$ & $0.2665 \pm 0.0005$ & $0.266 \pm 0.005$ & $\mathrm{~s}, \mathrm{zg}$ \\
\hline 20909 & 2580 & photo-non-Ia & - & - & - & $0.1586 \pm 0.0005$ & - & $\mathrm{zg}$ \\
\hline 20978 & 2609 & Ia & Ia-pec & $-2.6 \pm 0.7^{p}$ & $-5 \pm 2$ & $0.324 \pm 0.005$ & $0.314 \pm 0.001$ & $\mathrm{zs}$ \\
\hline
\end{tabular}


Table A.2. continued.

\begin{tabular}{|c|c|c|c|c|c|c|c|c|}
\hline ID & SPID & SDSS type $^{a}$ & $\begin{array}{l}\text { NTT/NOT } \\
\text { type }^{b}\end{array}$ & $\mathrm{LC}_{\mathrm{epoch}}{ }^{c}$ & $\begin{array}{l}\text { SNID } \\
\text { epoch }^{d}\end{array}$ & $\operatorname{SDSS} z^{e}$ & $\operatorname{SNID} z$ & Notes $^{f}$ \\
\hline 21006 & 2566 & Ia & Ia-norm & $1.7 \pm 0.4$ & $0 \pm 4$ & $0.291 \pm 0.005$ & $0.295 \pm 0.005$ & $\mathrm{ZS}$ \\
\hline 21033 & 2565 & Ia & Ia-norm & $-3.3 \pm 0.4$ & $-2 \pm 4$ & $0.229 \pm 0.005$ & $0.231 \pm 0.006$ & zS \\
\hline 21034 & 2719 & Ia & Ia & $13.3 \pm 0.2$ & - & $0.10858 \pm 0.00006$ & - & $\mathrm{v}$ \\
\hline 21034 & 2733 & Ia & Ia & $15.1 \pm 0.2$ & $16 \pm 13$ & $0.10858 \pm 0.00006$ & $0.111 \pm 0.004$ & $\mathrm{~s}$ \\
\hline 21042 & 2564 & Ia & Ia-norm & $-6.4 \pm 1.0$ & $-1 \pm 5$ & $0.3109 \pm 0.0005$ & $0.310 \pm 0.007$ & $\mathrm{zg}$ \\
\hline 21058 & 2579 & photo-non-Ia & - & - & - & $0.1643 \pm 0.0005$ & - & $\mathrm{zg}$ \\
\hline 21058 & 2595 & photo-non-Ia & - & - & - & $0.1643 \pm 0.0005$ & - & $\mathrm{v}, \mathrm{zg}$ \\
\hline 21062 & 2613 & Ia & Ia-norm & $-5.3 \pm 0.2$ & $0 \pm 6$ & $0.1388 \pm 0.0001$ & $0.154 \pm 0.007$ & $\mathrm{~s}$ \\
\hline 21064 & 2532 & II & II & - & - & $0.07930 \pm 0.00008$ & - & $\mathrm{v}$ \\
\hline 21064 & 2533 & II & IIP & - & $1 \pm 3$ & $0.07930 \pm 0.00008$ & $0.077 \pm 0.002$ & \\
\hline 21362 & 2636 & II & - & - & - & $0.0867 \pm 0.0005$ & - & $\mathrm{zg}$ \\
\hline 21362 & 2697 & II & IIP & - & $8 \pm 27$ & $0.0867 \pm 0.0005$ & $0.082 \pm 0.006$ & $\mathrm{zg}$ \\
\hline 21422 & 2599 & Ia & Ia-norm & $-4 \pm 1$ & $-2 \pm 5$ & $0.267 \pm 0.005$ & $0.265 \pm 0.008$ & zS \\
\hline 21502 & 2574 & Ia & Ia-norm & $-8.6 \pm 0.2$ & $-1 \pm 4$ & $0.089 \pm 0.001$ & $0.090 \pm 0.004$ & $\mathrm{~s}$ \\
\hline 21502 & 2575 & Ia & Ia-norm & $-7.7 \pm 0.2$ & $-1 \pm 5$ & $0.089 \pm 0.001$ & $0.091 \pm 0.004$ & $\mathrm{~s}$ \\
\hline 21502 & 2717 & Ia & Ia-norm & $13.7 \pm 0.2$ & $16 \pm 8$ & $0.089 \pm 0.001$ & $0.091 \pm 0.004$ & $\mathrm{~s}$ \\
\hline 21596 & 2588 & photo-non-Ia & - & - & - & $0.0633 \pm 0.0002$ & - & $\mathrm{f}$ \\
\hline 21596 & 2589 & photo-non-Ia & $\mathrm{Gal}$ & - & - & $0.0633 \pm 0.0002$ & $0.0673 \pm 0.0008$ & $\mathrm{f}$ \\
\hline 21669 & 2591 & Ia & Ia-norm & $-8 \pm 2^{p}$ & $-3 \pm 4$ & $0.1242 \pm 0.0002$ & $0.105 \pm 0.006$ & $\mathrm{~s}$ \\
\hline 21669 & 2722 & Ia & Ia-norm & $14 \pm 2^{p}$ & $13 \pm 17$ & $0.1242 \pm 0.0002$ & $0.119 \pm 0.004$ & $\mathrm{~s}$ \\
\hline 21766 & 2638 & - & - & - & - & $0.12788 \pm 0.00006$ & - & \\
\hline 21810 & 2724 & Ia & Ia & $3.0 \pm 0.4^{p}$ & $11 \pm 3$ & $0.175 \pm 0.005$ & $0.180 \pm 0.008$ & ZS \\
\hline 21814 & 2702 & Ia & Ia & $11.5 \pm 0.2$ & - & $0.1021 \pm 0.0005$ & - & $\mathrm{v}, \mathrm{zg}$ \\
\hline 21839 & 2716 & Ia & Ia-norm & $4 \pm 1^{p}$ & $0 \pm 5$ & $0.0935 \pm 0.0005$ & $0.096 \pm 0.005$ & $\mathrm{~s}, \mathrm{zg}$ \\
\hline 21861 & 2723 & Ia & Ia-norm & $1 \pm 2^{p}$ & $-1 \pm 5$ & $0.188 \pm 0.005$ & $0.192 \pm 0.005$ & $\mathrm{~s}, \mathrm{zs}$ \\
\hline 21898 & 2704 & - & - & - & - & $0.0388 \pm 0.0002$ & - & \\
\hline 22182 & 2690 & Ia & Ia & $9 \pm 2^{p}$ & - & $0.076 \pm 0.005$ & - & $\mathrm{v}, \mathrm{zs}$ \\
\hline 22284 & 2735 & Ia & Ia-norm & $1 \pm 3^{p}$ & $-1 \pm 5$ & $0.1375 \pm 0.0006$ & $0.137 \pm 0.006$ & $\mathrm{~s}, \mathrm{zg}$ \\
\hline
\end{tabular}

Notes. ${ }^{(a)}$ The overall SDSS type which is based on the results from the NTT/NOT analysis in combination with spectra from other telescopes. ${ }^{(b)}$ The typing of the individual spectra in this analysis based on the result from SNID in combination with a visual inspection. If a subtype could be determined, this is given in the table, otherwise only the type. Thus, when the type is given as solely Ia, this means that SNID could not determine if it is a normal SN Ia or a non-normal (peculiar, SN 1991 T or SN 1991bg). In the cases where all template spectra which could be matched with the input spectrum with SNID belong to the same SN, the name of the SN is written within parenthesis. When the type is NotSN or Gal, this usually means that the transient was too faint, or the observing conditions were not good enough, so that the contribution from the host galaxy dominates the spectrum. ${ }^{(c)}$ Number of days in rest frame from $B$-band maximum obtained from the lightcurve. An age is given when the object has been classified as a SN Ia, as well as there were enough photometry to build a lightcurve. The sign " $p$ " is added after the lightcurve age when the lightcurve lacked photometry either before or after maximum brightness. ${ }^{(d)}$ Number of days in rest frame from $B$-band maximum obtained from SNID. If no error is given, there was only one template fitting the requirements in SNID. ${ }^{(e)}$ The object redshifts from Zheng et al. (in preparation), which have their origin in SDSS DR7 redshifts in combination with measurements of both NTT/NOT spectra and other spectra. ${ }^{(f)}$ Additional information. When the SNID type was determined from the host-galaxy subtracted spectrum, this is marked by an $s$ in the column. If there were few template spectra matching the input spectrum, at which fewer than 5 spectra were used to determine the type, the redshift and/or the age, this is marked with an $f$. When the type obtained from SNID was changed after the visual inspection this is marked with a $v$. When the redshift is not obtained from SDSS DR7, it is marked with $z g$ or $z s$, depending on if it was determined from galaxy lines or SN features. If the redshift was determined through template fitting, this is marked with $z t$. 
A\&A 526, A28 (2011)

Table A.3. Spectral quality.

\begin{tabular}{|c|c|c|c|}
\hline ID & SPID & Host contamination $^{a}$ & Slit loss ${ }^{b}$ \\
\hline 12778 & 692 & 1.0 & 0.4 \\
\hline 12779 & 693 & 0.4 & 0.4 \\
\hline 12781 & 680 & 0.1 & 0.2 \\
\hline 12782 & 681 & 0.9 & 0.3 \\
\hline 12820 & 711 & 0.6 & 0.2 \\
\hline 12842 & 682 & 0.1 & 0.2 \\
\hline 12843 & 727 & 0.3 & 0.4 \\
\hline 12844 & 684 & 1.0 & 0.4 \\
\hline 12853 & 685 & 0.1 & 0.3 \\
\hline 12855 & 716 & 0.7 & 0.3 \\
\hline 12856 & 695 & 0.5 & 0.5 \\
\hline 12860 & 688 & 0.2 & 0.2 \\
\hline 12874 & 689 & 0.2 & 0.2 \\
\hline 12898 & 712 & 0.5 & 0.1 \\
\hline 12907 & 714 & 0.8 & 0.3 \\
\hline 12927 & 690 & 0.5 & 0.3 \\
\hline 12928 & 686 & 0.3 & 0.3 \\
\hline 12930 & 687 & 0.2 & 0.1 \\
\hline 12947 & 691 & 0.7 & 0.5 \\
\hline 12950 & 700 & 0.3 & 0.1 \\
\hline 12950 & 1055 & 0.8 & 0.7 \\
\hline 12978 & 701 & 0.1 & 0.5 \\
\hline 13005 & 702 & 0.5 & 0.2 \\
\hline 13025 & 761 & 0.2 & 0.8 \\
\hline 13044 & 724 & 0.1 & 0.4 \\
\hline 13044 & 1062 & 0.2 & 0.8 \\
\hline 13045 & 734 & 0.8 & 0.6 \\
\hline 13046 & 726 & 1.0 & 0.8 \\
\hline 13070 & 736 & 0.4 & 0.1 \\
\hline 13072 & 723 & 0.2 & 0.2 \\
\hline 13135 & 739 & 0.1 & 0.4 \\
\hline 13135 & 998 & 0.1 & 0.3 \\
\hline 13174 & 766 & 0.8 & 0.1 \\
\hline 13195 & 764 & 0.3 & 0.7 \\
\hline 13195 & 983 & 0.6 & 0.5 \\
\hline 13195 & 1458 & 0.6 & 0.9 \\
\hline 13355 & 1003 & 0.7 & 0.5 \\
\hline 13376 & 1002 & 0.5 & 0.5 \\
\hline 13376 & 1106 & 0.5 & 0.7 \\
\hline 13577 & 1000 & 0.4 & 0.5 \\
\hline 13796 & 1058 & 0.0 & 0.1 \\
\hline 13894 & 1039 & 0.3 & 0.5 \\
\hline 14157 & 1040 & 0.3 & 0.7 \\
\hline 14279 & 1459 & 0.9 & 0.7 \\
\hline 14318 & 1594 & $0.4^{e}$ & 0.1 \\
\hline 14318 & 1653 & $0.4^{e}$ & 0.1 \\
\hline 14318 & 1713 & $0.6^{e}$ & 0.3 \\
\hline 14437 & 1061 & 0.4 & 0.1 \\
\hline 14450 & 991 & 0.5 & 0.3 \\
\hline 14451 & 989 & 0.6 & 0.5 \\
\hline 14492 & 1001 & 0.5 & 0.2 \\
\hline 14598 & 987 & 1.0 & 0.4 \\
\hline 14599 & 988 & 0.0 & 0.1 \\
\hline 14782 & 990 & 0.7 & 0.2 \\
\hline 14846 & 1014 & 0.3 & 0.2 \\
\hline 14871 & 1008 & 0.4 & 0.2 \\
\hline 14979 & 1009 & 0.2 & 0.1 \\
\hline 14984 & 1027 & 0.5 & 0.2 \\
\hline 15031 & 985 & 0.1 & 0.3 \\
\hline 15129 & 1015 & 0.3 & 0.7 \\
\hline 15132 & 1012 & 0.1 & 0.1 \\
\hline 15136 & 1022 & 0.5 & 0.1 \\
\hline 15153 & 1046 & 0.1 & 0.1 \\
\hline 15161 & 1010 & 0.5 & 0.5 \\
\hline 15171 & 1045 & 0.1 & 0.1 \\
\hline 15203 & 1026 & 0.6 & 0.4 \\
\hline
\end{tabular}


L. Östman et al.: NTT and NOT spectroscopy of SDSS-II supernovae

Table A.3. continued.

\begin{tabular}{|c|c|c|c|}
\hline ID & SPID & Host contamination $^{a}$ & Slit loss ${ }^{b}$ \\
\hline 15207 & 1038 & 0.0 & 0.4 \\
\hline 15210 & 1005 & 0.1 & 0.3 \\
\hline 15210 & 1052 & 0.1 & 0.2 \\
\hline 15222 & 1004 & 0.2 & 0.8 \\
\hline 15234 & 1043 & 0.7 & 0.6 \\
\hline 15259 & 1051 & 0.2 & 0.3 \\
\hline 15287 & 1057 & 0.0 & 0.5 \\
\hline 15320 & 1098 & 0.1 & 0.1 \\
\hline 15339 & 1107 & 0.9 & 0.4 \\
\hline 15354 & 1110 & 0.2 & 0.5 \\
\hline 15475 & 1464 & $1.0^{e}$ & 1.0 \\
\hline 15557 & 1532 & 0.0 & 0.2 \\
\hline 16021 & 1355 & 0.5 & 0.1 \\
\hline 16069 & 1358 & 0.6 & 0.8 \\
\hline 16069 & 1467 & 0.5 & 0.4 \\
\hline 16069 & 1651 & 0.9 & 0.6 \\
\hline 16087 & 1455 & 0.3 & 1.0 \\
\hline 16163 & 1678 & $1.0^{e}$ & 0.1 \\
\hline 16165 & 1326 & 0.1 & 0.3 \\
\hline 16179 & 1323 & 0.4 & 0.6 \\
\hline 16179 & 1469 & 0.2 & 0.9 \\
\hline 16179 & 1570 & 0.2 & 1.0 \\
\hline 16192 & 1322 & 0.6 & 0.5 \\
\hline 16192 & 1496 & $0.7^{e}$ & 0.5 \\
\hline 16204 & 1500 & 0.9 & 0.9 \\
\hline 16206 & 1501 & 0.8 & 0.7 \\
\hline 16215 & 1456 & 0.4 & 0.9 \\
\hline 16215 & 1630 & 0.9 & 0.7 \\
\hline 16241 & 1470 & $0.6^{e}$ & 0.9 \\
\hline 16280 & 1471 & 0.7 & 0.8 \\
\hline 16280 & 1564 & 1.0 & 0.7 \\
\hline 16287 & 1449 & 0.0 & 1.0 \\
\hline 16287 & 1569 & 0.0 & 1.0 \\
\hline 16287 & 1650 & 0.6 & 0.4 \\
\hline 16302 & 1473 & 0.3 & 0.4 \\
\hline 16314 & 1335 & 0.1 & 0.2 \\
\hline 16314 & 1475 & 0.0 & 0.2 \\
\hline 16333 & 1367 & 0.8 & 0.4 \\
\hline 16352 & 1478 & 0.1 & 0.5 \\
\hline 16391 & 1452 & 0.1 & 1.0 \\
\hline 16391 & 1565 & $0.0^{e}$ & 0.6 \\
\hline 16392 & 1365 & 0.3 & 0.4 \\
\hline 16392 & 1448 & 0.1 & 0.9 \\
\hline 16392 & 1566 & $0.5^{e}$ & 0.5 \\
\hline 16392 & 1682 & $0.4^{e}$ & 0.2 \\
\hline 16402 & 1505 & 0.4 & 1.0 \\
\hline 16473 & 1520 & 0.3 & 0.1 \\
\hline 16541 & 1485 & 0.2 & 0.4 \\
\hline 16578 & 1516 & $0.6^{e}$ & 0.8 \\
\hline 16619 & 1519 & 0.0 & 0.6 \\
\hline 16619 & 1528 & 0.1 & 0.2 \\
\hline 16637 & 1514 & 0.5 & 1.0 \\
\hline 16641 & 1518 & 0.2 & 0.9 \\
\hline 16641 & 1530 & 0.4 & 0.3 \\
\hline 16641 & 1649 & $0.4^{e}$ & 0.8 \\
\hline 16668 & 1561 & 0.1 & 0.2 \\
\hline 16692 & 1489 & 0.1 & 0.1 \\
\hline 16737 & 1599 & $0.1^{e}$ & 1.0 \\
\hline 16741 & 1523 & 0.0 & 0.2 \\
\hline 16748 & 1574 & $0.4^{e}$ & 0.2 \\
\hline 16774 & 1606 & 0.1 & 0.7 \\
\hline 16778 & 1542 & 0.4 & 0.0 \\
\hline 16778 & 1568 & 0.1 & 0.1 \\
\hline 16793 & 1603 & 0.0 & 0.9 \\
\hline 16838 & 1522 & 0.0 & 0.4 \\
\hline 16857 & 1538 & 0.7 & 1.0 \\
\hline 16867 & 1541 & 0.2 & 0.6 \\
\hline
\end{tabular}


A\&A 526, A28 (2011)

Table A.3. continued.

\begin{tabular}{|c|c|c|c|}
\hline ID & SPID & Host contamination $^{a}$ & Slit loss ${ }^{b}$ \\
\hline 16872 & 1539 & $0.0^{e}$ & 0.8 \\
\hline 16956 & 1562 & 0.2 & 0.1 \\
\hline 16979 & 1597 & 0.2 & 0.8 \\
\hline 16988 & 1595 & 0.8 & 1.0 \\
\hline 16988 & 1652 & $0.8^{e}$ & 1.0 \\
\hline 17117 & 1679 & $0.0^{e}$ & 0.3 \\
\hline 17135 & 1648 & - & 0.4 \\
\hline 17167 & 2250 & 0.6 & 0.5 \\
\hline 17170 & 1879 & 0.8 & 0.4 \\
\hline 17176 & 1812 & 1.0 & 0.3 \\
\hline 17200 & 1796 & 0.9 & 0.5 \\
\hline 17206 & 1788 & 0.6 & 0.4 \\
\hline 17218 & 1794 & 0.8 & 0.3 \\
\hline 17220 & 1791 & 0.9 & 0.1 \\
\hline 17223 & 1793 & 0.3 & 0.2 \\
\hline 17237 & 1830 & 0.6 & 0.2 \\
\hline 17245 & 2234 & 0.5 & 0.6 \\
\hline 17247 & 1799 & 0.7 & 0.2 \\
\hline 17253 & 1898 & 0.7 & 0.4 \\
\hline 17254 & 1813 & 0.9 & 0.3 \\
\hline 17332 & 1899 & 0.0 & 0.4 \\
\hline 17351 & 1769 & 0.9 & 0.4 \\
\hline 17366 & 1782 & 0.0 & 0.2 \\
\hline 17389 & 1811 & 0.6 & 0.3 \\
\hline 17391 & 1872 & 0.1 & 0.1 \\
\hline 17422 & 1785 & 0.1 & 0.3 \\
\hline 17435 & 1902 & 0.1 & 0.4 \\
\hline 17436 & 1790 & 0.4 & 0.3 \\
\hline 17464 & 1853 & 0.5 & 0.3 \\
\hline 17486 & 1854 & 1.0 & 0.3 \\
\hline 17497 & 1837 & 0.4 & 0.3 \\
\hline 17500 & 2249 & 0.0 & 0.4 \\
\hline 17535 & 1838 & 0.4 & 0.3 \\
\hline 17548 & 1825 & 0.7 & 0.1 \\
\hline 17548 & 2231 & 0.6 & 0.5 \\
\hline 17548 & 2293 & 0.8 & 0.2 \\
\hline 17552 & 1789 & 0.3 & 0.1 \\
\hline 17568 & 1810 & 0.6 & 0.1 \\
\hline 17605 & 1809 & 1.0 & 0.2 \\
\hline 17627 & 1841 & 0.7 & 0.3 \\
\hline 17629 & 1851 & 0.8 & 0.4 \\
\hline 17647 & 1875 & 0.2 & 0.7 \\
\hline 17703 & 1881 & 0.2 & 0.2 \\
\hline 17745 & 2161 & 0.6 & 0.1 \\
\hline 17746 & 1873 & 0.1 & 0.2 \\
\hline 17784 & 1842 & 0.0 & 0.5 \\
\hline 17790 & 1887 & 0.0 & 0.6 \\
\hline 17794 & 1906 & 0.0 & 0.4 \\
\hline 17811 & 1816 & 0.0 & 0.2 \\
\hline 17814 & 1901 & 0.8 & 0.5 \\
\hline 17825 & 1819 & 0.1 & 0.2 \\
\hline 17854 & 2230 & 0.0 & 0.7 \\
\hline 17875 & 1817 & 0.4 & 0.3 \\
\hline 17880 & 1843 & 0.1 & 0.4 \\
\hline 17880 & 1957 & 0.4 & 0.2 \\
\hline 17880 & 2194 & 0.8 & 0.6 \\
\hline 17886 & 1844 & 0.1 & 0.3 \\
\hline 17924 & 1826 & 1.0 & 0.3 \\
\hline 17973 & 1926 & 0.9 & 0.3 \\
\hline 17973 & 1942 & 1.0 & 0.1 \\
\hline 18109 & 1940 & 0.3 & 0.3 \\
\hline 18325 & 2277 & 0.2 & 0.5 \\
\hline 18457 & 2285 & 0.8 & 0.2 \\
\hline 18466 & 2270 & 0.1 & 0.2 \\
\hline 18590 & 2248 & 0.9 & 0.7 \\
\hline 18596 & 2227 & 0.1 & 0.3 \\
\hline 18647 & 2271 & 0.5 & 0.2 \\
\hline
\end{tabular}


L. Östman et al.: NTT and NOT spectroscopy of SDSS-II supernovae

Table A.3. continued.

\begin{tabular}{|c|c|c|c|}
\hline ID & SPID & Host contamination $^{a}$ & Slit loss ${ }^{l}$ \\
\hline 18697 & 2171 & 0.7 & 0.2 \\
\hline 18768 & 2135 & 0.1 & 0.5 \\
\hline 18787 & 2150 & 0.2 & 0.5 \\
\hline 18804 & 2148 & 0.2 & 0.5 \\
\hline 18903 & 2247 & 0.8 & 0.5 \\
\hline 18965 & 2279 & 0.6 & 0.5 \\
\hline 19003 & 2235 & 0.9 & 0.9 \\
\hline 19003 & 2290 & 0.9 & 0.5 \\
\hline 19008 & 2284 & 0.8 & 0.2 \\
\hline 19023 & 2236 & 0.0 & 0.4 \\
\hline 19051 & 2297 & 0.5 & 0.2 \\
\hline 19101 & 2268 & 0.1 & 0.2 \\
\hline 19149 & 2275 & 0.0 & 0.4 \\
\hline 19155 & 2252 & 0.3 & 0.9 \\
\hline 19155 & 2607 & 0.3 & 0.0 \\
\hline 19155 & 2720 & 0.8 & 0.8 \\
\hline 19221 & 2274 & 0.5 & 0.5 \\
\hline 19222 & 2299 & 0.2 & 0.2 \\
\hline 19230 & 2282 & 0.9 & 0.4 \\
\hline 19282 & 2280 & 0.1 & 0.4 \\
\hline 19323 & 2296 & 0.5 & 0.6 \\
\hline 19341 & 2298 & 0.3 & 0.1 \\
\hline 19353 & 2281 & 0.3 & 0.4 \\
\hline 19381 & 2283 & 0.1 & 0.5 \\
\hline 19899 & 2550 & 0.0 & 0.0 \\
\hline 19913 & 2585 & 0.4 & 0.1 \\
\hline 19953 & 2602 & 0.2 & 0.0 \\
\hline 19968 & 2549 & 0.1 & 0.1 \\
\hline 20039 & 2584 & 0.6 & 0.7 \\
\hline 20040 & 2612 & 0.4 & 0.8 \\
\hline 20052 & 2537 & 0.1 & 0.0 \\
\hline 20052 & 2538 & 0.2 & 0.9 \\
\hline 20088 & 2546 & 0.8 & 0.7 \\
\hline 20097 & 2587 & 0.1 & 0.5 \\
\hline 20142 & 2586 & 0.4 & 0.7 \\
\hline 20144 & 2541 & 0.2 & 1.0 \\
\hline 20227 & 2568 & 0.7 & 0.6 \\
\hline 20345 & 2567 & 0.1 & 1.0 \\
\hline 20364 & 2581 & 0.4 & 1.0 \\
\hline 20376 & 2582 & 0.8 & 0.1 \\
\hline 20388 & 2611 & 0.6 & 0.8 \\
\hline 20430 & 2543 & 0.1 & 1.0 \\
\hline 20474 & 2563 & 0.4 & 0.5 \\
\hline 20474 & 2714 & $0.6^{e}$ & 0.1 \\
\hline 20474 & 3003 & $0.7^{e}$ & 0.1 \\
\hline 20530 & 2547 & 0.9 & 0.6 \\
\hline 20530 & 2571 & 0.8 & 0.8 \\
\hline 20575 & 2540 & 0.9 & 0.1 \\
\hline 20575 & 3005 & 0.8 & 0.1 \\
\hline 20625 & 2551 & 0.4 & 0.0 \\
\hline 20625 & 2604 & 0.3 & 0.0 \\
\hline 20677 & 2536 & 0.9 & 0.7 \\
\hline 20678 & 2610 & 0.8 & 0.1 \\
\hline 20687 & 2596 & 0.9 & 0.1 \\
\hline 20687 & 2597 & 0.9 & 0.1 \\
\hline 20718 & 2577 & 0.9 & 0.4 \\
\hline 20718 & 2593 & 0.8 & 0.1 \\
\hline 20764 & 2594 & 0.7 & 0.1 \\
\hline 20834 & 2598 & 0.9 & 1.0 \\
\hline 20862 & 2600 & 0.7 & 0.1 \\
\hline 20909 & 2580 & 0.8 & 1.0 \\
\hline 20978 & 2609 & 0.2 & 1.0 \\
\hline 21006 & 2566 & 0.0 & 0.8 \\
\hline 21033 & 2565 & 0.1 & 0.7 \\
\hline 21034 & 2719 & 0.4 & 0.8 \\
\hline 21034 & 2733 & 0.7 & 0.3 \\
\hline 21042 & 2564 & 0.4 & 0.8 \\
\hline
\end{tabular}


A\&A 526, A28 (2011)

Table A.3. continued.

\begin{tabular}{cccc}
\hline \hline ID & SPID & Host contamination $^{a}$ & Slit loss $^{b}$ \\
\hline 21058 & 2579 & 0.5 & 0.8 \\
21058 & 2595 & 0.5 & 0.1 \\
21062 & 2613 & 0.3 & 0.9 \\
21064 & 2532 & 0.2 & 0.1 \\
21064 & 2533 & 0.3 & 0.5 \\
21362 & 2636 & 0.3 & 0.0 \\
21362 & 2697 & $0.3^{e}$ & 1.0 \\
21422 & 2599 & 0.1 & 0.3 \\
21502 & 2574 & 0.3 & 0.0 \\
21502 & 2575 & 0.5 & 0.1 \\
21502 & 2717 & 0.7 & 0.4 \\
21596 & 2588 & 0.8 & 0.1 \\
21596 & 2589 & 0.8 & 1.0 \\
21669 & 2591 & 0.2 & 0.7 \\
21669 & 2722 & $0.1^{e}$ & 0.3 \\
21766 & 2638 & 1.0 & 0.0 \\
21810 & 2724 & $0.1^{e}$ & 0.1 \\
21814 & 2702 & 0.9 & 0.6 \\
21839 & 2716 & $0.4^{e}$ & 0.1 \\
21861 & 2723 & $0.2^{e}$ & 0.9 \\
21898 & 2704 & $1.0^{e}$ & 0.9 \\
22182 & 2690 & $0.0^{e}$ & 0.7 \\
22284 & 2735 & $0.6^{e}$ & 0.3 \\
\hline
\end{tabular}

Notes. Both these quantities are very rough estimates and are in most cases likely overestimated.

(a) The estimated host-galaxy contamination in the observed spectra, i.e. before host-galaxy subtraction has been attempted, for wavelengths corresponding to the $g$ filter in observed frame. A superscript of " $e$ " is used when the estimation is based on an extrapolation of the photometry. (b) The estimated maximum differential slit loss due to atmospheric refraction affecting the SN light in the wavelength region $4000-8000 \AA$ (observed frame). 